\title{
SUSY meets her twin
}

\section{Andrey Katz, ${ }^{a, b}$ Alberto Mariotti, ${ }^{c}$ Stefan Pokorski, ${ }^{d}$ Diego Redigolo ${ }^{e, f}$ and Robert Ziegler ${ }^{g}$}

${ }^{a}$ Theory Division, CERN, CH-1211 Geneva 23, Switzerland

${ }^{b}$ Département de Physique Théorique and Center for Astroparticle Physics (CAP), Université de Genève,

24 quai Ansermet, CH-1211 Genève 4, Switzerland

${ }^{c}$ Theoretische Natuurkunde and IIHE/ELEM, Vrije Universiteit Brussel, and International Solvay Institutes, Pleinlaan 2, B-1050 Brussels, Belgium

${ }^{d}$ Institute of Theoretical Physics, Faculty of Physics, University of Warsaw, ul. Pasteura 5, PL-02-093 Warsaw, Poland

${ }^{e}$ Raymond and Beverly Sackler School of Physics and Astronomy, Tel-Aviv University, Tel-Aviv 69978, Israel

${ }^{f}$ Department of Particle Physics and Astrophysics, Weizmann Institute of Science, Rehovot 7610001, Israel

${ }^{g}$ Institute for Theoretical Particle Physics (TTP), Karlsruhe Institute of Technology, Engesserstraße 7, D-76128 Karlsruhe, Germany

E-mail: andrey.katz@cern.ch, Alberto.mariotti@vub.ac.be, pokorski@fuw.edu.pl, d.redigolo@gmail.com, robert.ziegler@kit.edu

ABSTRACT: We investigate the general structure of mirror symmetry breaking in the Twin Higgs scenario. We show, using the IR effective theory, that a significant gain in fine tuning can be achieved if the symmetry is broken hardly. We emphasize that weakly coupled UV completions can naturally accommodate this scenario. We analyze SUSY UV completions and present a simple Twin SUSY model with a tuning of around $10 \%$ and colored superpartners as heavy as $2 \mathrm{TeV}$. The collider signatures of general Twin SUSY models are discussed with a focus on the extended Higgs sectors.

KEYWoRds: Supersymmetry Phenomenology

ARXIV EPRINT: 1611.08615 


\section{Contents}

1 Introduction 1

2 EWSB and fine tuning in Twin Higgs models 3

2.1 Setup 3

2.2 Electroweak symmetry breaking and radiative corrections 5

2.3 Fine tuning in the low energy effective theory and beyond 8

3 SUSY UV completions

$\begin{array}{lll}3.1 & \text { Soft Twin SUSY } & 13\end{array}$

3.2 Hard Twin SUSY 21

4 Twin SUSY Higgs phenomenology $\quad 26$

$\begin{array}{lll}4.1 & \text { The Higgs sector of Twin SUSY } & 26\end{array}$

4.2 Probing the SUSY Twin Higgses 30

5 Conclusions $\quad 35$

A Renormalization Group Equations $\quad 37$

$\begin{array}{lll}\text { A.1 } & \text { Soft } \mathbb{Z}_{2} \text {-breaking model } & 37\end{array}$

$\begin{array}{lll}\text { A.2 } & \text { Hard } \mathbb{Z}_{2} \text {-breaking + bi-doublets } & 39\end{array}$

B Higgs sector spectrum $\quad 41$

B.1 The Twin Higgs as a linear sigma model 42

B.2 The SUSY Twin Higgs 43

B.3 Approximate expressions for eigenvalues and eigenvectors 47

$\begin{array}{lll}\text { B.4 Decay rates } & 48\end{array}$

\section{Introduction}

Supersymmetry (SUSY) and Compositeness are the leading new physics (NP) candidates to solve the hierarchy problem of the Standard Model (SM). However, the null results of the LHC searches for new colored particles already put these ideas under pressure. While it is plausible that one of these theories indeed solves the big hierarchy problem, it is not easy to see how one can avoid the little hierarchy problem, i.e. the mismatch between the electroweak (EW) scale and the scale of top-partners. It is certainly possible that this residual hierarchy is not resolved in nature, and naturalness might not be the only criterion for physics beyond the SM (BSM). Nevertheless, it is interesting to explore alternatives that can solve the little hierarchy problem and circumvent the LHC bounds.

The Neutral Naturalness (NN) paradigm is an attractive idea in this context. It provides a scenario where the top partners are charged under an $\mathrm{SU}(3)$ group that is 
different from the SM color group. As a consequence they can be as light as required by naturalness while evading LHC bounds, because of reduced production cross sections compared to the usual colored top-partners. Known realizations of NN include models where the top partners are fermions (twin tops [1]) and models where they are bosons (e.g. the folded stops in the Folded SUSY model [2]).

Our work focuses on the Twin Higgs scenario, in which the SM Higgs is a pseudoGoldstone boson (PGB) of an accidental SU(4) symmetry emerging from a $\mathbb{Z}_{2}$ symmetry that exchanges the SM with a mirror SM. ${ }^{1}$ Generalizations of the Twin Higgs mechanism that involves more general discrete symmetries [7-9] also share some basic features with the simplest model.

In spite of its simplicity, the Twin Higgs introduces important theoretical challenges that we try to address in this paper. The first one has to do with the actual gain in fine tuning (FT) of this setup. Even though the Twin Higgs was originally suggested to ameliorate the little hierarchy problem, all its known realizations feature an upper bound on their parametric gain in FT with respect to colored naturalness models. This gain cannot be more than $\lambda / \lambda_{\mathrm{SM}}$, where $\lambda$ is a perturbative SU(4)-symmetric Higgs quartic coupling and $\lambda_{\mathrm{SM}}$ is the quartic coupling of the SM potential (see for example ref. [10] for a discussion of this bound). A similar bound has been observed in double protection models, not necessarily related to the NN proposal [11-17].

The second theoretical challenge concerns the fact that any NN construction only solves the little hierarchy problem. Above the scale of SU(4) symmetry breaking the Twin Higgs models should be embedded into a framework that solves the big hierarchy problem. Embeddings in SUSY [18-20] and in Composite Higgs [4-6] feature the parametric gain in FT we described above. In SUSY UV completions, $\lambda / \lambda_{\mathrm{SM}}$ is severely constrained by the perturbativity bound on $\lambda$ [20]. This bound can be relaxed in Composite Twin Higgs scenarios. The latter, however, are far from achieving $\lambda \sim 4 \pi[10,21]$.

The mirror symmetry in the Higgs potential can be softly-broken by a mass term and/or hardly-broken by a quartic coupling. In this paper we give a systematic overview of these breaking patterns and discuss their relations to the FT. ${ }^{2}$ The main result of our analysis is that the above mentioned parametric bound on the FT improvement is not a generic feature of the Twin Higgs construction but rather an artifact of the soft mirror-symmetry breaking. This bound can be circumvented if the breaking is dominantly hard, which is however difficult to realize in composite UV completions of the Twin Higgs and therefore has been mostly disregarded in the literature (notable exceptions are the models presented in $[19,26])$.

We present viable UV completions of the Twin Higgs in Supersymmetry, where both hard and soft breaking of the mirror symmetry can be easily achieved, and study their LHC phenomenology. We show that the FT gain of hard $\mathbb{Z}_{2}$-breaking models is substantial and only limited by the Higgs mass constraint.

\footnotetext{
${ }^{1}$ Having in mind perturbative UV completions of the Twin Higgs, the SU(4) accidental symmetry is automatically enhanced to $\mathrm{SO}(8)$ and we do not need to distinguish between the two symmetry groups. We refer to [3-6] for a careful discussion of the importance of this difference in strongly coupled UV completions.

${ }^{2} \mathrm{~A}$ viable spontaneous $\mathbb{Z}_{2}$-breaking necessarily requires extra non-decoupled Higgses and will not be discussed in this paper, see however refs. [22-25].
} 
Our paper is structured as follows. We review the Twin Higgs mechanism with an emphasis on its symmetries and the FT gain in section 2, where we work exclusively in the Twin Higgs IR effective theory and do not ask any questions about possible UV completions. We reproduce the bound on the FT for soft $\mathbb{Z}_{2}$-breaking and show how this bound can be relaxed by adding hard $\mathbb{Z}_{2}$-breaking. We analyze in detail the parameter space of the IR effective theory, emphasize the role of the SM-like Higgs mass constraint, and discuss how this requirement determines the final gain in FT of hard $\mathbb{Z}_{2}$-breaking vs soft $\mathbb{Z}_{2}$-breaking.

In section 3 we discuss SUSY UV completions of the Twin Higgs. First, we review the SUSY Twin Higgs with soft $\mathbb{Z}_{2}$-breaking focusing on the simplest scenario of ref. [20]. We explore the parameter space of this model showing that its FT gain scales as $\lambda / \lambda_{\mathrm{SM}}$ and the effective SU(4)-invariant quartic $\lambda$ is far from being maximized. Second, we construct SUSY Twin Higgs models with hard $\mathbb{Z}_{2}$-breaking. The minimal model fails to decouple the colored states due the Higgs mass constraint. We show that in next to minimal realizations we can comply with such a constraint, decouple the colored states and achieve a FT of around $10 \%$, a factor of about 5 better than in the soft $\mathbb{Z}_{2}$-breaking scenario.

In section 4 we present a phenomenological analysis restricted to the extended Higgs sectors of Twin SUSY theories. We leave for future investigations an analysis including the full matter sector, which can lead to many other interesting signatures both at the LHC and in cosmological observables (see e.g. refs. [27-31] for further LHC studies and [3237] for the exploration of viable cosmological scenarios). The best probes of Twin SUSY constructions are certainly direct searches of the Twin Higgs, which looks very much like a singlet mixing with the SM Higgs [38-42] and for this reason can be hunted for in direct searches of a resonance decaying into di-bosons. Such searches are always better suited for weakly coupled UV completions of the Twin Higgs rather than indirect searches from Higgs coupling measurements. We also find that if the twin Higgs becomes too heavy to be abundantly produced at the LHC, the MSSM-like Higgses become sensibly lighter than their mirror states. In this case we can hunt for them in direct searches for MSSM Higgses in the low $\tan \beta$ region [43], via their indirect effects on SM Higgs couplings and on the $b \rightarrow s \gamma$ transition rate [44].

Section 5 is devoted to our conclusions and future directions. The technical details, such as RGE formulae, detailed calculations of the Higgs mass spectrum and branching ratios are relegated to appendices.

\section{EWSB and fine tuning in Twin Higgs models}

In this section we discuss the general structure of the Twin Higgs potential, and study the fine tuning associated to soft and hard $\mathbb{Z}_{2}$-breaking. We suggest that the hard breaking can lead to a substantial improvement in FT.

\subsection{Setup}

The basic idea of the Twin Higgs mechanism is that the SM-like Higgs is light compared to the new physics scale, because it is a pseudo-Goldstone boson (PGB) of an approximate 
global $\mathrm{SU}(4)$ symmetry, spontaneously broken down to $\mathrm{SU}(3)$. This $\mathrm{SU}(4)$ is merely an accidental symmetry that holds at one-loop at the level of quadratic terms, as a result of a $\mathbb{Z}_{2}$ mirror symmetry between the SM and the "twin" sector. As we will see later, this $\mathbb{Z}_{2}$ has to be broken explicitly in the Higgs potential, in order to allow for a realistic Higgs sector. By construction the Twin Higgs model is an effective theory that can resolve the hierarchy problem only up to a scale of around $\sim 5 \mathrm{TeV}$. Above this scale it should be UV-completed by a theory which solves the big hierarchy problem.

The gauge group of the Twin Higgs is extended to two copies of the SM, which we denote by $G_{A}$ and $G_{B}$, where here and in the following $A$ refers to the visible sector and $B$ to the twin sector. The Higgs sector of the original Twin Higgs model [1] consists of two copies of the SM Higgs potential, with two Higgs doublets $H_{A}$ and $H_{B}$ transforming under $G_{A}$ and $G_{B}$ respectively. The full matter content of the SM is also doubled in the $A$ and $B$ sector. However, for the purpose of analyzing the FT, we focus on the top quarks and their twin partners that have the largest couplings to the Higgs sector, in the spirit of ref. [27]. Although the contribution of the gauge sector to the little hierarchy problem is not negligible numerically, we do not address it explicitly, since the discussion closely follows the one of the top sector. We also assume for simplicity a perfect mirror $\mathbb{Z}_{2}$ symmetry between the visible and the twin sector, which we break explicitly only in the Higgs sector. In agreement with the original model we imagine that the low energy effective theory has no light states that carry both visible and mirror quantum numbers. ${ }^{3}$

Let us now write down the most general renormalizable Higgs potential for the visible and the twin Higgs:

$$
V=\lambda\left(\left|H_{A}\right|^{2}+\left|H_{B}\right|^{2}\right)^{2}+m^{2}\left(\left|H_{A}\right|^{2}+\left|H_{B}\right|^{2}\right)+\kappa\left(\left|H_{A}\right|^{4}+\left|H_{B}\right|^{4}\right)+\tilde{\mu}^{2}\left|H_{A}\right|^{2}+\rho\left|H_{A}\right|^{4} .
$$

Other terms one might be tempted to write down lead to an equivalent potential up to redefinitions of the existing couplings. We now analyze this potential dividing all the terms into three different categories: $\mathrm{SU}(4)$-preserving, SU(4)-breaking but $\mathbb{Z}_{2}$-preserving, and $\mathbb{Z}_{2}$-breaking.

1. SU(4)-preserving terms. These are the first two in the potential, to $\lambda$ and $m^{2}$. If $m^{2}<0$ and $\lambda>0$, the global $\mathrm{SU}(4)$ symmetry is spontaneously broken down to $\mathrm{SU}(3)$. We denote the scale of the $\mathrm{SU}(4)$ breaking by $f$, and consequently $f^{2} \equiv$ $v_{A}^{2}+v_{B}^{2}$. If we disregard the rest of the potential, after the breaking of the $\mathrm{SU}(4)$ global symmetry down to $\mathrm{SU}(3)$ we get one real scalar (the so-called radial mode or the "twin Higgs") with mass $m_{h_{T}}=2 \sqrt{\lambda} f$ and seven Goldstone bosons. After we gauge the $\mathrm{SU}(2) \times \mathrm{SU}(2)$ subgroup of the $\mathrm{SU}(4)$, and identify it with the visible and dark ${ }^{4} \mathrm{EW}$ gauge groups, 6 of the Goldstone bosons are "eaten" by the visible and the mirror $W$ and $Z$-bosons, while the remaining one is identified with the SM Higgs boson.

\footnotetext{
${ }^{3}$ States carrying both $G_{A}$ and $G_{B}$ quantum numbers can be present in other implementations of the Twin Higgs mechanism, for example if $\mathrm{SU}(3)_{A} \times \mathrm{SU}(3)_{B}$ is embedded in $\mathrm{SU}(6)$ [1, 4]. See ref. [31] for a discussion of the phenomenology of these states.

${ }^{4}$ We use the words dark, twin, mirror interchangeably in this paper.
} 
2. $\mathrm{SU}(4)$-breaking but $\mathbb{Z}_{2}$-preserving. This is the term proportional to $\kappa$. If this coefficient is smaller than zero, the mirror symmetry is spontaneously broken in the vacuum, and only one of the Higgses, either $H_{A}$ or $H_{B}$, gets a VEV. This possibility does not lead to a viable phenomenology in our setup and we will not discuss it further (see however ref. [22]). Instead, if $\kappa$ is larger than zero, the $\mathbb{Z}_{2}$ symmetry is preserved by the vacuum, and $H_{A}$ and $H_{B}$ get equal VEVs $v_{A}=v_{B}=f / \sqrt{2}$. We further consider $0<\kappa \ll \lambda$, such that the $\mathrm{SU}(4)$-breaking term gives a sub-leading contribution to the mass of the radial mode, but generates a small SM-like Higgs mass $m_{h}=\sqrt{2 \kappa} f$. The hierarchy between the two quartics in the potential is technically natural and so is the hierarchy between the radial mode and the SM Higgs, because the latter is a PGB in the limit $\kappa \ll \lambda$. The main problem with the model at the present stage is the unbroken $\mathbb{Z}_{2}$ symmetry which implies that the SM Higgs is an equal superposition of the visible and the mirror Higgs and it couples with equal strength to the mirror and the visible gauge bosons and matter. The couplings of the SM-like Higgs to the visible gauge bosons and fermions would be then reduced by a factor $1 / \sqrt{2}$. This scenario is excluded both by LEP EWPM and the LHC Higgs coupling measurements. Therefore we must include also explicit $\mathbb{Z}_{2}$-breaking terms.

3. $\mathbb{Z}_{2}$-breaking terms. There are two ways to break the mirror symmetry within a renormalizable theory: via a relevant operator proportional to $\tilde{\mu}$ in eq. (2.1), or via a marginal one - the $\rho$ term. We define $\tilde{\mu}^{2} \equiv \sigma f^{2}$ and work with the dimensionless parameters $\sigma$ and $\rho$, which are responsible for the Higgs VEV misalignment and the mixing angle between the SM-like Higgs and the twin Higgs. Maximal mixing is already excluded and the largest possible misalignment allowed by data translates into the bound $f / v \gtrsim 2.3$ or $f \gtrsim 400 \mathrm{GeV}$ (see for example ref. [38]).

\subsection{Electroweak symmetry breaking and radiative corrections}

In order to analyze EWSB and the Higgs mass in the Twin Higgs scenario it is instructive to integrate out the heavy radial mode and switch to an effective Higgs theory, where we can write the Higgs fields in a non-linear realization as

$$
H_{A}=f \sin \frac{\phi}{\sqrt{2} f}, \quad H_{B}=f \cos \frac{\phi}{\sqrt{2} f} .
$$

Hereafter we identify $\phi$ with the SM-like Higgs. It is straightforward to plug these expressions into eq. (2.1) and obtain the effective SM Higgs potential in the low energy effective theory. Minimization of this potential with respect to $\phi$ yields the following expressions for the VEV and the mass of the SM-like Higgs: ${ }^{5}$

$$
\begin{aligned}
& \frac{2 v^{2}}{f^{2}}=\frac{2 \kappa-\sigma}{2 \kappa+\rho}, \\
& m_{h}^{2}=4 v^{2}(2 \kappa+\rho)\left(1-\frac{v^{2}}{f^{2}}\right)=2 f^{2}(2 \kappa-\sigma)\left(1-\frac{v^{2}}{f^{2}}\right) .
\end{aligned}
$$

\footnotetext{
${ }^{5}$ The same expressions can alternatively be obtained at the level of the linear sigma model (2.1) by solving the EWSB conditions and expanding them at leading order in $\kappa, \sigma \ll \lambda$. We refer to appendix B.1 for a discussion of the sub-leading corrections in this expansion.
} 
Most of previous work on Twin Higgs has concentrated on soft $\mathbb{Z}_{2}$-breaking, i.e $\sigma \gg \rho$. In this paper we go further and consider hard $\mathbb{Z}_{2}$-breaking, i.e. the presence of a tree-level $\rho$, such that $\rho \gg \sigma$. A hard breaking of the mirror symmetry was already introduced in some specific Twin Higgs models (see for example refs. [19, 26]). Note that, in models where $\mathbb{Z}_{2}$-breaking effects are generated by loops of the matter/gauge sector, $\rho$ is unavoidably generated. In that case, however, it is typically smaller than $\sigma \cdot{ }^{6}$ Here for the first time we attempt to provide a systematic understanding of the breaking of $\mathbb{Z}_{2}$ mirror symmetry in the Higgs sector from the EFT point of view.

As a guideline for model building, it is very helpful to visualize the parameter space of the Twin Higgs model when both soft and hard $\mathbb{Z}_{2}$-breaking terms are present. In order to do this we solve the equations for the measured values of the Higgs mass (2.4) and the VEV (2.3) and remain with only two free parameters, ${ }^{7}$ which we choose to be the $\mathbb{Z}_{2}$-breaking ones, namely $\sigma$ and $\rho$. The values of the $\mathbb{Z}_{2}$-preserving quartic $\kappa$ and of the $\mathrm{SU}(4)$-breaking scale $f$ are then fixed at each point in the $(\sigma, \rho)$ plane. We show the contours of $\kappa$ and the region that satisfy the constraint $f / v>2.3$ in figure 1 . The first striking message of this figure is that, given the values of the Higgs boson mass and the electroweak VEV, the acceptable values of the $\mathbb{Z}_{2}$-odd parameters are confined to a narrow band in the $\mathbb{Z}_{2}$-breaking parameter space. It is also instructive to consider the two limiting cases of dominant soft and dominant hard $\mathbb{Z}_{2}$-breaking. The corresponding regions in the parameter space are marked in figure 1 with red and green stars, respectively. The values of $\kappa$ in both regions can also be inferred from the figure: in the hard $\mathbb{Z}_{2}$-breaking region $\kappa$ is forced to be roughly a factor of 5 smaller than in the soft $\mathbb{Z}_{2}$-breaking region as a consequence of the Higgs mass constraint.

Now we are ready to include the radiative corrections to the various parameters. These are coming from top loops and EW gauge boson loops (we discuss only the former in detail) but also from Higgs quartic loops, because the hard $\mathbb{Z}_{2}$-breaking quartic $\rho$ reintroduces for $\sigma$ a quadratic sensitivity to the mass threshold at which $\mathbb{Z}_{2}$-breaking is generated.

For the radiative top corrections, we should a priori consider the contributions of both visible and mirror tops and assume that the numerical value of the top-Higgs coupling in the visible and the twin sector can be different from one another. However, as was already pointed out in ref. [27], the top Yukawas in the visible and the hidden sector should agree to a level of better than $1 \%$ in order to avoid an unacceptable FT. To simplify our discussion we enforce an exact $\mathbb{Z}_{2}$ symmetry between the top sectors (and the gauge sectors also).

The dominant radiative corrections to the dimensionless parameters read

$$
\begin{aligned}
\Delta \kappa & =\frac{3 y_{t}^{4}}{16 \pi^{2}} \log \frac{\Lambda_{t}^{2}}{m_{t_{B}}^{2}}+\frac{3 \lambda \rho}{32 \pi^{2}}\left(\log \frac{\Lambda_{\rho}^{2}}{m_{h_{T}}^{2}}+\log \frac{\Lambda_{\rho}^{2}}{m_{h}^{2}}\right), \\
\Delta \rho & =\frac{3 y_{t}^{4}}{16 \pi^{2}} \log \frac{f^{2}}{v^{2}},
\end{aligned}
$$

\footnotetext{
${ }^{6}$ A notable example are models where the "twin" hypercharge $\mathrm{U}(1)_{B}$ is ungauged $[27]$ and $\mathbb{Z}_{2}$-breaking effects are generated by $\mathrm{U}(1)_{A}$ gauge loops. In this case $\sigma \sim g_{1}^{2} / 16 \pi^{2}$ and $\rho \sim g_{1}^{4} / 16 \pi^{2}$ and the latter can safely be neglected.

${ }^{7}$ We use $m_{h}=125 \mathrm{GeV}$ and $v=174 \mathrm{GeV}$ in all our calculations. Here we allow for a $\pm 5 \mathrm{GeV}$ Higgs mass shift because we do not take into account higher order corrections.
} 


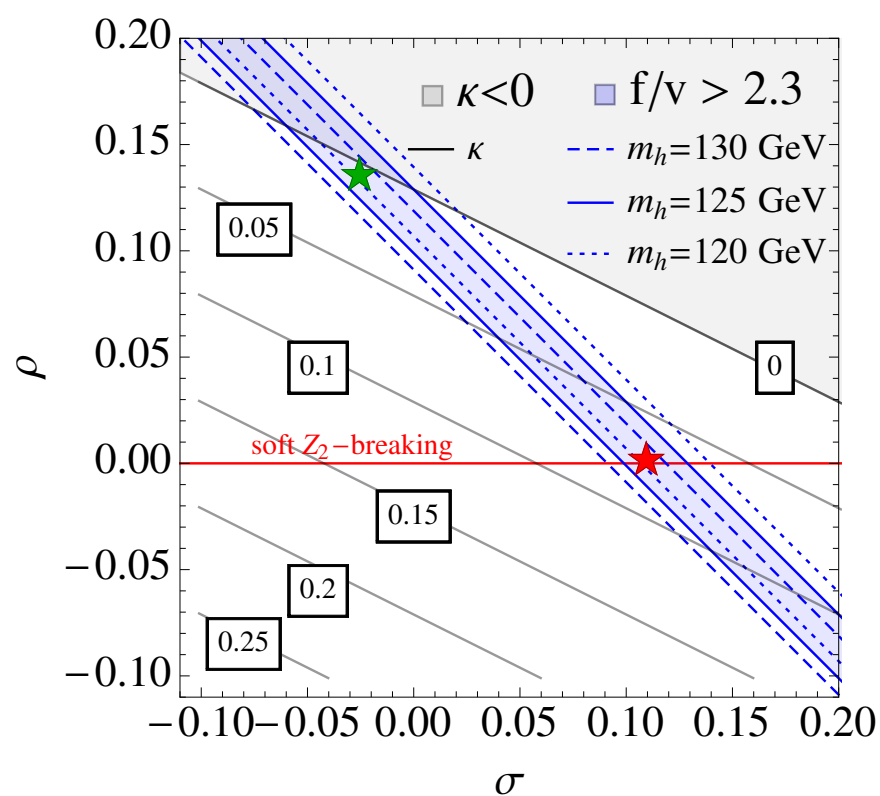

Figure 1. The blue region inside the solid blue lines corresponds to the allowed parameter space with $f / v>2.3$ and $m_{h}=125 \mathrm{GeV}$. The dashed (dotted) blue lines shows how the allowed region shifts if one assumes $m_{h}=120 \mathrm{GeV}\left(m_{h}=130 \mathrm{GeV}\right)$. The black lines correspond to the values of $\kappa$. The non-viable region with negative $\kappa$ is shaded in grey. The green/red star indicates the region of hard/soft breaking.

$$
\Delta \sigma=\frac{3 \rho}{16 \pi^{2}}\left(\epsilon \frac{\Lambda_{\rho}^{2}}{f^{2}}+2 \lambda \log \frac{\Lambda_{\rho}^{2}}{m_{h}^{2}}\right),
$$

where we kept frozen the Higgs dependence in the logarithms and expanded to first order in $\kappa$ and $v / f$. The running top Yukawa coupling, $y_{t}$, is evaluated at the UV cutoff scale $\Lambda_{t}$, fixing the scheme dependence of our one-loop computation.

Several clarifications are in order. First, we introduced in these expressions two different mass thresholds: $\Lambda_{t}$ and $\Lambda_{\rho}$, cutting-off the top loops and the Higgs loops respectively. Loosely speaking, in a UV complete natural theory $\Lambda_{t}$ will be identified with the mass scale of new colored states, while the states associated with $\Lambda_{\rho}$ can be complete SM singlets. While in strongly coupled UV completions it is hard to imagine a wide separation between $\Lambda_{t}$ and $\Lambda_{\rho}$, in weakly coupled UV completions (e.g. SUSY) there could be appreciable differences between the two. Therefore, we keep track of these scales separately and we will see in the concrete SUSY models of section 3 that $\Lambda_{t}$ and $\Lambda_{\rho}$ correspond to different SUSY mass thresholds. Of course we should keep in mind that these scales cannot be arbitrarily separated from one another, because of higher-order quantum corrections.

Second, in eq. (2.7) we introduced a new parameter $\epsilon$. This parameter stand for the sign of the UV mass threshold corrections and a priori $\epsilon= \pm 1$. Since one cannot calculate the sign of these radiative corrections within the IR effective theory, we remain agnostic and consider both positive and negative threshold corrections. As we will see in the next section, within a full UV complete theory this sign is determined unambiguously. 


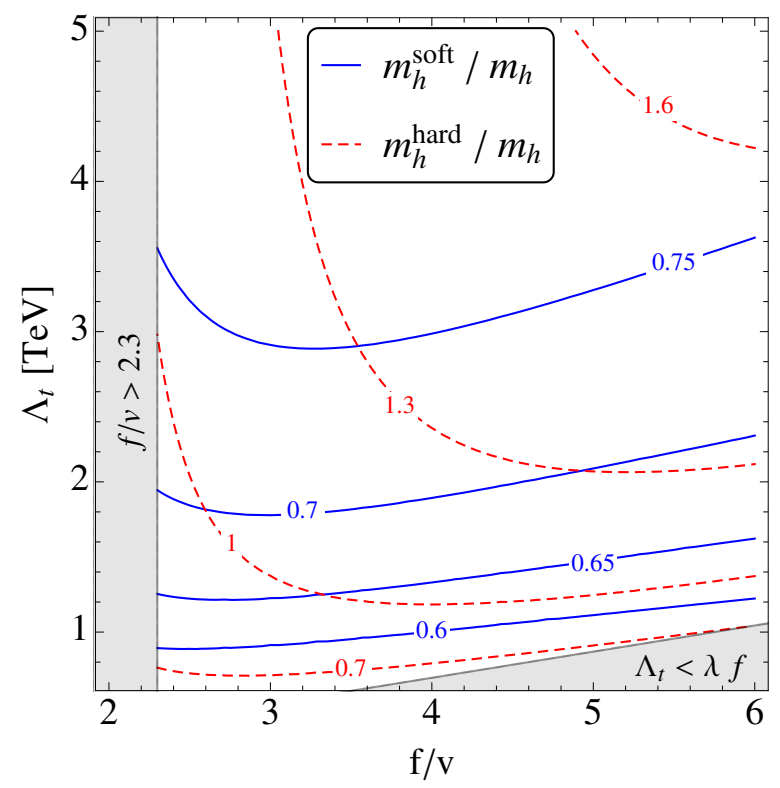

Figure 2. The blue contours indicates the irreducible contributions to the Higgs mass in the pure soft breaking scenario $\left(\rho_{0}=0\right)$, coming from top loops normalized to $m_{h}=125 \mathrm{GeV}$. The red dashed contours indicate the irreducible contributions to the Higgs mass in the pure hard breaking case $\left(\sigma_{0}=0\right)$, where we fix $\lambda=1, \Lambda_{\rho}=1 \mathrm{TeV}$ and $\epsilon=+1$ in eqs. (2.5) and (2.7). The grey shaded region at the left edge with $f<2.3 v$ is excluded by Higgs coupling measurements and the grey region at the bottom right of the plot has $\Lambda_{t}<\lambda f$.

Having at hand all the radiative contributions to $\rho, \kappa$ and $\sigma$, we can estimate how big are the radiative contributions to the Higgs mass. We concentrate on two extreme cases: pure soft breaking, defined as $\rho_{0}=0$, and pure hard breaking, defined as $\sigma_{0}=0$. From eq. (2.4) we can infer that the radiative contributions to the Higgs mass squared, up to $\mathcal{O}\left(v^{2} / f^{2}\right)$ corrections, are given by $\Delta m_{h}^{2}=4 v^{2}(2 \Delta \kappa+\Delta \rho)$. We show the size of these corrections in figure 2. The first important conclusion that we draw from this figure is that while radiative corrections contribute at least $\sim 60 \%$ of the Higgs mass for pure soft $\mathbb{Z}_{2}$-breaking, the radiative corrections typically overshoot the Higgs mass for pure hard $\mathbb{Z}_{2^{-}}$ breaking, though not by orders of magnitude. In what follows we will see that in the hard breaking case the FT $\Delta_{v / f}$ can be ameliorated compared to the soft case at the price of adjusting the Higgs mass (with either a large $\Lambda_{\rho}$ or a negative tree-level contribution to $\kappa$ ).

\subsection{Fine tuning in the low energy effective theory and beyond}

We are now ready to discuss the FT quantitatively. For illustration purposes let us start from the pure soft case, where the IR FT is well-known to scale as $\sim f^{2} / v^{2}$. More precisely, quantifying the fine-tuning à la Barbieri-Giudice [45], one gets

$$
\Delta_{v / f}^{\mathrm{soft}} \equiv\left|\frac{\partial \log v^{2}}{\partial \log \sigma}\right|=\frac{f^{2}-2 v^{2}}{2 v^{2}} .
$$

This is only a part of the total fine-tuning that one can estimate in the IR effective theory. On top of that one should also consider the fine-tuning of the scale $f$, which one can think 
of as the inverse Fermi constant of the spontaneously broken SU(4), with respect to the top cutoff scale $\Lambda_{t}$. Strictly speaking this fine-tuning should be computed in the full UV theory, but one can get a reasonable estimate by analyzing the threshold corrections to the scale $f$ in the effective theory. These radiative corrections are given by

$$
\Delta f^{2}=\frac{1}{32 \pi^{2}}\left(\frac{3 y_{t}^{2}}{\lambda} \Lambda_{t}^{2}-5 \Lambda_{\lambda}^{2}\right)
$$

We will see in the next section how this expression reproduces the dominant RGE effects of the UV theory, once the "cut-off" scales are identified with physical mass thresholds. A priori the sensitivities to the thresholds $\Lambda_{t}$ and $\Lambda_{\lambda}$ are equally dangerous and, unlike in the SM, it is not clear that the dominant sensitivity comes from the tops rather than the Higgses themselves. However, at the threshold $\Lambda_{\lambda}$ we expect to find colorless particles (singlet scalars and singlinos), which are weakly constrained by the LHC. Instead, one expects the colored top partners at the scale $\Lambda_{t}$, which are more tightly bounded from direct searches. Therefore we will assume that $\Lambda_{t}>\Lambda_{\lambda}$ and will mostly worry about the top threshold as main source of the fine-tuning.

From eq. (2.9) we can estimate the fine-tuning $\Delta_{f / \Lambda}$ of the scale $f^{2}$ with respect to the scale $\Lambda_{t}$ as: ${ }^{8}$

$$
\Delta_{f / \Lambda_{t}}^{\mathrm{soft}} \equiv\left|\frac{\partial \log f^{2}}{\partial \log \Lambda_{t}^{2}}\right|=\frac{3 y_{t}^{2}}{32 \pi^{2} \lambda} \frac{\Lambda_{t}^{2}}{f^{2}} .
$$

What is now the total fine-tuning of the soft Twin Higgs model? It is tempting to say that $\Delta^{\text {soft }}=\Delta_{v / f}^{\text {soft }} \times \Delta_{f / \Lambda_{t}}^{\text {soft }}$. From the point of view of the effective field theory this factorization is definitely correct and we can estimate the total fine-tuning as

$$
\Delta^{\mathrm{soft}} \approx \frac{3 y_{t}^{2}}{32 \pi^{2} \lambda}\left(\frac{\Lambda_{t}^{2}}{2 v^{2}}\right)
$$

We will see later that this result is an excellent approximation also well beyond the effective IR theory.

Interestingly, eq. (2.11) reproduces the familiar expression for the fine-tuning in common SUSY models up to the crucial change $\lambda \leftrightarrow \lambda_{\mathrm{SM}}$, where the effective SM quartic is defined via $m_{h}^{2}=4 \lambda_{\mathrm{SM}} v^{2}$, such that $\lambda_{\mathrm{SM}} \approx 0.13$ (as we will see later we can identify $\Lambda_{t}$ with the average stop soft mass $M_{s}$ times a log encoding the dependence on the high SUSY scale). We conclude that the fine-tuning in the Twin Higgs with a softly broken $\mathbb{Z}_{2}$ can be reduced with respect to the garden-variety SUSY by a factor $\lambda_{\mathrm{SM}} / \lambda$ at best, in agreement with the results of ref. [20] and with later claims about the generic nature of the fine-tuning in Twin Higgs models (see e.g. ref. [10]). This parametric structure of the fine-tuning measure is characteristic of so-called double-protection models [11-17], in which the Higgs is a PGB, and the scale of the spontaneous global symmetry breaking is protected by SUSY.

\footnotetext{
${ }^{8}$ One should be careful at this point. $\Lambda_{t}$ "cut-off" is not a parameter of the full UV theory and therefore one technically need a full UV theory to render this calculation reliable. However, we expect this calculation to be a good estimate of the fine tuning after the cut-off is properly mapped onto the parameters of the full theory.
} 
Therefore, the fine-tuning gain is at best moderate in weakly coupled theories where perturbativity implies that $\lambda$ cannot exceed $\sim 1-1.5$. In practice, the gain is even more modest, because getting such high values of $\lambda$ is not easy in full SUSY UV complete models, where $\lambda$ is a function of other parameters of the underlying theory. This difficulty has motivated model building in the direction of strongly-coupled Twin Higgs UV completions $[5,6]$, with the hope to saturate the parametric gain by taking $\lambda \approx 4 \pi$. This kind of constructions have their own problems, most notably the large number of states below the cut-off scale and the tensions with EW precision measurements, which again give an upper bound on the value of $\lambda[10,21]$.

What happens if we consider pure hard $\mathbb{Z}_{2}$ breaking, i.e. $\sigma_{0}=0$ ? In this case $\sigma$ is entirely given by the radiative corrections in eq. (2.7). In this case the fine-tuning in the IR effective theory is given by the logarithmic variation of $v^{2}$ with respect to the parameter $\rho$ and reads

$$
\Delta_{v / f}^{\mathrm{hard}}=\frac{f^{2}-2 v^{2}}{2 v^{2}} \times F\left(v, f ; \Lambda_{\rho}\right),
$$

where we eliminated $\rho$ using the EWSB condition in eq. (2.3) and we dropped the $\log \Lambda_{\rho}$ contributions to $\sigma$ because they are largely subdominant to the quadratic contributions in most portions of the parameter space (but not all). Here we have introduced for further convenience

$$
F\left(v, f ; \Lambda_{\rho}\right) \equiv \frac{3 \epsilon \Lambda_{\rho}^{2}+32 \pi^{2} v^{2}}{3 \epsilon \Lambda_{\rho}^{2}+16 \pi^{2} f^{2}} .
$$

Interestingly the first piece in (2.12) is precisely the fine-tuning in the pure soft breaking scenario, but in the hard breaking case it is multiplied by a function $F\left(v, f ; \Lambda_{\rho}\right)$. We illustrate the behavior of this function in figure $3 .{ }^{9}$ This function is always smaller than 1 and in the limit $3 \Lambda_{\rho}^{2} \ll 32 \pi^{2} v^{2}$ reduces to $2 v^{2} / f^{2}$, while in the limit $3 \Lambda_{\rho}^{2} \gg 16 \pi^{2} f^{2}$ it saturates the upper bound of 1 . If we assume that the fine-tuning of the scale $f^{2}$ with respect to the top cutoff scale is not different from the soft case and the factorization works, this tells us (at least naively) that the reduction of the fine-tuning with respect to common SUSY scenarios should be $\left(\lambda_{\mathrm{SM}} / \lambda\right) \times F\left(v, f ; \Lambda_{\rho}\right)$, which can be a significant improvement compared to the Twin Higgs with softly broken $\mathbb{Z}_{2}$.

Alternatively one can understand the parametric dependence of the fine-tuning measure in the hard breaking scenario by fixing the cut-off scale $\Lambda_{\rho}$ using the EWSB condition in eq. (2.3), so that the fine-tuning in eq. (2.12) can be rewritten as

$$
\Delta_{v / f}^{\mathrm{hard}}=\frac{f^{2}-2 v^{2}}{2 v^{2}} \times \frac{2 \kappa}{2 \kappa+\rho}=\frac{f^{2}-2 v^{2}}{2 v^{2}} \times \frac{8 \kappa v^{2}}{m_{h}^{2}}\left(1-\frac{v^{2}}{f^{2}}\right) .
$$

This expression makes manifest that the gain in fine-tuning of the hard-breaking scenario with respect to the soft breaking one is roughly proportional to the relative size of quartic couplings $\kappa$ and $\rho$. In the second equality we have used that the combination of the two quartics in the denominator is fixed by the Higgs mass constraint (2.4), so that the gain in

\footnotetext{
${ }^{9}$ Note that on all our plots, including this figure, we single out the region where the mass thresholds are below $\lambda f$, i.e. roughly the twin Higgs mass, because we do not expect our low energy EFT to be valid there.
} 


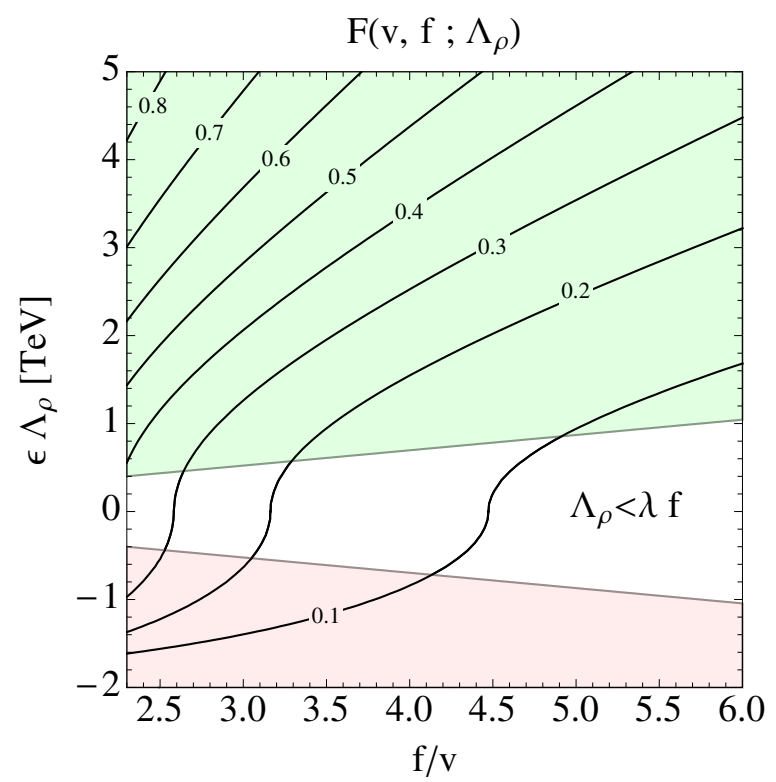

Figure 3. Contours of $F\left(v, f ; \Lambda_{\rho}\right)$ as it is defined in eq. (2.13). The two different colors (light green and light pink) correspond to the two different signs of the one-loop threshold in eq. (2.7) $(\epsilon=+1$ and $\epsilon=-1$ ). In the white cone $\Lambda_{\rho}<\lambda f$ with $\lambda=1$.

fine-tuning depends just on the value of $\kappa$, which has to be a small (see figure 1), in agreement with our requirement to minimize the fine-tuning. Clearly $\kappa$ cannot be arbitrarily small and its natural value is indicated by eq. (2.5), numerically $\mathcal{O}\left(10^{-2} \ldots 10^{-3}\right)$. As we see in figure 2 this slightly overshoots the Higgs mass, but the overshooting is $\mathcal{O}(1)$ rather than order of magnitude, therefore the fine-tuning associated with adjusting the value of $\kappa$ is also merely of order $\mathcal{O}(1)$.

In figure 4 we show the improvement in the fine-tuning of two different hard-breaking scenarios. Both these scenarios will have direct analogs in the SUSY UV completions to be discussed in section 3.2. On the left panel we consider a purely hard $\mathbb{Z}_{2}$ breaking with $\sigma_{0}=0$ and a small $\kappa_{0}$ that we take $10^{-3}$ having in mind its typical value in SUSY UV completions at small $\tan \beta$. The value of $\epsilon \times \Lambda_{\rho}$ is indicated by the black dashed isolines on the left panel of figure 4. Since $\kappa_{0}$ is fixed, this quantity is determined in order to get the measured Higgs mass value $m_{h}=125 \mathrm{GeV}$. Interestingly, in the light blue region of figure 4 where the threshold correction proportional to $\Lambda_{\rho}$ is negative (i.e. for $\epsilon=-1$ ), the Higgs mass induces a strong upper bound on $\Lambda_{t}$. In the opposite case where the threshold correction is positive, one can always adjust the Higgs mass in eq. (2.4) via a large radiatively induced $\sigma$, increasing $\Lambda_{\rho}$. Of course this comes at the price of reducing the gain in fine-tuning of the hard-breaking model, as it is shown by the blue isolines on the left panel of figure 4 .

On the right panel of figure 4 we consider a purely hard $\mathbb{Z}_{2}$-breaking case with $\sigma_{0}=0$, $\Lambda_{\rho}=1 \mathrm{TeV}$ and we fix $\epsilon=-1$ (i.e. the sign of the threshold correction in eq. (2.7)). If we require $\kappa_{0}$ to compensate for the overshooting of the Higgs mass, than a negative $\kappa_{0}$ is needed in order to avoid an upper bound on the scale of the colored states $\Lambda_{t} \lesssim 1 \mathrm{TeV}$. 

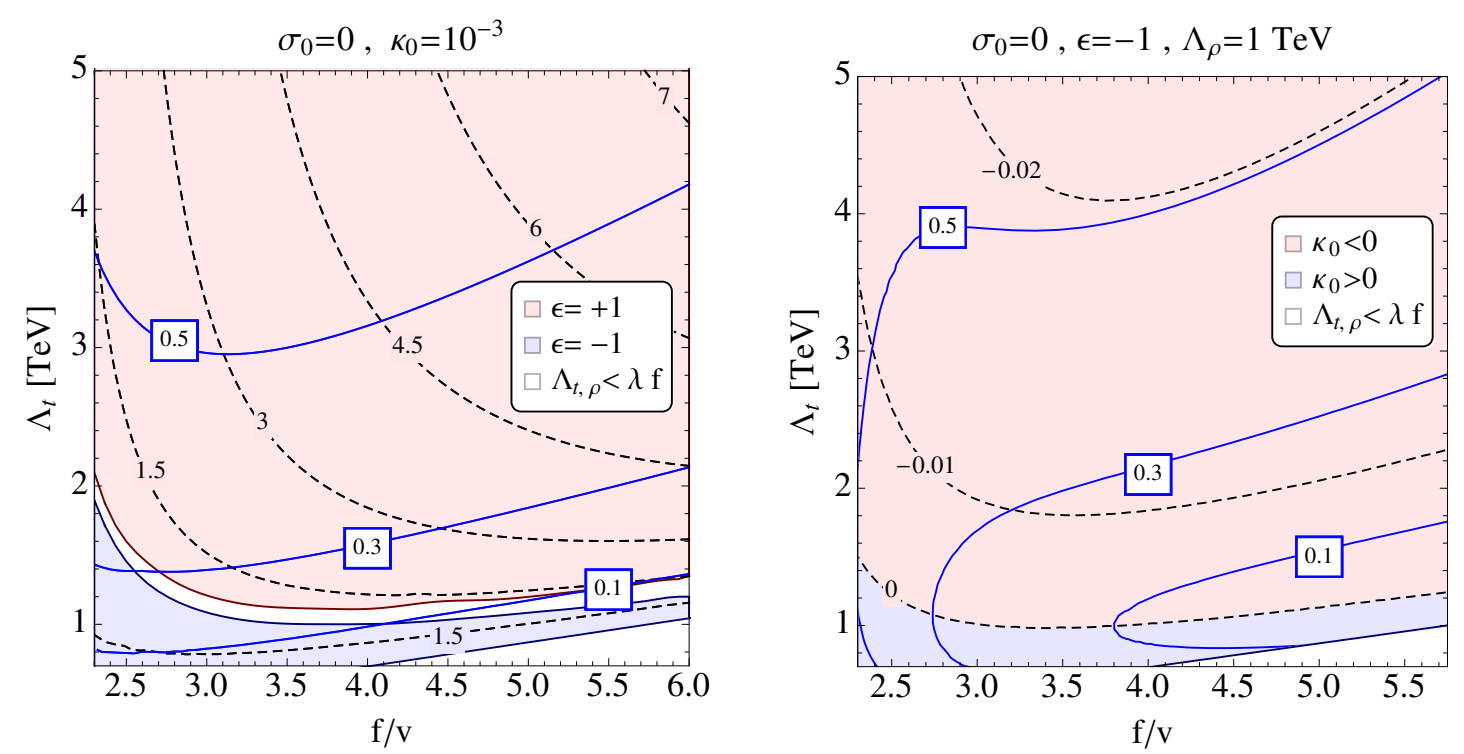

Figure 4. The solid blue lines in both plots indicate the gain in fine-tuning of the hard-breaking model with respect to the soft one. In the white region $\Lambda_{t}<\lambda f$ or $\Lambda_{\rho}<\lambda f$ with $\lambda=1$. Left: we take $\kappa_{0}=10^{-3}$ and the black dashed contours show the $\epsilon \times \Lambda_{\rho}$ (in $\mathrm{TeV}$ units) adjusted to obtain $m_{h}=125 \mathrm{GeV}$. The light red/blue region is the allowed parameters space with $\epsilon= \pm 1$. Right: we fix $\epsilon=-1$ and $\Lambda_{\rho}=1 \mathrm{TeV}$ and fix $\kappa_{0}$ (black dashed contours) to get $m_{h}=125 \mathrm{GeV}$. The light red/blue region is the allowed parameters space with $\kappa_{0} \lessgtr 0$.

This leads to a new contribution to the fine-tuning from the adjustment of $\kappa_{0}$ against $\Delta \kappa$ that we sum up in quadrature with eq. (2.12), and the final gain in fine-tuning with respect to the soft breaking scenario is indicated by the blue isolines of figure 4 (right).

We now comment on the caveats of our effective analysis. First, the factorization of the fine-tuning measure very often fails in UV complete models. This failure is related to RGE effects that introduce a dependence of $f$ on the $\mathbb{Z}_{2}$-breaking parameters. These effects could be important in UV complete hard breaking models as we will see in section 3 . Second, there is the log-dependence of both $\kappa$ and $\sigma$ on $\Lambda_{\rho}$, which we have neglected in order to have a simple analytical understanding. Third there might be extra threshold corrections coming from the UV theory which sensibly affect the Higgs mass estimate. All these effects can slightly weaken our naive estimates of the reduction in fine-tuning.

In the next section we will show how all these caveats are taken under control in explicit SUSY UV completions, which indeed follow the parametrical intuition developed here in most of the parameter space. In particular we will show examples of hard breaking models that sensibly ameliorate the fine-tuning of the Twin Higgs.

\section{SUSY UV completions}

In this section we discuss simple SUSY UV completions of the Twin Higgs. In the minimal setup the Twin SUSY UV completion consists of two copies of the MSSM $\left(\operatorname{MSSM}_{A} \times\right.$ $\operatorname{MSSM}_{B}$ ) that are symmetric under the action of the $Z_{2}$ exchange symmetry $A \leftrightarrow B$. The 
Higgs sector of the model consists of two replicas of the two Higgs doublet model charged under the $A$ and the $B$ EW group respectively.

Even if the most general SUSY potential is quite involved, we can develop an analytical understanding by going in the decoupling limit of the SUSY Higgses and matching it to the non-SUSY Twin Higgs potential in eq. (2.1) (with 2 doublets $H_{A, B}$ ) by replacing

$$
\begin{array}{ll}
h_{u}^{A}=H_{A} s_{A}, & h_{u}^{B}=H_{B} s_{B}, \\
h_{d}^{A}=H_{A}^{\dagger} c_{A}, & h_{d}^{B}=H_{B}^{\dagger} c_{B},
\end{array}
$$

where $s_{A, B}=\sin \beta_{A, B}, c_{A, B}=\cos \beta_{A, B}$. Of course this matching gives a good description of the SUSY Twin Higgs EWSB as long as the SUSY Higgses are heavier than the Twin Higgs. We give a treatment of the full four Higgs doublet model beyond this approximation in appendix B.

We first review Twin SUSY constructions with only soft $\mathbb{Z}_{2}$-breaking, which exemplifies several generic features of the SUSY UV completions of the Twin Higgs. We pay particular attention to the simplest soft Twin SUSY model proposed in ref. [20] and validate our analytical understanding against a full numerical treatment. We show how the parameter space of this model can be completely solved once the Higgs mass and EWSB constraints are imposed and comment on possible directions to ameliorate the fine tuning.

Then we move to Twin SUSY models with hard $\mathbb{Z}_{2}$-breaking, which UV-complete some previously described setups and feature similar improvement in the FT. We first discuss the prominent role of the Higgs mass constraint in these models showing that the simplest implementation of hard $\mathbb{Z}_{2}$-breaking in SUSY has a strong upper bound on the stop mass scale $M_{s}{ }^{10}$ once the constraint $m_{h}=125 \mathrm{GeV}$ is imposed. Finally, we present an explicit model where the measured Higgs mass is obtained via a negative contribution to $\kappa$, very much in the spirit of the effective theory presented in the right panel of figure 4 . In this model we get $\sim 10 \% \mathrm{FT}$ with $M_{s} \sim 2 \mathrm{TeV}$ and $\lambda_{S}=1$, ameliorating the fine-tuning of soft Twin-SUSY by a factor of about $\sim 5$.

\subsection{Soft Twin SUSY}

In this subsection we analyze SUSY UV completions of the Twin Higgs with softly broken mirror symmetry. Even though this kind of UV completions have already been discussed to some extent in the literature, reviewing them carefully will clarify the basic building blocks of any SUSY UV completion of the Twin Higgs. Analogously to section 2, we organize our discussion according to the global symmetries that each term of the scalar potential preserves.

Generating the SU(4)-invariant part of the Twin Higgs potential in eq. (2.1) already introduces some degree of model dependence in SUSY UV completions. Indeed, if we consider the matter content of just two copies of the MSSM, the only SU(4)-preserving operators at the renormalizable level are the mass terms. Therefore the SU(4)-invariant quartics require some additional dynamics. The simplest possibility is to use a non-decoupling F-term

\footnotetext{
${ }^{10}$ Hereafter we define the stop mass scale as a geometric mean of the stop masses $M_{s} \equiv \sqrt{M_{\tilde{t}_{1}} M_{\tilde{t}_{2}}}$. Note also that $M_{\tilde{t}_{i}}$ stands for the physical mass of the stop, rather than for its soft mass.
} 
from a heavy singlet $S$ that has NMSSM-like couplings with the $A$ and $B$ Higgses, as in refs. $[18,20]$. The superpotential and soft masses of this setup are

$$
\begin{aligned}
W_{\mathrm{SU}(4)} & =\left(\mu+\lambda_{S} S\right) \mathcal{H}_{u} \mathcal{H}_{d}+\frac{\mathcal{M}_{S}}{2} S^{2}, \\
V_{\mathrm{SU}(4)} & =m_{H_{u}}^{2}\left|\mathcal{H}_{u}\right|^{2}+m_{H_{d}}^{2}\left|\mathcal{H}_{d}\right|^{2}-b\left(\mathcal{H}_{u} \mathcal{H}_{d}+\text { h.c. }\right)+m_{S}^{2}|S|^{2} .
\end{aligned}
$$

To make our equations more compact, we have switched here to manifestly $\mathrm{SU}(4)$ invariant notations. We will further use $\mathcal{H}_{u, d}=\left(h_{u, d}^{A}, h_{u, d}^{B}\right)$, wherever the SU(4) conventions are appropriate, and with a slight abuse of notation, we will use $\mathcal{H}_{u, d}$ both for the Higgs superfields and their lowest components.

We also assume that the singlet soft mass is much larger than the SUSY one and integrate out $S$ in this limit. The potential we get is:

$$
V_{\mathrm{SU}(4)}^{\mathrm{eff}} \approx m_{u}^{2}\left|\mathcal{H}_{u}\right|^{2}+m_{d}^{2}\left|\mathcal{H}_{d}\right|^{2}-b\left(\mathcal{H}_{u} \mathcal{H}_{d}+\text { h.c }\right)+\lambda_{S}^{2}\left|\mathcal{H}_{u} \mathcal{H}_{d}\right|^{2}
$$

where we have defined $m_{u, d}^{2}=\mu^{2}+m_{H_{u, d}}^{2}$. We also kept just the renormalizable operators and neglected the extra quartics of order $\mathcal{O}\left(\mathcal{M}_{S}^{2} / m_{S}^{2}\right), \mathcal{O}\left(\mu \mathcal{M}_{S} / m_{S}^{2}\right)$ and $\mathcal{O}\left(\mu^{2} / m_{S}^{2}\right)$. By construction these sub-leading quartic terms are also SU(4)-invariant.

Throughout this paper we will often trade the $b$-term for the mass of the heavy CPodd Higgs $m_{A_{T}}=2 b / \sin (2 \beta)$. Note, that unlike in the MSSM, where $2 b / \sin (2 \beta)$ is the mass-squared of the CP-odd Higgs, here, in the SUSY Twin Higgs, it controls the masssquared of the mirror CP-odd Higgs. The mass of the "visible" CP-odd Higgs turns out to be always lighter: $m_{A}^{2} \approx m_{A_{T}}^{2}-\lambda_{S}^{2} f^{2}$ (see appendix B).

We further match the SU(4)-invariant parameters of the SUSY potential in eq. (3.3) to the parameters of the Twin Higgs potential in eq. (2.1):

$$
\lambda \approx \frac{\lambda_{S}^{2}}{4} s_{2 \beta}^{2}, \quad m^{2} \approx m_{u}^{2} s_{\beta}^{2}+m_{d}^{2} c_{\beta}^{2}-b s_{2 \beta} .
$$

In these expressions we have disregarded the terms that depend on the difference between the $\beta$ angles in the different sectors. We will later analyze in detail the role of the $\beta$ misalignment. So far we are taking the SUSY decoupling limit, which is defined by eq. (3.1), while we are keeping the twin Higgs in the spectrum treating the Twin Higgs model as a full linear sigma model. The effective PGB theory discussed in section 2 can be obtained integrating out the twin Higgs and expanding at the first non-trivial order in $\lambda \gg \kappa, \sigma$ (see appendix B for details on this point).

Eq. (3.4) immediately explains the origin of the problem already mentioned in the previous section: SUSY UV completions have a hard time to maximize $\lambda$ and therefore also the fine-tuning gain, which is $\lambda_{\mathrm{SM}} / \lambda$. In SUSY UV completions $\lambda_{S}$, rather than $\lambda$, should be perturbative. Moreover, as we will shortly see, in order to get the right Higgs mass we will have to stick to moderate values of $\tan \beta$, thus further suppressing the effective $\lambda$. As a result, we will generally get $\lambda<1$, such that the gain in fine tuning will never be large. In passing, we notice that the fine tuning in the soft models can be slightly ameliorated by changing the functional dependence of $\lambda$ on $\tan \beta$ and other fundamental parameters, as we comment later on. 
We now proceed to discus the leading $\mathbb{Z}_{2}$-even but $\mathrm{SU}(4)$-breaking operators in the Twin SUSY potential. At leading order the SU(4)-breaking originates at tree-level from the electroweak D-terms and at one-loop from the top Yukawa sector:

$$
\begin{aligned}
V_{\Psi(4)}^{D} & =\frac{g_{\mathrm{ew}}^{2}}{8}\left[\left(\left|h_{u}^{A}\right|^{2}-\left|h_{d}^{A}\right|^{2}\right)^{2}+\left(\left|h_{u}^{B}\right|^{2}-\left|h_{d}^{B}\right|^{2}\right)^{2}\right], \\
V_{\Psi(4)}^{\mathrm{top}} & \approx \frac{3 y_{t}^{4}}{16 \pi^{2}}\left[\left(\left|h_{u}^{A}\right|^{4}+\left|h_{u}^{B}\right|^{4}\right) \log \frac{M_{s}^{2}}{m_{t_{B}}^{2}}+\left|h_{u}^{A}\right|^{4} \log \frac{f^{2}}{v^{2}}\right],
\end{aligned}
$$

where we defined $g_{\mathrm{ew}}^{2}=g^{2}+g^{\prime 2}$, and $y_{t}$ is the SUSY superpotential coupling, related to the top mass as $m_{t}=y_{t} v \sin \beta$. In order to get eq. (3.6) we compute the CW potential and and set the dynamical Higgses to their VEVs in the non-polynomial terms. The RH and LH stops mass are assumed to be equal, as well as the soft masses in the $A$ and $B$ sectors. ${ }^{11}$

Matching again the effective SU(4)-breaking potential to eq. (2.1) we identify

$$
\kappa \approx \frac{g_{\mathrm{ew}}^{2}}{8} c_{2 \beta}^{2}+\frac{3 m_{t}^{4}}{16 \pi^{2} v^{4}} \log \left(\frac{M_{s}^{2}}{m_{t}^{2}} \frac{v^{2}}{f^{2}}\right) .
$$

This expression indicates that the $\mathrm{SU}(4)$-breaking $\mathbb{Z}_{2}$-even quartic $\kappa$ gets an unavoidable positive contribution from the EW D-terms at tree level in any SUSY UV completion. The ballpark of this contribution is $\mathcal{O}\left(10^{-2} \ldots 10^{-3}\right)$ depending on $\tan \beta$. The contribution from the top-stop sector reproduces the result obtained in eq. (2.5). Given that top loops alone already set the Higgs mass in the right ballpark (see the blue contours in figure 2), we expect that for a fixed $M_{s}$ a Twin SUSY model would have an upper bound on $\tan \beta$. We will later see that because of this bound only models with small or moderate $\tan \beta$ are viable.

In those Twin SUSY theories, where the mirror symmetry is broken only softly, the $\mathbb{Z}_{2}$-breaking terms can be introduced as soft masses in the potential:

$$
V_{\mathbb{R}_{2}}^{\text {soft }} \approx \Delta m_{u}^{2}\left|h_{u}^{A}\right|^{2}+\Delta m_{d}^{2}\left|h_{d}^{A}\right|^{2}+\Delta b\left(h_{u}^{A} h_{d}^{A}+\text { h.c }\right) .
$$

These can easily be matched to the respective term in the IR effective potential in eq. (2.1)

$$
\sigma \approx \sigma_{u} s_{\beta}^{2}+\sigma_{d} c_{\beta}^{2}
$$

where we defined $\sigma_{u, d}=\Delta m_{u, d}^{2} / f^{2}$ and assume $\Delta b=0$.

Using the matching conditions described so far, we can rephrase in SUSY language the two EWSB conditions, which were already captured by the low energy Twin Higgs potential in eq. (2.1). The first EWSB condition is the one we discussed in the PGB limit in eq. (2.3) which fixes the EW scale $v$ in terms of $f$. This is achieved in soft $\mathbb{Z}_{2}$-breaking models by balancing $\sigma$ against $\kappa$. These two parameters are determined in terms of four parameters of the SUSY theory: $\beta$ and $M_{s}$ determine $\kappa$ through eq. (3.7), while $\sigma_{u}, \sigma_{d}$ and $\beta$ give $\sigma$ through eq. (3.9).

\footnotetext{
${ }^{11}$ We also assume the trilinear $A$-terms to be negligible and expand these expressions at the leading order in $m_{t_{A, B}}^{2} / M_{s}^{2}$.
} 
Solving the EWSB condition in the IR effective theory we can trade $\sigma$ for $f / v$. The second combination of $\sigma_{u}$ and $\sigma_{d}$ is unimportant in the $\delta t_{\beta}=0$ limit. Imposing the Higgs mass constraint in the PGB limit then fixes $\kappa$ and allows us to predict the value of $\beta$ in the $\left(f / v, M_{s}\right)$ plane by solving eq. (3.7) for $\beta$. This procedure holds only as far as $\kappa / \lambda \ll 1$ and we will later discuss how the Higgs mass formula gets modified beyond the PGB approximation.

The second condition is essentially the $\mathrm{SU}(4)$ breaking condition, namely $f^{2} \approx m^{2} / 2 \lambda$, rephrased in terms of the parameters of the underlining SUSY theory (note that here $m$ stands for the mass parameter in eq. (2.1)) using the matching conditions in eq. (3.4). The dependence of the scale $f$ on the stop threshold $M_{s}$ can then be estimated via the RGE of $m_{H_{u}}$, which largely dominates the fine-tuning measure. In this approximation we get that $\delta f^{2} \approx \frac{2 s_{\beta}^{2}}{\lambda_{S}^{2} s_{2 \beta}^{2}} \times \delta m_{H_{u}}^{2}$. The fine tuning measure derived in eq. (2.11) in terms of the SUSY parameters is

$$
\Delta^{\text {soft }} \approx \frac{3 y_{t}^{2} s_{\beta}^{2}}{2 \pi^{2} \lambda_{S}^{2} s_{2 \beta}^{2}} \frac{M_{s}^{2} \log \frac{\Lambda_{S U S Y}}{M_{s}}}{2 v^{2}} .
$$

In this estimate we also neglect the contribution of the singlet soft mass $m_{S}$ in the RGE for $m_{H_{u}}$. This contribution turns out to be sub-leading with respect to the stops one as long as $m_{S} \sim 1 \ldots 2 \mathrm{TeV}$.

Looking back at the low energy fine-tuning (2.11) we see that in the SUSY theory $\Lambda_{t}^{2}$ is identified with $2 M_{s}^{2} \times \log \frac{\Lambda_{S U S Y}^{2}}{M_{s}^{2}}$. The UV cut-off $\Lambda_{S U S Y}$ can be interpreted as a scale where the soft masses are formed, corresponding for example to the messenger scale in low energy SUSY-breaking scenarios. For the purpose of numerical calculations we will further use $\Lambda_{S U S Y}=100 M_{s}$.

All in all the gain in fine tuning of soft Twin SUSY with respect to standard SUSY models like the NMSSM is

$$
\frac{\Delta^{\mathrm{soft}}}{\Delta^{\mathrm{NMSSM}}} \approx \frac{m_{h}^{2}}{2 v^{2}} \times \frac{1}{\lambda_{S}^{2} s_{2 \beta}^{2}}=\frac{2 \lambda_{\mathrm{SM}}}{\lambda_{S}^{2} s_{2 \beta}^{2}},
$$

in agreement with the analysis performed in ref. [20] as well as with our expectations from the IR effective theory analysis in section 2. It is important to remember that the Higgs mass constraint in this model fixes the value of $\beta$ for a given $M_{s}$ and $f / v$ determining the final gain in fine tuning.

We summarize the behavior of the soft Twin SUSY model in figure 5. We work directly in the SUSY theory, solve numerically the EWSB conditions and the Higgs mass constraint, and present the allowed parameter space in the $\left(f / v, M_{s}\right)$ plane. In this plane we show the behavior of the fine tuning measure, the minimal value of $\tan \beta$ that satisfies the Higgs mass constraint ${ }^{12}$ and the mass of the next-to-lightest CP-even Higgs. We show our results for two different choices of the mirror CP-odd Higgs masses $m_{A_{T}}=1.5,3 \mathrm{TeV}$. In the rest of the section we give an analytical understanding of the results of figure 5 .

\footnotetext{
${ }^{12}$ Since we are allowing for a range of possible SM Higgs masses we select the minimal tan $\beta$ within the range of the allowed ones.
} 

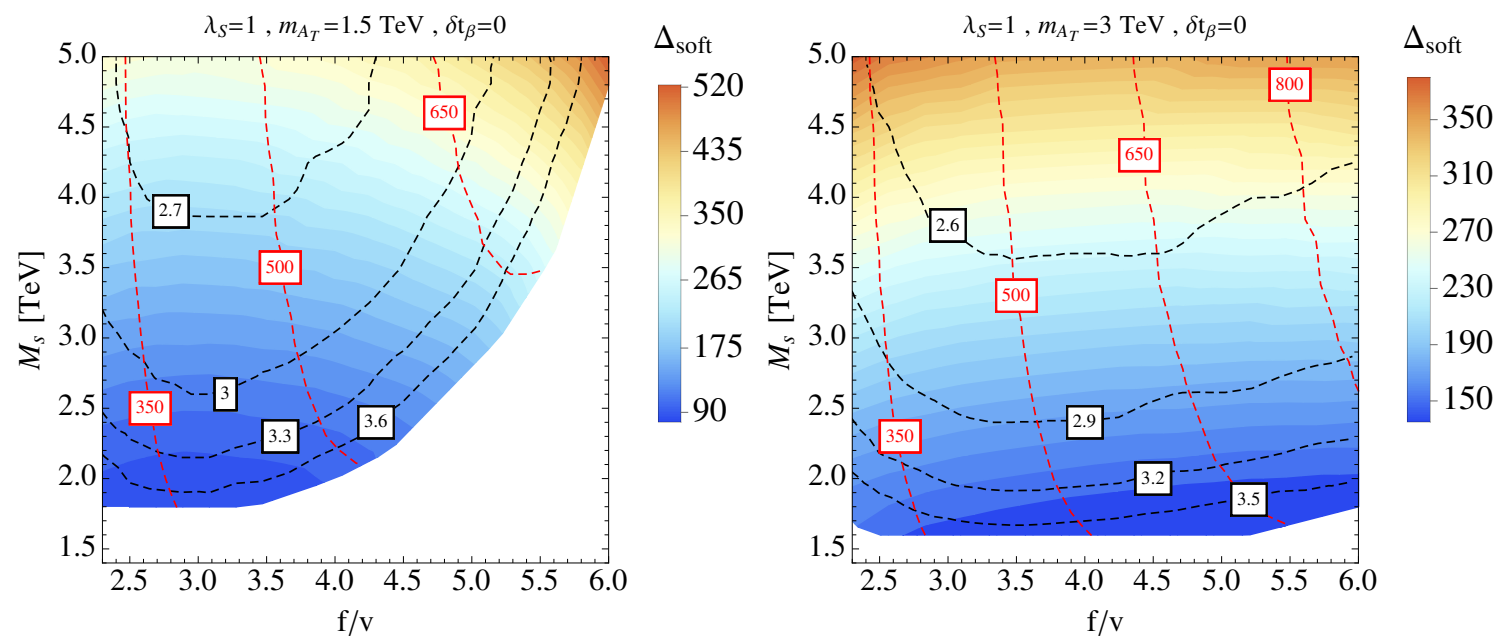

Figure 5. Soft Twin SUSY parameter space with $m_{A_{T}}=1.5 \mathrm{TeV}$ (left panel) and $m_{A_{T}}=3 \mathrm{TeV}$ (right panel). In the white region $m_{h}=125 \pm 2 \mathrm{GeV}$ cannot be achieved as explained in the text. We also assume here $\delta t_{\beta}=0, \lambda_{S}=1$ and $m_{S}=1 \mathrm{TeV}, \mu=500 \mathrm{GeV}, M_{3}=M_{s}$. Black $/$ red dashed lines are contours of $\tan \beta$ /next-to-lightest CP-even Higgs mass in $\mathrm{GeV}$. The gradient color function indicates region of increasing $\Delta_{\text {soft }}$, calculated in eq. (3.12).

The fine tuning we show in figure 5 is computed à la Giudice-Barbieri by evaluating numerically the logarithmic derivatives of the EW scale $v$ with respect to all the UV parameters of the full soft Twin SUSY model:

$$
\Delta^{\mathrm{soft}}=\sqrt{\sum_{i=\mathcal{P}_{\text {soft }}}\left(\frac{\partial \log v^{2}}{\partial \log p_{i}}\right)^{2}} .
$$

In this expression $i$ runs over the parameters of the Higgs sector $\mathcal{P}_{H}=\left\{m_{H_{u}}^{2}, m_{H_{d}}^{2}, \mu^{2}, b\right\}$, of the colored sector $\mathcal{P}_{Q}=\left\{m_{Q}^{2}, m_{U}^{2}, M_{3}\right\}$, of the singlet sector $\mathcal{P}_{S}=\left\{m_{S}^{2}, \lambda_{S}\right\}$, and of the $\mathbb{Z}_{2}$-breaking sector $\mathcal{P}_{\mathbb{Z}_{2}}=\left\{\Delta m_{u}^{2}, \Delta m_{d}^{2}\right\}$. All these parameters are considered at the "messenger scale" $\Lambda_{S U S Y}$ for the purpose of the fine-tuning computation and we use full one-loop RGE (see appendix A) to obtain the final fine tuning.

One more comment is appropriate here regarding the total fine tuning measure in eq. (3.12) that we are using. This is slightly different from the measure which has been used by Barbieri and Giudice in [45], which assumed the total fine-tuning to be the maximum of the one-parameter fine tunings, rather than their sum in quadrature, as we do. We choose this particular measure to give a numerical expression to an intuition, that if a model has a similar fine-tuning with respect to two independent parameters, it is more fine-tuned, than a model that is one-tuned only with respect to a single parameter. We checked it explicitly, and in vast majority of the parameter space our measure is not very different numerically from the nominal Barbieri-Giudice measure. Note however, that in case of multiple free parameters and small fine-tuning our measure overestimates the fine-tuning with respect to the Barbieri-Giudice measure.

As expected, our tuning measure can be well approximated by formula (3.10), meaning that the gross features of the Twin SUSY model are captured by the PGB intuition we 
have developed so far. In particular the factorized formula for the tuning measure works pretty accurately and we can rewrite eq. (3.12) as

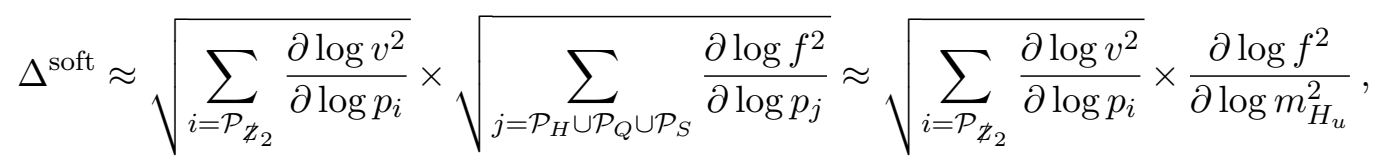

where the last expression exactly reproduces the approximated formula in eq. (3.10).

The gain in fine tuning with respect to the NMSSM (3.11) follows essentially the contours of $\tan \beta$ in figure 5 . Since $\tan \beta$ decreases at larger $M_{s}$, the effective $\lambda$ grows (see eq. (3.4)), and consequently this particular soft Twin SUSY model has a bigger gain with respect to the NMSSM at higher values of $M_{s}$. For $\lambda_{S}=1$ the gain is merely a factor of $0.4 \ldots 0.5$, depending on the region of the parameter space. This can be slightly improved up to $0.2 \ldots 0.3$ by taking $\lambda_{S}=1.4$, i.e. very close to its perturbativity bound.

The fine tuning measure is a relatively flat function of $f / v$ as expected from the factorized formula (3.10). This is especially true in the right panel where we take $m_{A_{T}}=$ $3 \mathrm{TeV}$ and the SUSY states are completely decoupled in the full range of $f / v$. The residual dependence on $f / v$ in the left panel is introduced indirectly by the Higgs mass constraint, which in turn determines the value of $\tan \beta$ at fixed $f / v$ and $M_{s}$.

We now analyze the role of the Higgs mass in the soft Twin SUSY parameter space. In particular, we would like to understand the absolute lower bound on $M_{s}$ which is present in both plots and the upper bound on $f / v$ at fixed $M_{s}$ in the left panel. These two bounds are responsible for the white region in figure 5 , where there is no value of $\tan \beta$ for which the Higgs mass constraint can be satisfied. Assuming that there is an upper bound on $\tan \beta$, the lower bound on $M_{s}$ can be easily understood from the PGB mass formula:

$$
m_{h}^{2} \approx g_{\mathrm{ew}}^{2} v^{2} c_{2 \beta}^{2}+\frac{3 m_{t}^{4}}{2 \pi^{2} v^{2}} \log \left(\frac{M_{s}^{2}}{m_{t}^{2}} \frac{v^{2}}{f^{2}}\right),
$$

which shows that for most of the moderate $\tan \beta$ we undershoot $m_{h}=125 \mathrm{GeV}$ unless we enhance the radiative corrections, yielding a lower bound on the $M_{s}$.

To understand the upper bound on $\tan \beta$ we have to go beyond the PGB approximation, which breaks down for large $\tan \beta$, because the PGB approximation requires

$$
\frac{\kappa}{2 \lambda} \ll 1 \quad \Longrightarrow \quad\left(\frac{g_{\mathrm{ew}}}{2 \lambda_{S}}\right)^{2} \cot ^{2} 2 \beta \ll 1
$$

Beyond the PGB approximation we should take into account all the $\kappa / \lambda$ corrections, which is not easy to do analytically (see however appendix B). Nonetheless, we can arrive to the right conclusions analyzing the first non-vanishing correction to the PGB approximation:

$$
m_{h}^{2} \approx 8 \kappa v^{2}\left(1-\frac{v^{2}}{f^{2}}\right)\left(1-\frac{\kappa}{2 \lambda}+\ldots\right) \approx g_{\mathrm{ew}}^{2} v^{2} \cos ^{2} 2 \beta\left(1-\frac{g_{\mathrm{ew}}^{2}}{4 \lambda_{s}^{2}} \cot ^{2} 2 \beta\right) .
$$

As $\tan \beta$ grows, the term in the brackets becomes important, and drives the Higgs mass down, signalling that in the full Higgs mass we have a "sweet spot" for the $\beta$ angle at which we can maximize the Higgs mass, and therefore the minimal allowed value for $M_{s}$. 



Figure 6. The Higgs mass for $M_{s}=2 \mathrm{TeV}$ and $\lambda_{S}=1$ as a function of $\tan \beta$ (left) and $f / v$ (right). Blue and red line correspond to $m_{A_{T}}=1.5,3 \mathrm{TeV}$ respectively. In the yellow band we get $m_{h}=125 \pm 2 \mathrm{GeV}$.

We show the value of the Higgs mass as a function of $\tan \beta$ and $f / v$ using the full numerical calculation at all orders in $\kappa / \lambda$ on the left panel figure 6 . This confirms that the full function of the Higgs mass has a sweet spot for $\tan \beta$, in qualitative agreement with the simplified formula in eq. (3.16).

The upper bound on $f / v$ at fixed $M_{s}$ instead strongly depends on the value of $m_{A_{T}}$ which controls the masses of the SUSY Higgses (see table 1 and appendix B for details). In particular we see in figure 5 that the upper bound on $f / v$ disappears for $m_{A_{T}}=3 \mathrm{TeV}$. This feature is also illustrated on the right panel of figure 6 and can be understood as a consequence of level splitting between the SM Higgs and the CP-even SUSY Higgs whose mass squared is proportional to $\propto\left(m_{A_{T}}^{2}-\lambda_{S}^{2} f^{2}\right)$. For fixed $m_{A_{T}}$ increasing $f / v$ reduces the SUSY Higgs, thus enhancing the level splitting and decreasing the SM Higgs mass. This effect can always be compensated by taking a larger $m_{A_{T}}$ (see figure 6), however this comes at the price of an $\mathcal{O}(1)$ increasing of the overall fine-tuning for $M_{s} \lesssim 3 \mathrm{TeV}$.

Interestingly, an indirect bound on $f / v$ at fixed $M_{s}$ implies an upper bound on the twin Higgs mass. Since the twin Higgs is the radial mode of the SU(4)-charged Higgs, its mass is roughly $\sim \lambda_{S} f$ up to the $\kappa / \lambda$ corrections. For example, for $M_{s}=2 \mathrm{TeV}$ and $m_{A_{T}}=1.5 \mathrm{TeV}$ the twin Higgs cannot exceed $500 \mathrm{GeV}$ and can be probed at the LHC as we will discuss in more detail in section 4 .

Up to now we completely ignored the role of $\delta t_{\beta}$ which we set to zero in figure 5 for simplicity. However, in the full SUSY theory there are two more relations which determine $\tan \beta$ and the misalignment between the $\beta$ angles in terms of the fundamental parameters (at the leading order in $\delta t_{\beta}$ ):

$$
t_{\beta}^{2} \approx \frac{m_{d}^{2}}{m_{u}^{2}}, \quad \delta t_{\beta} \approx \frac{2 f^{2}}{m_{A}^{2} s_{2 \beta}}\left[2\left(1-2 \frac{v^{2}}{f^{2}}\right) \frac{g_{\mathrm{ew}}^{2}}{8}\left|c_{2 \beta}\right|+\sigma_{d}\right]
$$

where $m_{A}^{2}=m_{A_{T}}^{2}-\lambda_{S}^{2} f^{2}$ is the mass of the MSSM-like CP-odd Higgs. 

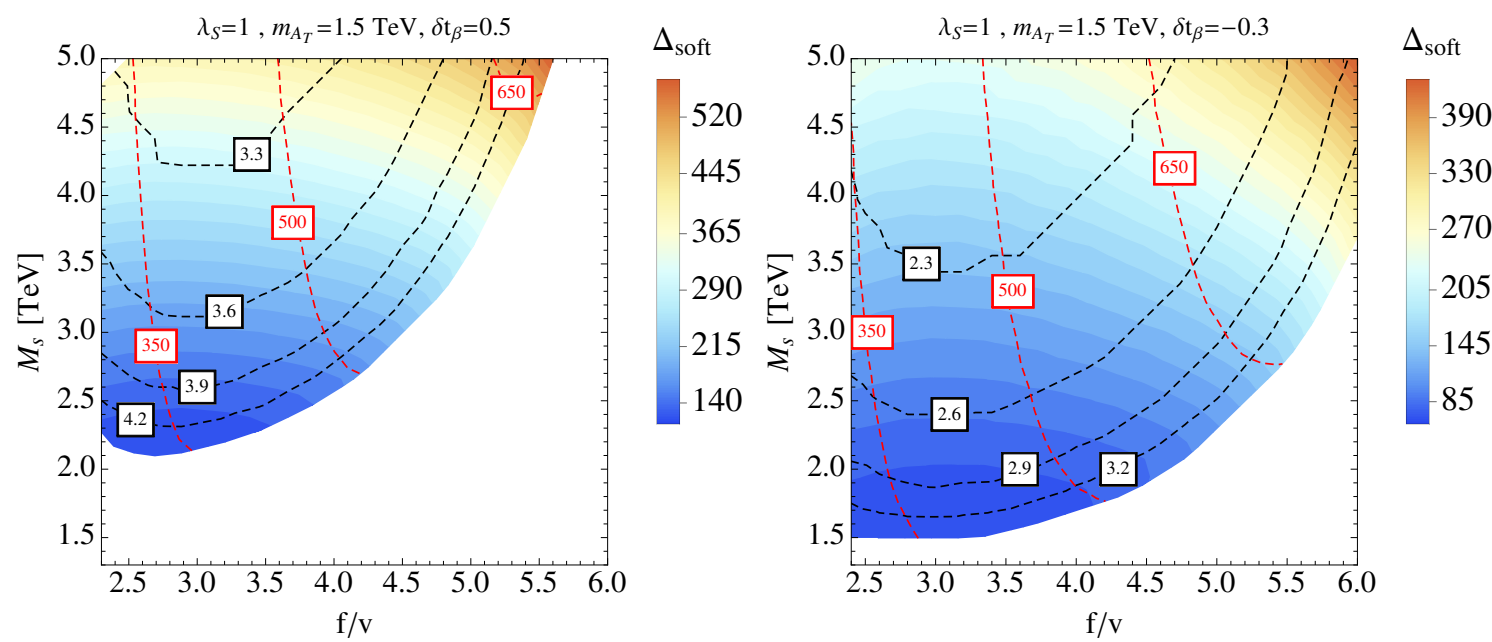

Figure 7. Soft Twin SUSY parameter space with $m_{A_{T}}=1.5 \mathrm{TeV}$ and $\delta t_{\beta}=0.5$ (left panel) and $\delta t_{\beta}=-0.3$ (right panel). In the white region $m_{h}=125 \pm 2$ cannot be achieved as explained in the text. We also fix $\lambda_{S}=1$ and $m_{S}=1 \mathrm{TeV}, \mu=500 \mathrm{GeV}, M_{3}=M_{s}$. Black/red dashed lines are contours of $\tan \beta /$ next-to-lightest $\mathrm{CP}$-even Higgs mass in $\mathrm{GeV}$. The gradient color function indicates region of increasing $\Delta_{\text {soft }}$, which is defined in eq. (3.12).

From these expressions one can estimate what would be the natural value for the $\beta$ angle misalignment between the different sectors. If we require no unnatural cancellations and the "sweet spot" values for $\tan \beta$ (namely of order $2 \ldots 3$ ), we expect that $\left|\delta t_{\beta}\right| \lesssim 1$, but usually not smaller than 0.1 . Because these natural values are not necessarily small, we show in figure 7 how the non-trivial $\beta$-angle misalignment changes the allowed parameter space and the fine-tuning. Negative values of $\delta t_{\beta}$ are allowed, because $\sigma_{d}$ can be either positive or negative, allowing to extend the parameter space at larger $f / v$. A positive $\delta t_{\beta}$ makes instead the parameter space shrink. The qualitative features of the plots are however left unchanged with respect to the $\delta t_{\beta}=0$ case.

We conclude that this particular soft Twin SUSY model has a number of features which crucially depend on the fact that $\lambda$ is generated via the non-decoupling F-term of a NMSSM-like singlet and hence suppressed even for moderate $\tan \beta$. In particular the $\tan \beta$-dependence of $\lambda$ renders the behavior of the tuning measure sub-optimal and certainly motivates further exploration in the theory space of soft $\mathbb{Z}_{2}$-breaking models. For example, one can consider models along the lines of [46] where an extra $\mathrm{U}(1)_{x}$ is gauged and both $A$ and $B$ Higgses carry the same $\mathrm{U}(1)_{x}$ charge. If the $\mathrm{U}(1)_{x}$ is spontaneously broken by fields with large soft masses, one would obtain a non-decoupling $\mathrm{SU}(4)$-symmetric quartic from the D-terms of the $\mathrm{U}(1)_{x}$, getting a very different dependence of the effective $\lambda$ on the $\beta$-angle. We leave an exploration of soft Twin SUSY models for future work.

Let us finally mention some caveats of our analysis. First, the allowed parameter space presented in figure 5 depends crucially on our requirement $m_{h}=125 \pm 2 \mathrm{GeV}$. This range is probably too conservative since our one-loop computation is subject to large theoretical uncertainties dominated by two-loop QCD effects. It is known that these effects can sometimes shift the one-loop result by more than $2 \mathrm{GeV}$ in SUSY theories where they 
have been already computed like in the MSSM. In particular a large positive shift from two loop effect might quantitatively change our conclusions on the allowed parameter space of the model. Nevertheless our analysis illustrates some important features of tree level effects to the Higgs mass that will certainly be present also after the inclusion of two loops corrections.

Second, an interesting option to enlarge the parameter space of this particular soft Twin SUSY model and possibly ameliorate its fine tuning would be to introduce a sizeable negative $\delta t_{\beta}$. This value does not come out naturally, but might be justified in a UV complete model where the origin of both $\mathbb{Z}_{2}$-even and $\mathbb{Z}_{2}$-odd soft masses is specified. We leave these questions open for future work.

\subsection{Hard Twin SUSY}

In this section we discuss models of Twin SUSY where a $\mathbb{Z}_{2}$-breaking quartic is generated at the tree-level. The easiest implementation of this class of models borrows the SU(4)invariant sector from the soft model described above and just extends the singlet sector adding a new singlet $S_{A}$ which couples to the $A$-sector Higgses only

$$
W_{\mathbb{R}_{2}}^{\text {hard }}=\lambda_{A} S_{A} H_{u}^{A} H_{d}^{A}+\frac{\mathcal{M}_{S_{A}}}{2} S_{A}^{2}, \quad \quad V_{\mathbb{Z}_{2}}^{\text {hard }}=m_{S_{A}}^{2}\left|S_{A}\right|^{2} .
$$

Integrating out the singlet in the limit $m_{S_{A}} \gg \mathcal{M}_{S_{A}}, \mu$ we generate a $\mathbb{Z}_{2}$-breaking quartic at treel level while the $\mathbb{Z}_{2}$-breaking soft masses in eq. (3.8) are generated at 1-loop:

$$
V_{\mathbb{Z}_{2}}^{\text {hard }} \approx \lambda_{A}^{2}\left|h_{u}^{A} h_{d}^{A}\right|^{2}-\frac{3 \lambda_{A}^{2} m_{S_{A}}^{2}}{32 \pi^{2}}\left(\left|h_{u}^{A}\right|^{2}+\left|h_{d}^{A}\right|^{2}\right),
$$

with the same approximations that we have used before and neglecting the log-pieces in the CW potential. Matching eq. (3.19) to the $\mathbb{Z}_{2}$-odd parameters of the Twin Higgs potential in eq. (2.1) we get

$$
\rho \approx \frac{\lambda_{A}^{2}}{4} s_{2 \beta}^{2}, \quad \sigma \approx-\frac{3 \lambda_{A}^{2}}{32 \pi^{2}} \frac{m_{S_{A}}^{2}}{f^{2}} \equiv-\lambda_{A}^{2} \sigma_{S_{A}} .
$$

After comparing these matching conditions with the results of section 2 we see that the required values of $\mathbb{Z}_{2}$-breaking quartic $\rho \gtrsim 0.1$ to get $f / v \gtrsim 2.3$ are easy to achieve with a moderate coupling $\lambda_{A} \gtrsim 0.6$ as long as $\tan \beta \lesssim 2$.

The biggest problem of this model is that the one-loop threshold corrections to $\sigma$ are negative and their size is controlled by the singlet mass. A negative $\sigma$ is subject to a strong upper bound coming from the stability of the EWSB vacuum (cf. figure 1 and the discussion in section 2). In a SUSY UV completion we get a similar bound by solving the EWSB condition for $f / v$ in terms of $\lambda_{A}$

$$
\lambda_{A}^{2} \approx-\frac{1-\frac{2 v^{2}}{f^{2}}}{\frac{\sigma_{S_{A}}}{2}-\frac{v^{2}}{4 f^{2}} s_{2 \beta}^{2}} \kappa .
$$

Since $\lambda_{A}^{2}>0$ we need $\frac{\sigma_{S_{A}}}{2}<\frac{v^{2}}{4 f^{2}} s_{2 \beta}^{2}$ as long as $\kappa>0$. This poses an upper bound on the mass of the singlet $m_{S_{A}}$ around $1 \mathrm{TeV}$. This bound was expected from section 2 where 
for negative $\sigma(\epsilon=-1)$ we got a strong constraint on $\Lambda_{\rho}$ which is here identified with the singlet mass up to numerical factors.

We illustrate the parameter space of the model in figure 8 . The figure also shows that the upper bound on the singlet mass we derived in eq. (3.21) gives indirectly a bound on the maximal allowed $\tan \beta$, which is even stronger constrained than in the soft Twin SUSY. An important difference between the hard $\mathbb{Z}_{2}$-breaking scenario compared and the soft one is that the masses of the MSSM Higgses controlled by $m_{A_{T}}=2 b / s_{2 \beta}$ cannot be arbitrarily decoupled if $\delta t_{\beta}$ is fixed. The $\beta$-angle misalignment in this scenario is

$$
\delta t_{\beta} \approx \frac{2}{c_{\beta}^{2} s_{2 \beta}} \frac{f^{2}}{m_{A}^{2}}\left[\left(1-\frac{2 v^{2}}{f^{2}}\right)\left(\frac{g_{\mathrm{ew}}^{2}}{8}\left|c_{2 \beta}\right|+\delta \lambda_{u} s_{\beta}^{4}\right)+\frac{\lambda_{A}^{2} \sigma_{S_{A}}\left|c_{2 \beta}\right|}{2}\right],
$$

where $\delta \lambda_{u}=\frac{3 m_{t}^{4}}{16 \pi^{2} s_{\beta}^{4} v^{4}} \log \left(\frac{M_{s}^{2}}{m_{t}^{2}} \frac{v^{2}}{f^{2}}\right)$ encodes the top-stop contribution to $\kappa$. The equation for $\tan \beta$ however stays intact up to sub-leading corrections. The expression (3.22) shows that the mass of the CP-odd Higgs is linear in $f$ and drops at lower $\tan \beta$, exactly what we see in figure 8. Note also that the range of natural values of $\delta t_{\beta}$ is of order $\mathcal{O}\left(10^{-2} \ldots 10^{-1}\right)$ and it can only be positive. Completely neglecting the $\beta$-misalignment is often not a bad approximation in this scenario.

Although light SUSY Higgses can help with the Higgs mass constraint because level splitting among CP-even Higgses can decrease the mass of the lightest eigenstate (i.e. the SM-Higgs), the masses of the SUSY Higgses are subject to bounds both from direct and indirect searches, e.g. $b \rightarrow s \gamma$ and the SM Higgs couplings fit. Even though these bounds are somewhat model dependent, we conservatively impose $m_{A} \gtrsim 400 \mathrm{GeV}$ in our numerical scan, which correspond to $m_{H^{ \pm}} \gtrsim 350 \mathrm{GeV}$ using the formulas in appendix B. We will further discuss these bounds in detail in section 4 .

At the end of the day the simplest model of Twin SUSY has a fine tuning of around $\sim 10 \%$ but is so severely constrained by $m_{h}=125 \mathrm{GeV}$ that the SUSY colored states are always forced to be within the reach of the LHC. This traces back to the fact that with a negative $\sigma$ and an irreducible tree level contribution to $\kappa$ coming from the EW D-terms, we always overshoot the Higgs mass. The only way out of this is to take $\tan \beta \sim 1$ and render the log in eq. (3.7) extremely small. The upper bound on $M_{s}$ was again expected from the discussion in section 2 when for $\epsilon=-1$ we got a strong upper bound on $\Lambda_{t}$. Interestingly, the Higgs mass constraint is acting on the parameter space of hard Twin SUSY models in the opposite direction with respect to the standard MSSM-like SUSY constructions where it often gives a lower bound on $M_{s}$.

A crucial question remains then to be addressed: Is it possible to find a model that decouples the colored states? Here we present an existence proof for such a model. Notice that in the IR effective theory for a given $\Lambda_{\rho}$ threshold one can access higher values of $\Lambda_{t}$ by introducing a negative contribution to $\kappa$ at the tree level, compensating for the unavoidable positive contribution from the D-terms. Of course this is not the only way one can explore, but we focus on this option for concreteness and comment later on possible alternative solutions. 


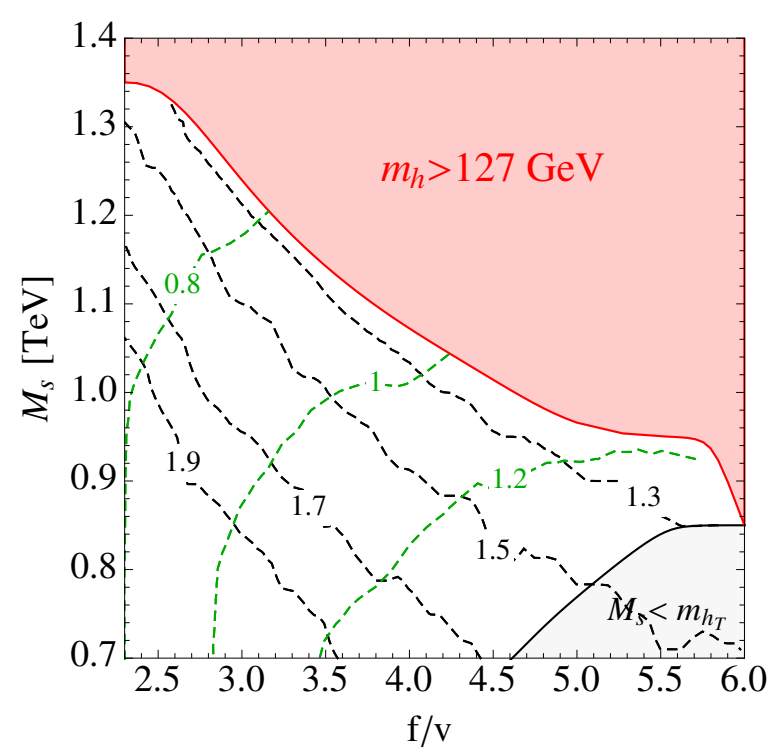

Figure 8. Allowed parameter space of the simplest hard model, described in eq. (3.18). We assume $\delta t_{\beta}=0.1$ and $\lambda_{S}=1, \mu=500 \mathrm{GeV}, M_{3}=M_{s}$. A strong upper bound on $M_{s}$ comes from the Higgs mass constraint $m_{h}<127 \mathrm{GeV}$ and the red shaded area is inaccessible. The black dashed contours stand for the maximal allowed value of $\tan \beta$ and the green dashed contours for the mass of the heaviest CP-odd Higgs in $\mathrm{TeV}$.

In order to get a negative contribution to $\kappa$ we introduce a pair of bi-doublets $B+\bar{B}$ under $\mathrm{SU}(2)_{A} \times \mathrm{SU}(2)_{B}$ with hypercharges $\pm 1 / 2$ and the following potential

$$
\Delta W_{Z_{2}}=\lambda_{B D} B h_{u}^{A} h_{u}^{B}+M_{B} B \bar{B}, \quad V_{Z_{2}}=m_{B}^{2}\left(|B|^{2}+|\bar{B}|^{2}\right) .
$$

Integrating out the bidoublet in the limit of $m_{B} \gg M_{B}, \mu$ and matching it to the Twin Higgs potential we obtain:

$$
\Delta V_{Z_{2}}=s_{\beta}^{4}\left|\lambda_{B D}\right|^{2}\left|H_{A}\right|^{2}\left|H_{B}\right|^{2} .
$$

In the Twin Higgs parametrization in eq. (2.1) this gives us a negative contribution to $\kappa$ and a positive one to $\lambda$ :

$$
\Delta \kappa=-\Delta \lambda=-\frac{1}{2} s_{\beta}^{4}\left|\lambda_{B D}\right|^{2} .
$$

Therefore we expect that for a given point in the $\left(f / v, M_{s}\right)$ plane where the $\mathbb{Z}_{2}$-breaking quartic $\lambda_{A}$ is fixed to get the desired value of $f / v$, one can always choose an appropriate $\lambda_{B}$ in order to satisfy the Higgs mass constraint, hence rescuing the hard-breaking $\mathbb{Z}_{2}$-breaking model. An analytical understanding of the structure of the EWSB conditions can be easily derived from our previous discussion performing the shift: $\delta \lambda_{u} \rightarrow \delta \lambda_{u}-\frac{\lambda_{B D}^{2}}{2}$.

Of course this solution for correcting the Higgs mass comes at a price. The main problem is the presence of states charged under both $A$ and $B$ gauge group that generically generate a mixing between the photon and the dark photon at one-loop, which is strongly constrained by experiments [47, 48]. However, this problem can be solved either by ungauging the mirror hypercharge or by breaking it, giving a mass to the mirror photon. These effects would generate extra $\mathbb{Z}_{2}$ breaking contributions in the Higgs potential which 


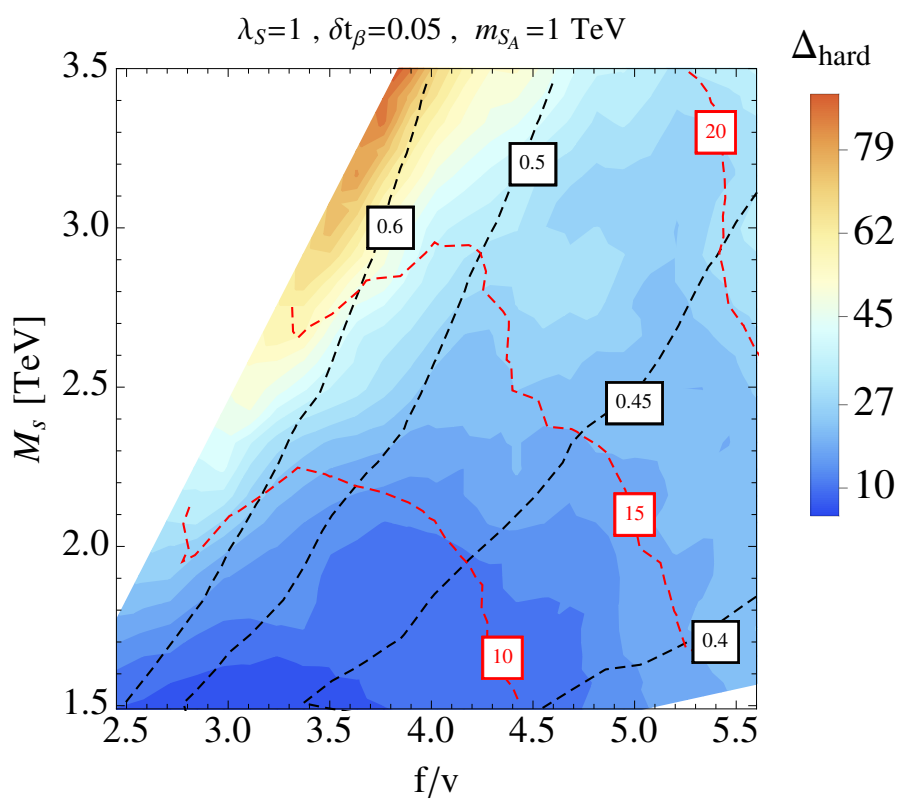

Figure 9. Allowed parameter space of hard Twin SUSY with the heavy bi-doublets. Dashed black contours stand for the minimal allowed couplings of $\lambda_{B D}$. The gradient color function indicates region of increasing fine tuning. The red dashed contours indicate the fine tuning of the EW scale with respect to $\lambda_{B D}$. We set $\delta t_{\beta}=0.05, m_{S_{A}}=1 \mathrm{TeV}$ and $m_{B}=1 \mathrm{TeV}$. In the white region a stable vacuum cannot be achieved. We also fix $\lambda_{S}=1$ and $m_{S}=1 \mathrm{TeV}, \mu=500 \mathrm{GeV}$ and $M_{3}=M_{s}$.

however do not change the structure of our model drastically. In particular the ungauging of the twin hypercharge leads to a $\mathbb{Z}_{2}$ breaking quartic via the D-terms, which however is too small to dominate the mirror symmetry breaking in a realistic scenario.

We show the parameter space of the bidoublet model in figure 9 . The bidoublet coupling $\lambda_{B D}$ ranges between $0.4 \ldots 0.6$ depending on the region of the parameter space. This corresponds to the negative shift in $\kappa$ of order $0.05 \ldots 0.1$. Among other parameters we scan here on $\tan \beta$, which however stays around $\tan \beta \lesssim 2$ in almost the entire parameter space. We calculate the fine tuning as in the previous section, varying the EW breaking scale $v$ with respect to all the parameters of the hard model. ${ }^{13}$

The behavior of the fine tuning isolines in figure 9 can be intuitively understood by means of the following approximate formula

$$
\Delta^{\text {hard }} \approx \sqrt{\left(\frac{\partial \log v^{2}}{\partial \log \lambda_{A}}\right)^{2}+\left(\frac{\partial \log v^{2}}{\partial \log \lambda_{B D}}\right)^{2}} \times \frac{\partial \log f^{2}}{\partial \log m_{H_{u}}^{2}} .
$$

The idea behind this approximation is that the fine-tuning of $f$ with respect to the SUSY scale is dominated by the running of $m_{H_{u}}$ as in soft Twin SUSY. The fine-tuning of the

\footnotetext{
${ }^{13}$ In this case the hard sector parameters include the parameters of the Higgs sector $\mathcal{P}_{H}=$ $\left\{m_{H_{u}}^{2}, m_{H_{d}}^{2}, \mu^{2}, b\right\}$, of the colored sector $\mathcal{P}_{Q}=\left\{m_{Q}^{2}, m_{U}^{2}, M_{3}\right\}$, of the $\mathrm{SU}(4)$-preserving singlet sector $\mathcal{P}_{S}=\left\{m_{S}^{2}, \lambda_{S}\right\}$ of the $\mathbb{Z}_{2}$-breaking singlet sector $\mathcal{P}_{\mathbb{Z}_{2}}=\left\{\lambda_{A}, m_{S_{A}}^{2}\right\}$ and of the bi-doublet sector $\mathcal{P}_{B}=\left\{\lambda_{B}, m_{B}^{2}\right\}$. As in the soft Twin SUSY case all these parameters are taken to be at the "messenger scale" $\Lambda_{S U S Y}$ for the purposes of the fine-tuning computation and we use full one-loop RGE (see appendix A) to obtain the final fine tuning.
} 
EW scale with respect to $f$ is instead accounted by the log-derivative with respect to the hard $\mathbb{Z}_{2}$-breaking quartic and the $\mathbb{Z}_{2}$-preserving quartic $\lambda_{B D}$ which is tuned to satisfy the Higgs mass constraint at fixed $f / v$ and $M_{s}$. This second contribution to the fine-tuning is shown on figure 9 with dashed red contours and it increases at larger $M_{s}$ as expected. The approximate formula in eq. (3.26) is the SUSY analogue of the FT measure we discussed from the low energy perspective in the right panel of figure 4. For $M_{s}=2 \mathrm{TeV}$ the theory is tuned at $\sim 3-10 \%$, depending on $f / v$ which is roughly a factor of $\sim 3-10$ better than the soft Twin SUSY model. Going to larger $M_{s}$, the fine tuning needed to satisfy $m_{h}=125 \mathrm{GeV}$ becomes larger and introducing hard $\mathbb{Z}_{2}$-breaking is not beneficial anymore. That is why we cut the parameter space of figure 9 at $M_{s} \sim 3.5 \mathrm{TeV}$.

Of course the approximate formula in eq. (3.26) relies on a number of assumptions which are not always satisfied in the full parameter space of the model. The major caveat is for sure the factorization of the fine tuning measure which is broken in the presence of extra quartics by the one-loop corrections to the soft masses proportional to both $\lambda_{A}$ and $\lambda_{B D}$ (see for example the RGE of $m_{H_{u}}$ in appendix A). These corrections are however always sub-leading compared to the top-stop contribution to the RGE of $m_{H_{u}}$ as long as both the couplings are smaller or equal to $y_{t}$ and both the singlet and the bi-doublet soft masses are not too large. For this reason we set $m_{S_{A}}=m_{B}=1 \mathrm{TeV}$ in our numerical results.

The white region at low $f / v$ region can be understood by further analyzing the expressions in eqs. (3.21) and (3.22). First of all we see from eq. (3.22) that $m_{A}$ decreases with $f / v$ and hence we expect the lower bound $m_{A}>400 \mathrm{GeV}$ to give a lower bound on $f / v$. It is then clear that small values of $\delta t_{\beta}$ are favored because they can easily raise the mass of $m_{A}$ as one can see again from eq. (3.22). In this regime we can approximate $\delta t_{\beta} \approx 0$ and solve (3.22) for $\sigma_{S_{A}}$. Plugging back this solution into (3.21) we get

$$
\lambda_{A}^{2}=-\frac{2\left(f^{2}-2 v^{2}\right)}{v^{2}}\left(\frac{\left(\delta \lambda_{u}-\frac{\lambda_{B D}^{2}}{2}\right) s_{\beta}^{2}}{\left|c_{2 \beta}\right|}+\frac{g_{\mathrm{ew}}^{2}}{4}\right),
$$

from which we see that the lower bound on $f / v$ in order to get $\lambda_{A}>0$ becomes stronger at larger $M_{s}$ (which correspond to a larger $\delta \lambda_{u}$ ), explaining the diagonal shape of the boundary of the parameter space on figure 9 .

This lower bound on $f / v$ is of course not a feature of hard Twin SUSY by itself but of our specific model. It is interesting however that it provides a lower bound on the Twin Higgs mass, which was instead bounded from above in the soft Twin SUSY model of section 3.1. Conversely the mass scale $m_{A}$ of the SUSY Higgses, which was a free parameter in the soft Twin SUSY model, is now fixed by eq. (3.22) and possibly becomes a promising signature at the LHC as we will discuss in section 4.

In conclusion, besides being sub-optimal for the reasons explained above, we believe that the SUSY model described in this section provides a simple existence proof of the hard $\mathbb{Z}_{2}$-breaking mechanism that can ameliorate the fine-tuning in perturbative UV completions of the Twin Higgs.

Of course many other constructions in the model space of hard Twin SUSY are still left unexplored. For example a possible alternative solution to the overshooting of the 
Higgs mass would be to flip the sign of the one-loop $\mathbb{Z}_{2}$-breaking threshold to $\sigma$ via a non minimal singlet sector. In such a model the constraint $m_{h}=125 \mathrm{GeV}$ can be achieved by tuning the positive $\sigma$. These solutions would be the UV completion of the effective analysis in the left panel of figure 4 and is left for future studies.

\section{Twin SUSY Higgs phenomenology}

In this section we discuss the phenomenological signatures of the SUSY Twin Higgs. In particular we focus on the Higgs sector of the theory, which leads to distinctive and model independent signatures at the LHC. The Twin Higgs mechanism implies the existence of an extra scalar in the spectrum, the "twin" Higgs, which is the radial mode of the spontaneously broken SU(4)-symmetry. The phenomenology of the twin Higgs at LHC essentially resembles the one of a scalar singlet which mixes with the SM Higgs and has been already studied e.g. in refs. [38-42]. Here we recast present and future bounds from LHC and Higgs coupling measurements on the twin Higgs focusing on the particular parameter space of Twin SUSY models (see appendix B for detailed formulas about the spectrum, the mixing angles and the decay widths). In addition we show that extra MSSM-like Higgses, which are generically present in SUSY UV completions, provide complementary probes on the parameter space of Twin SUSY both at the LHC and in indirect measurements.

We are not going to explore possible extra signatures coming from the twin matter sector. These depend very much on its structure that we leave unspecified in this paper (see refs. [27-30] for some studies about LHC signatures in Twin Higgs scenarios). Moreover, we assume pair production of colored SUSY states to be out of reach of LHC with $300 \mathrm{fb}^{-1}$, which roughly corresponds to $M_{s} \gtrsim 2 \mathrm{TeV}$. In the previous section we have shown that this regime is automatically achieved in the simplest soft Twin SUSY model once $m_{h}=125 \mathrm{GeV}$ is imposed, because the Higgs mass constraint bounds $M_{s}$ from below. For hard Twin SUSY models instead, the situation is reversed and the region with $M_{s} \gtrsim 2 \mathrm{TeV}$ is accessible at a price of some model building gymnastics.

For $M_{s} \lesssim 2 \mathrm{TeV}$ the LHC bounds on extra Higgses should be in principle compared with the canonical SUSY searches for pair produced superparticles. The same comparison should be performed for prospects at HL-LHC with $3000 \mathrm{fb}^{-1}$. We leave such a detailed comparison for future works and focus on the physics of the extra Higgses from now on.

\subsection{The Higgs sector of Twin SUSY}

The Higgs sector of the SUSY Twin Higgs exhibits a very rich structure since it contains at least a double copy of the MSSM. In section 3 we have shown that scalar states beyond the visible and hidden Higgs doublets are needed in concrete realizations of Twin SUSY in order to get the required structure of the quartics in the low energy Twin Higgs potential of eq. (2.1). Since these states are generically required to have a large soft mass of order $\sim M_{s}$, we assume that they are sufficiently heavy to be decoupled from the LHC phenomenology.

We summarize the minimal Higgs sector of Twin SUSY in table 1. The PGB formula of the SM-like Higgs mass in Twin SUSY has already been discussed in section 2, and the expressions beyond PGB can be found in appendix B. The behavior of the heavy 


\begin{tabular}{|c|c|c|c|c|}
\hline & \multicolumn{4}{|c|}{ CP-even Higgses } \\
\hline States: & $h$ & $h_{T}$ & $H$ & $H_{T}$ \\
\hline Masses: & $m_{h}^{2}$ & $\lambda_{S}^{2} s_{2 \beta}^{2} f^{2}$ & $m_{A_{T}}^{2}-\lambda_{S}^{2} f^{2}$ & $m_{A_{T}}^{2}-\lambda_{S}^{2} f^{2} s_{2 \beta}^{2}$ \\
\hline & \multicolumn{2}{|c|}{ CP-odd Higgses } & \multicolumn{2}{c|}{ Charged Higgses } \\
\hline States: & $A_{T}$ & $A$ & $H^{ \pm}$ & $H_{T}^{ \pm}$ \\
\hline Masses: & $m_{A_{T}}^{2}$ & $m_{A_{T}}^{2}-\lambda_{S}^{2} f^{2}$ & $m_{A_{T}}^{2}-\lambda_{S}^{2} f^{2}$ & $m_{A_{T}}^{2}-\lambda_{S}^{2} f^{2}$ \\
\hline
\end{tabular}

Table 1. Higgs spectrum of the Twin SUSY model.

Higgs spectrum is instead described with very good accuracy by the tree-level formulas in table 1. Here we have assumed that $\lambda_{S}^{2} \gg g_{\mathrm{ew}}^{2}, f^{2} \gg v^{2}, m_{A_{T}}^{2} \gg \lambda_{S}^{2} f^{2}$, neglected subleading corrections proportional to $\lambda_{A}$ and set $\delta t_{\beta}=0$.

From these expressions we first recognize the twin Higgs $h_{T}$ with a mass squared given by $m_{h_{T}}^{2}=4 \lambda f^{2}$, where the SU(4)-invariant quartic $\lambda$ is given by the SUSY matching condition in eq. (3.4). We further notice that among the extra Higgs states there is a fully degenerate $\mathrm{SU}(2)$-doublet $\left(H, A, H^{ \pm}\right)$with mass set by the CP-odd Higgs mass $m_{A}^{2} \equiv$ $m_{A_{T}}^{2}-\lambda_{S}^{2} f^{2}$, which becomes light at large $f$. Because this mass should be non-tachyonic, $m_{A_{T}}^{2}=2 b / s_{2 \beta}$ should always be the largest mass scale of the Higgs sector. The other SUSY $\mathrm{SU}(2)$-doublet $\left(H_{T}, A_{T}, H_{T}^{ \pm}\right)$has instead larger splittings: the heaviest state is the CP-odd Higgs $A_{T}$ with mass $m_{A_{T}}^{2}$ followed by the CP-even $H_{T}$ and the charged Higgses $H_{T}^{ \pm}$. The charged Higgses $H^{ \pm}$and $H_{T}^{ \pm}$are mass-degenerate in this approximation and do not mix because of gauge invariance. As a consequence the charged Higgses $H^{ \pm}$have exactly the same couplings as in the MSSM, while $H_{T}^{ \pm}$are completely dark and hence uninteresting for collider purposes.

The mixing of the CP-odd and the CP-even SUSY Higgses is controlled by $v / f$ exactly like the one of the SM Higgs $h$ and the twin Higgs $h_{T}: A_{T}$ and $H_{T}$ are purely dark in the limit $v / f \rightarrow 0$ while $A$ and $H$ become MSSM-like. ${ }^{14}$ The full expressions for both mixing angles and mass eigenvalues can be found in appendix B.

In most of the allowed parameter space the twin Higgs $h_{T}$ is lighter than the visible MSSM-like SU(2)-doublet and hence it is the next-to-lightest CP-even scalar after the SMlike Higgs. However, when $\lambda_{S}^{2} f^{2} \gtrsim m_{A}^{2}$ (corresponding to $m_{A_{T}}^{2} \gtrsim \lambda_{S}^{2} f^{2}$ ), the hierarchy can be inverted and a full MSSM-like SU(2)-doublet forms the first level of extra scalars above the SM-like Higgs. The transition between these two regimes is shown in figure 10, where we plot the composition of the next-to-lightest CP-even eigenstate $\mathrm{H}_{2}$ and its mass for the benchmark values of $\lambda_{S}$ and $\tan \beta$. We define the composition of mass ordered CP-even states $H_{I}$ in terms of the gauge eigenstates of the $A$ and $B$ sector as:

$$
H_{I}=\sum_{i} V_{I i} h_{i}, \quad h_{i}=\left\{h_{u}^{A}, h_{d}^{A}, h_{u}^{B}, h_{d}^{B}\right\} .
$$

In the temperature plot in figure 10 we show the combination of the mixing matrices $s_{\text {dark }} \equiv \sqrt{V_{23}^{2}+V_{24}^{2}}$ that indicate how much $H_{2}$ is composed by $B$-sector Higgses. Both

\footnotetext{
${ }^{14}$ Any mass term that mixes Higgs states in $A$ and $B$ sector must be proportional both to the breaking of $\mathrm{SU}(2)_{A}$ and $\mathrm{SU}(2)_{B}$ and therefore vanishes in the limit $v \rightarrow 0$.
} 

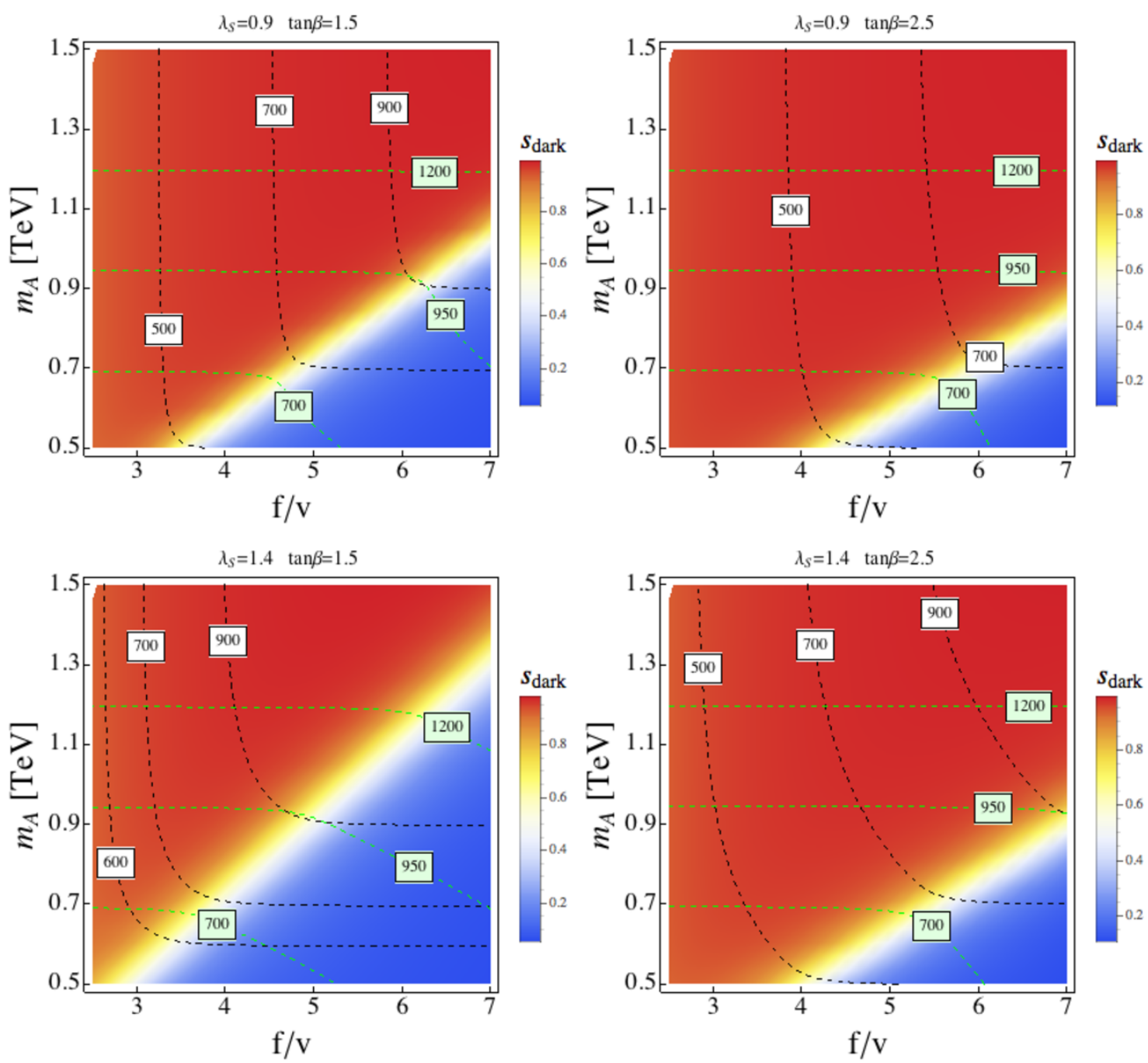

Figure 10. Mass contours in the $m_{A}$ vs $f / v$ plane. The four plots correspond to different values of $\left(\lambda_{S}, \tan \beta\right)$. The black and green contours are the second and third lightest CP-even mass state respectively. The temperature map denote the B-sector composition of the second lightest CP-even mass eigenstate as explained in the text, where $s_{\text {dark }} \equiv \sqrt{V_{23}^{2}+V_{24}^{2}}$.

mixing angles and the mass eigenvalues are computed numerically taking into account all orders in the $\lambda_{S}^{2} f^{2} / m_{A}^{2}$ expansion (setting $\lambda_{A}=\lambda_{B D}=\delta t_{\beta} \approx 0$ for simplicity).

As expected, $H_{2}$ is mainly $B$-like (i.e. $\left.h_{T}\right)$ in the upper left corner of the plot $\left(m_{A} \gtrsim\right.$ $\left.\lambda_{S} f\right)$, while it is mainly $A$-like (i.e. $\left.H\right)$ in the bottom right corner of the plot $\left(m_{A} \lesssim \lambda_{S} f\right)$. The four plots in figure 10 show how the details of the transition between the two regimes depend on the value of $\lambda_{S}$ and $\tan \beta$ : comparing the plots row-wise we see that a larger $\tan \beta$ suppresses the radial mode mass hence enlarging the parameter space in which $H_{2}$ is mostly $B$-like (red region). Column-wise, we see that a larger $\lambda_{S}$ enlarges the parameter space in which $H_{2}$ is mostly $A$-like (blue region), because the twin Higgs mass $m_{h_{T}}$ gets enhanced while the MSSM doublet mass $m_{A}$ gets suppressed. 

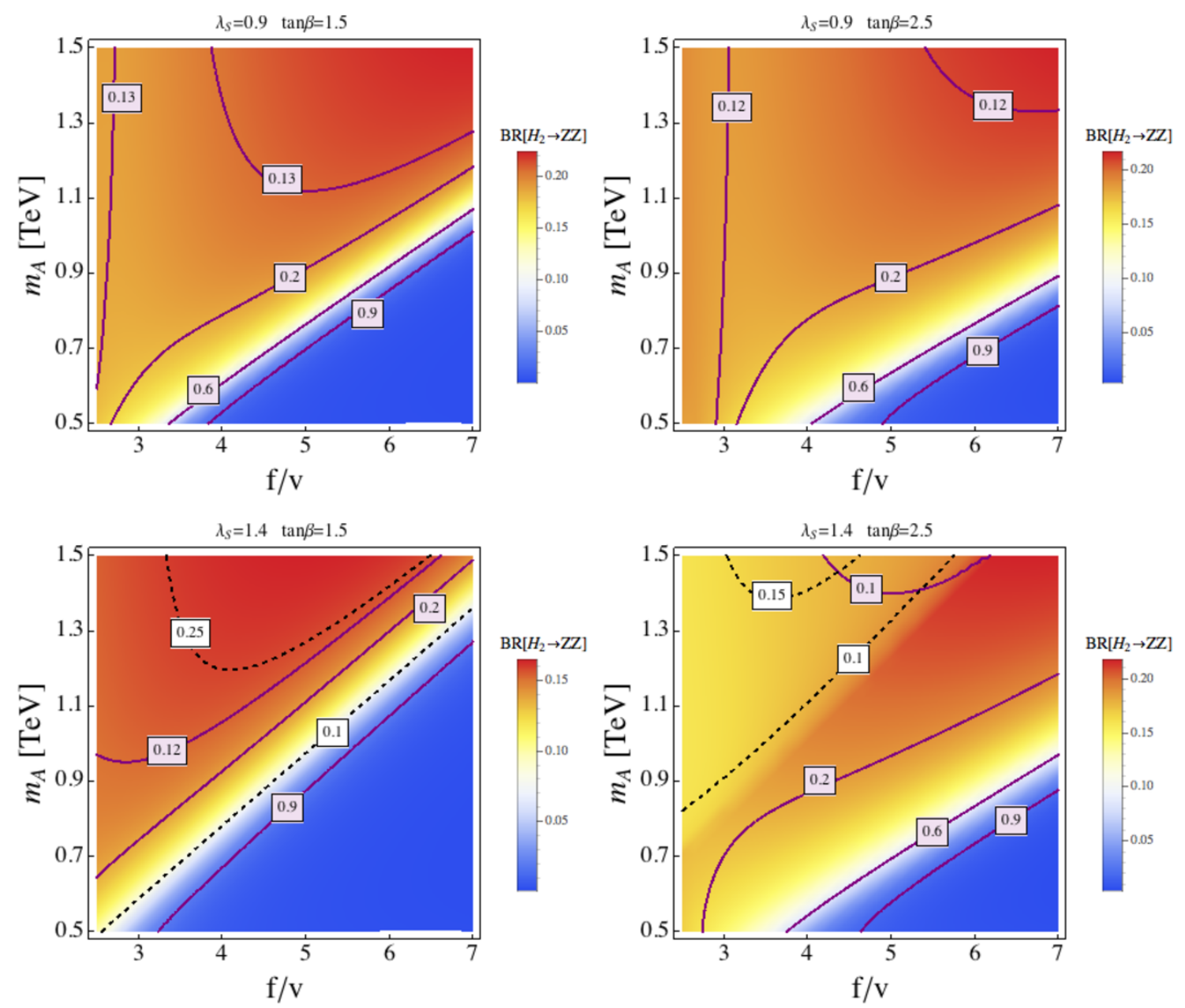

Figure 11. Branching ratio of the second lightest CP-even eigenstate. The four plots correspond to different values of $\left(\lambda_{S}, \tan \beta\right)$. The purple (black dashed) contours denotes the BR into SM $t \bar{t}$ pairs (the invisible BR into $W_{D}$ and $Z_{D}$ ). The temperature map indicates the BR into SM $Z Z$.

We are now ready to understand the parametric dependence of the decays of the CPeven Higgses in the $\left(f / v, m_{A}\right)$ plane, which we can numerically compute using the decay rates of the $\mathrm{CP}$-even eigenstates that are collected in appendix $\mathrm{B}$. In figure 11 we show the relevant branching ratios for the next-to-lightest CP-even state $\mathrm{H}_{2}$.

Clearly we have two different regions depending if the lightest CP-even state is the twin Higgs $h_{T}$ (red region) or the MSSM-like heavy Higgs $H$ (blue region). In the first case the visible decays of the twin Higgs are into pairs of SM gauge bosons/Higgses and into top pairs. An invisible decay width can also be present if the decays into dark gauge bosons are kinematically open. Since we assume equal gauge couplings in the visible and dark sector, the dark gauge boson masses are fixed and scale approximately linearly with $f$, e.g. $m_{W_{B}} \approx \frac{g_{2}}{\sqrt{2}} f$. Comparing the upper and the lower panel of figure 11 we see that a non-zero invisible decay width for the twin Higgs ultimately depends on the value of the SU(4)-invariant coupling $\lambda_{S}$, which sets the scale of the twin Higgs mass. In the upper row we take $\lambda_{S}=0.9$, such that the invisible decay channels are kinematically closed while 
in the second row we take $\lambda_{S}=1.4$. Of course the latter value is favored by fine-tuning arguments as discussed in section 3 , however since $\lambda_{S} \lesssim 1$ makes the phenomenology of the twin Higgs radically different we decided to include $\lambda_{S}=0.9$ in our discussion.

The most interesting decay channel to hunt for the twin Higgs at the LHC is certainly the one into $Z$ pairs. In figure 11 we see that this branching ratio goes roughly from $25 \%$ to $10 \%$ in the red area, depending on the region of the parameter space. A $25 \%$ branching ratio into $Z$ pairs is the value expected from the Goldstone equivalence principle if one assumes $\sim 100 \%$ branching ratio of the twin Higgs into SM gauge bosons/Higgs like was done in [38]. From figure 11 we see that this expectation is only (marginally) saturated in the region where $m_{A}>1 \mathrm{TeV}$ and $f / v>5$. In the bulk of the SUSY Twin Higgs parameter space the branching ratio into $Z$ pairs (like the ones into $W W$ and $h h$ ) is depleted because of an irreducible branching ratio into top pairs (purple contours). The $t \bar{t}$ channel is suppressed only for very large $f / v$ (i.e. very small mixing) or for very small $f / v$ because of the reduced phase space.

Using the formulas from appendix $\mathrm{B}$, assuming $m_{A} \gg \lambda_{S} f$ and neglecting the phase space suppressions we get

$$
\frac{\Gamma\left(h_{T} \rightarrow Z Z\right)}{\Gamma\left(h_{T} \rightarrow t \bar{t}\right)} \approx \frac{m_{h_{T}}^{2}}{12 c_{W}^{2} m_{t}^{2}} \approx \frac{\lambda_{S}^{2} s_{2 \beta}^{2} f^{2}}{12 c_{W}^{2} m_{t}^{2}} \approx 1 \times\left(\frac{f / v}{3}\right)^{2} \times\left(\frac{\lambda_{S}}{1}\right)^{2} \times\left(\frac{s_{2 \beta}}{0.98}\right)^{2},
$$

which shows that an irreducible branching ratio into $t \bar{t}$ for the twin Higgs is somewhat typical for Twin SUSY scenarios, where the branching ratio into $Z Z$ gets suppressed for $\tan \beta>1$ (where $\sin 2 \beta$ is reduced) and cannot be enhanced arbitrarily by taking a larger $\lambda_{S}$ because of perturbativity.

In the second row of figure 11 we set $\lambda_{S}=1.4$ and the invisible width of the twin Higgs is different from zero (dashed black contours). When the decays into dark gauge bosons is kinematically open, if one would assume $100 \%$ decay into (Pseudo)-Goldstones, the Goldstone equivalence theorem would predict a branching ratio of $\sim 0.14$ into $Z Z$ and an invisible width of $\sim 3 \times 0.14$. However, this naive estimate is once again modified by the irreducible decay width into $t \bar{t}$ (though less relevant than $\lambda_{S}=0.9$ in agreement with formula (4.2)) and by the fact that in most of the Twin SUSY parameter space the twin Higgs mass is not parametrically larger than the dark gauge boson masses. Indeed the invisible width of the radial mode gets sensibly reduced because of phase space suppression, as can be seen from the contours in figure 11. The phase space suppression of the invisible channels becomes more important at larger $\tan \beta$, where the twin Higgs mass is further reduced.

Finally, for fixed value of $f / v$, decreasing the value of $m_{A}$ implies that the mixing with the MSSM-like CP-even Higgs becomes important and the branching ratio into $t \bar{t}$ is enhanced with respect to $Z Z$ (and with respect to invisible ones if present). Decreasing $m_{A}$ further the MSSM-like Higgs becomes the next-to-lightest state and as a consequence the decay width into $Z Z$ is suppressed while the one into $t \bar{t}$ gets rapidly close to $100 \%$.

\subsection{Probing the SUSY Twin Higgses}

From the previous discussion one can infer what are the most promising phenomenological signatures associated to the Higgs sector of the SUSY Twin model. We essentially have 
two different types of signatures depending on the region of the parameter space:

- In the region where $m_{A} \gtrsim \lambda_{S} f$ the twin Higgs is the next-to-lightest CP-even state (this is the red region in figures 10 and 11). The radial mode is copiously produced at the LHC via its mixing with the SM Higgs and the most promising channels to probe it are di-boson final states; in particular di-Higgs (four b-jets) or Z-boson pairs $[20,38,43,49]$. Indirect bounds on the twin Higgs also arise from modifications of the SM-like Higgs couplings (in particular the ones to gauge bosons).

- In the region where $m_{A} \lesssim \lambda_{S} f$ we have a full MSSM-like $\mathrm{SU}(2)$ doublet which becomes light. Since having $m_{h}=125 \mathrm{GeV}$ always forces $\tan \beta$ to be quite small, this region of the parameter space is preferably tested by searches for charged MSSM-like Higgses. These can be probed either indirectly through their contribution to $b \rightarrow s \gamma$, or directly hunted at the LHC via $t \bar{b}$ and $b \bar{t}$ final states $[43,50]$. Another interesting channel is provided by the associated production of a CP-even/odd Higgs with $t \bar{t}$ [43]. However, searches for charged MSSM-like Higgses typically provide a cleaner channel for similar mass scales.

We now provide a preliminary study of the LHC reach on the parameter space of Twin SUSY models, which focus on direct and indirect searches for the twin Higgs and charged MSSM-like Higgses. We present both existing constraints and the future reach of LHC searches and indirect measurements. For LHC prospects we consider projections for the final stage of LHC with $300 \mathrm{fb}^{-1}$ and for HL-LHC, where $3000 \mathrm{fb}^{-1}$ are expected [51].

For the $Z Z$ channel we use the results of ref. [38], where the current and future bounds for a scalar singlet mixing with the SM-Higgs are given as a function of its mass and signal strength into $Z Z$. We recast these bounds in the Twin SUSY parameter space by computing the production cross section of the radial mode from both gluon fusion and vector boson fusion $(\mathrm{VBF})$ processes and multiplying by the branching ratio into $Z Z{ }^{15}$

For what concerns Higgs coupling deviations, a global fit of Higgs couplings and EWPT in Twin SUSY has already been performed in ref. [20], where it has been shown that current data cannot probe our region of interest on which we already impose $f / v \gtrsim 2.3$ and $m_{A} \gtrsim 400 \mathrm{GeV}$. To estimate the HL-LHC prospects on Higgs couplings, one can focus on the measurement of the SM Higgs coupling to $Z$-bosons. The HL-LHC prospects for the Higgs coupling measurements will ameliorate the current bound on the mixing angle by roughly a factor of 2 (the precise numbers can be found in refs. [38] and [53]), which leads to a future bound of approximately $f / v \gtrsim 4.6$. We will comment on such a limit in the following discussion.

The LHC prospects for direct probe of the MSSM charged Higgses are taken from ref. [43] where the process $p p \rightarrow H^{+} \bar{t} b \rightarrow t \bar{b} \bar{t} b$ (and charged conjugate) is employed. Notice that we can use these bounds without any further modification since the visible charged Higgses do not mix with the dark sector ones.

\footnotetext{
${ }^{15}$ To estimate the $8 \mathrm{TeV}$ cross section we use the Higgs production cross section for the gluon fusion and the VBF processes for equivalent mass [52] and weight them with the appropriate mixing angles. For the $\sqrt{s}=13 \mathrm{TeV}$ and $\sqrt{s}=14 \mathrm{TeV}$ cross section we just re-weight the $\sqrt{s}=8 \mathrm{TeV}$ cross sections for gluon fusion and VBF with the corresponding parton luminosities ratios taken from ref. [52].
} 
Concerning the indirect searches through $b \rightarrow s \gamma$, we parametrize the BSM contributions as modifications to the Wilson coefficients $\Delta C_{7}$ and $\Delta C_{8}$ with respect to their SM values [54]:

$$
B_{s \gamma}^{\text {th }} \times 10^{4}=3.36 \pm 0.23-8.22 \Delta C_{7}-1.99 \Delta C_{8},
$$

where \pm 0.23 is the theoretical uncertainty of the Standard Model prediction. For the experimental measurement we use the combined result from ref. [55]

$$
B_{s \gamma}^{\exp } \times 10^{4}=3.41 \pm 0.16
$$

and we derive the bounds allowing for $2 \sigma$ deviations from the experimental value, summing in quadrature the theoretical and experimental uncertainties. The contribution of the charged MSSM-like Higgs to this observable implies a lower bound on its mass for a fixed value of $\tan \beta$. In particular, the existing limits set a lower bound of around $m_{H^{+}} \gtrsim$ $500 \mathrm{GeV}$ for $\tan \beta \simeq 2$, and hence does not constraint our parameter space. In order to estimate the future prospects for this observable, we optimistically assume that both the theoretical and the experimental uncertainties get reduced by a factor of $2 .{ }^{16}$ The bound on the charged MSSM-like Higgs mass is then significantly improved and goes up to $m_{H^{+}} \geq 775 \mathrm{GeV}$ for $\tan \beta \simeq 2$.

In figure 12 we project the current and future bounds from the searches described above in the $\left(f / v, m_{A}\right)$ plane. Analogously to figures 10 and 11 we show the results for four different choices of $\lambda_{S}=\{1.4,0.9\}$ and $\tan \beta=\{1.5,2.5\}$. From these plots we can see that direct searches for the twin Higgs in the $Z Z$ channel give a lower bound on $f / v$ as long as $m_{A} \gtrsim \lambda_{S} f$. In contrast direct and indirect searches on the MSSM charged Higgses set a lower bound on $m_{A}$ independently on the value of $f$. We first discuss the features of the searches targeting the MSSM-like scalars. Comparing the plots row-wise we see that direct LHC searches on the charged Higgs depend significantly on $\tan \beta$, which sets the couplings to $\bar{t} b$. The projected bound from $b \rightarrow s \gamma$ is also dependent on $\tan \beta$ and gets slightly stronger for very low values of $\tan \beta$. Note that here we used the complete expression for the charged Higgs mass which includes also $\mathcal{O}\left(v^{2}\right)$ corrections. Conversely, comparing plots column-wise one can see that varying $\lambda_{S}$ does not change the physics of the MSSM charged Higgs as expected.

Now we focus on the region probed by the di-boson resonance searches. The shape of the exclusion lines from the $Z Z$ final states presents a rich structure, which depends on multiple effects controlled by $\lambda_{S}$ and $\tan \beta$. The overall reach is affected by variations of the coupling to tops (the main production mechanism is gluon fusion), variations in the structure of the mass spectrum (cf. figure 10) and in the branching ratio to $Z Z$ (cf. figure 11). However, independently on the values of $\lambda_{S}$ and $\tan \beta$, the exclusion regions exhibit a peculiar horn-like shape. The horn is located where the next-to-lightest eigenstate changes from mostly twin-like to mostly MSSM-like In such region, because of the transition, there is a local enhancement in the A-sector components of the $H_{2}$ state and the sensitivity is increased. Then, in the region where the $\mathrm{H}_{2}$ state is very much MSSM-like, the sensitivity drops because of the reduced branching ratio into gauge bosons. In the low $m_{A}$ region of

\footnotetext{
${ }^{16} \mathrm{M}$. Misiak private communication.
} 

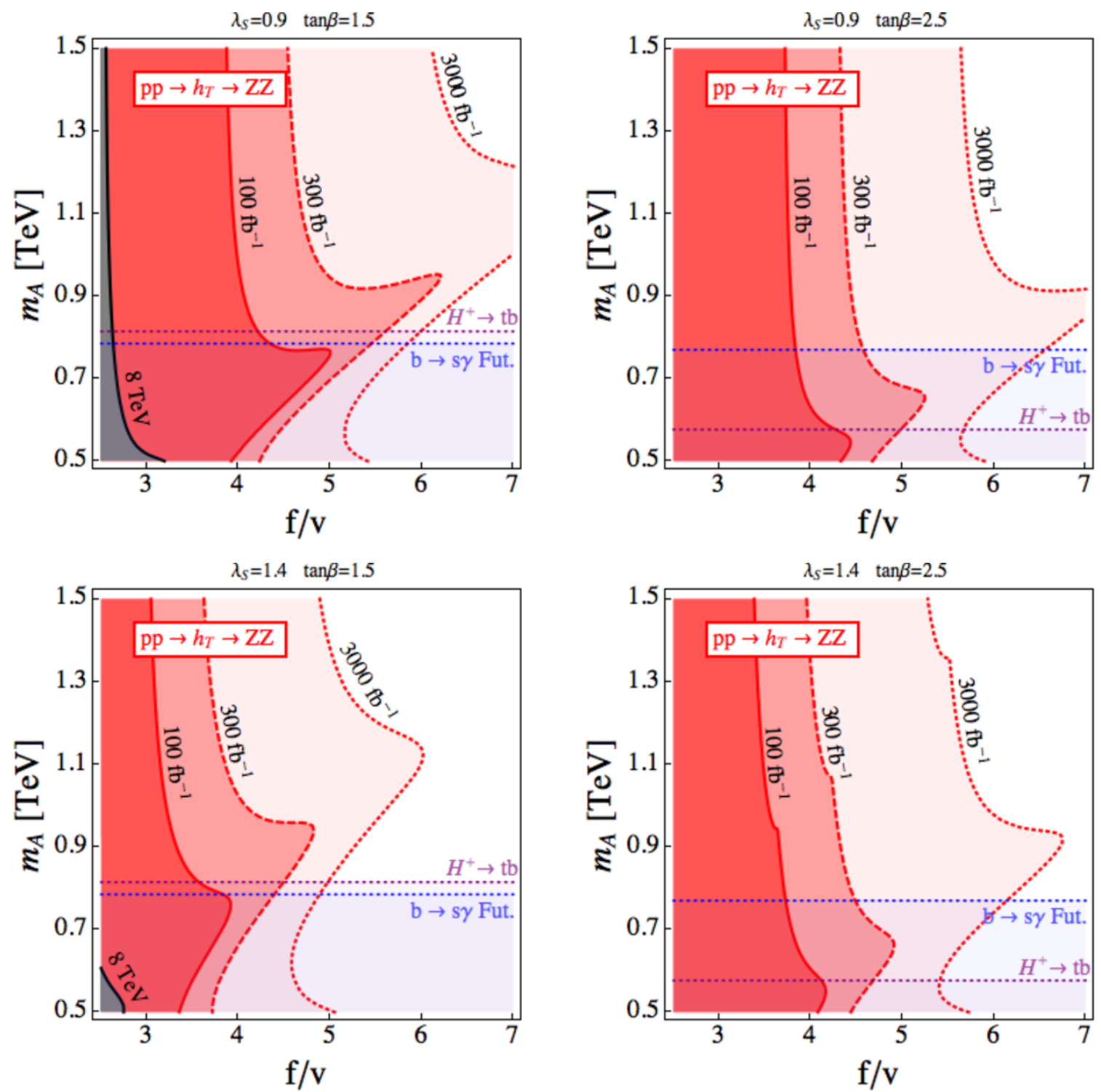

Figure 12. Phenomenology of the SUSY Twin Higgs model in the $\left(f / v, m_{A}\right)$ plane. The dark grey region denotes the $8 \mathrm{TeV}$ exclusion from di-boson searches. The red regions denote the LHC prospects for di-boson signatures associated with the twin Higgs state, respectively with 100, 300 and $3000 \mathrm{fb}^{-1}$ for the solid contour, the dashed contour and the dotted contour. Projected bounds from $b \rightarrow s \gamma$ are denoted in blue while the HL-LHC reach on the charged Higgs state with $3000 \mathrm{fb}^{-1}$ is indicated in purple. 
the plots (close to $m_{A} \sim 500 \mathrm{GeV}$ ) the reach of HL-LHC is again enhanced because the very small branching ratio of $H_{2}$ into gauge bosons is compensated by a sizeable enhancement of the production cross section for such low masses.

In the first row of figure 12 we fix $\lambda_{S}=0.9$ such that the invisible decay channels are closed. Comparing the plots row-wise we see that the bounds from LHC are very similar. The reason is that for lower $\tan \beta$ the enhancement of the top coupling, and hence of the gluon fusion channel, is partially compensated by a larger twin Higgs mass (cf. the contours of figure 10). The transition between the twin Higgs regime and the MSSM-like regime is not the same in the two plots and reflects the discussion of the previous subsection.

In the second row of figure 12 we fix $\lambda_{S}=1.4$ and the invisible decay channel for the twin Higgs opens up, depleting the signal strength into $Z Z$ with respect to the $\lambda_{S}=0.9$ case. However, in the bottom right plot the larger value of $\tan \beta$ reduces $m_{h_{T}}$ and closes the invisible decay into dark gauge bosons exactly where the small bump in the exclusion curves is, roughly where $m_{h_{T}} \sim 2 m_{W_{D}}$ (compare with contours in figure 10).

In summary, we see that searches for the twin Higgs into $Z Z$ final states at the LHC are extremely interesting in Twin SUSY constructions, because perturbativity of the SU(4)invariant coupling $\lambda_{S}$ and $\tan \beta>1$ give an upper bound on the twin Higgs mass for fixed value of $f / v$ and fixed $m_{A}$. This has to be contrasted with strongly coupled UV completions where usually the strongest constraint comes from Higgs coupling measurements. Note that the prospects for Higgs coupling measurements at HL-LHC reach roughly the value of $f / v \simeq 4.6$ as we mentioned before. For the largest $\lambda_{S}$ we considered, which corresponds to the largest $m_{h_{T}}$ compatible with perturbativity, the Twin SUSY phenomenology starts to be similar to strongly coupled UV completions with the Higgs coupling measurements having a reach not very far from the direct searches (see left bottom plot of figure 12). However, for lower values of $\lambda_{S}$ Higgs coupling measurements are never competitive with the direct resonance search as can be seen from the other plots on figure 12. Finally, the region with large $f / v$ features a radial mode too heavy to be probed in $Z Z$ searches at the LHC, even at High Luminosity. For small $m_{A}$, the spectrum here resembles the usual MSSM and MSSM-like Higgses searches will explore this region for $m_{A} \lesssim 1 \mathrm{TeV}$.

Looking back at the parameter space of the soft and the hard Twin SUSY models of section 3, we can roughly compare the sensitivity of the direct searches at the LHC in these two particular cases. In the simplest soft Twin SUSY model of figure 5 and figure 7 we found a strong upper bound on $f / v$ for $M_{s} \approx 2 \mathrm{TeV}$, which, together with the quite large value of $\tan \beta \approx 3.5$, gives a stringent upper bound on the mass of the twin Higgs $\lesssim 400-700 \mathrm{GeV}$, depending on the particular choice of $\delta t_{\beta}$ and $m_{A_{T}}$. A considerable amount of parameter space of soft Twin SUSY is then likely to be probed by $Z Z$ searches already with $100 \mathrm{fb}^{-1}$ of data. The mass of the MSSM-like Higgses depends instead on $m_{A_{T}}$, which is unconstrained in this model and can always be taken to be heavy.

The hard Twin SUSY model we presented in figure 9 has instead a lower bound on the Twin Higgs mass which increases at large $M_{s}$. This lower bound is still pretty mild for $M_{s} \approx 2 \mathrm{TeV}$ and Twin Higgs searches are certainly promising to explore these models. Quite interestingly the masses of the MSSM-like Higgses cannot be arbitrarily decoupled in this model as far as natural values of $\delta t_{\beta}$ are considered and the charged Higgses can 
easily be below the $\mathrm{TeV}$ leaving some hope for indirect signatures in $b \rightarrow s \gamma$ or the direct searches at the LHC.

\section{Conclusions}

In this work we have performed a systematic study of perturbative UV completions of the Twin Higgs mechanism based on Supersymmetry. In this context we showed that breaking the $\mathbb{Z}_{2}$ mirror symmetry with large quartics can be beneficial in terms of fine tuning, leading to theories with colored states decoupled from the LHC and tuned to the level of $\sim 10 \%$.

In order to explore the role of $\mathbb{Z}_{2}$-breaking quartics, we performed a detailed comparative study between hard and soft breaking of the $\mathbb{Z}_{2}$-symmetry from the effective field theory point of view in section 2. This study provides a complete picture of the parameter space of the Twin Higgs and opens up new model building avenues. The main result is that hard $\mathbb{Z}_{2}$-breaking models can lead to a gain in fine tuning with respect to soft $\mathbb{Z}_{2}$-breaking at the price increasing the SM-like Higgs mass, which is usually predicted to be too high. Once the Higgs mass constraint is satisfied, the fine tuning of hard $\mathbb{Z}_{2}$-breaking models can still be around a factor of $\sim 5$ better compared to soft $\mathbb{Z}_{2}$-breaking.

We have studied explicit SUSY UV completions of both soft and hard $\mathbb{Z}_{2}$-breaking models. We performed our analysis both numerically, solving directly the EWSB conditions and the Higgs mass constraint in the UV theory, and analytically, using a simple tree level matching to the original Twin Higgs model after the SUSY states are decoupled. Both models are not saturating the parametric gain in fine-tuning that we naïvely expect from Twin SUSY theories with respect to standard SUSY scenarios, but provide simple existence proofs of both mechanisms of mirror symmetry breaking. By comparing these two simple SUSY models we recover the gain in fine tuning of the hard $\mathbb{Z}_{2}$-breaking model as obtained in the effective field theory. As a summary plot we show in figure 13 the allowed FT range in the soft and the hard model as a function of the scale of the colored particles, marginalized over the rest of the parameters.

The Twin SUSY constructions discussed here are only valid up to a scale of roughly $\sim 100 \mathrm{TeV}$. The final goal would be to build full UV completions up to the GUT scale where both the SUSY-breaking and the $\mathbb{Z}_{2}$-breaking are generated dynamically and then mediated to the MSSM and its mirror copy. Specifying the mechanism of mediation will give a definite prediction on the SUSY spectrum of both the visible and the mirror sector. Further studies in this direction are definitely worthwhile.

Our analysis has unveiled several important aspects of the parameter space of Twin SUSY models, highlighting in particular the special role of the Higgs mass in constraining the parameter space of both soft and hard Twin SUSY. To improve the analysis it would be desirable to have a more precise computation of the SM Higgs mass including at least 2-loop QCD corrections. The implementation of Twin SUSY models in a package like SARAH [56] can bring them to a similar precision we have for standard SUSY scenarios. When all the SUSY states are heavy enough one can use effective potential techniques matching to the non SUSY Twin Higgs potential in (2.1). For this potential the higher loop contributions have been computed at NNLL in [57]. 


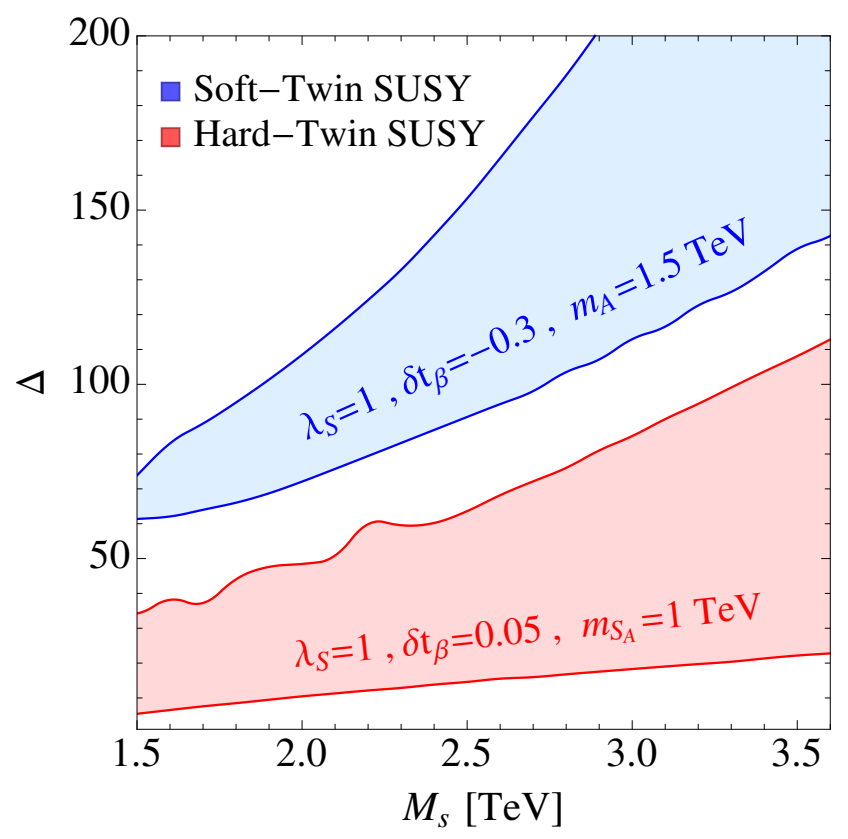

Figure 13. Comparison of the fine tuning of the soft Twin SUSY and hard one. Inside the strips we marginalize over all the parameters of the model unless specified.

We hope that our study elucidated the basic building blocks of Twin SUSY constructions and their connection to the original Twin Higgs proposal. With these tools at hand it will be easier to construct "optimal" models of Twin SUSY, in attempt of maximizing their parametric gain in fine tuning with respect to standard SUSY scenarios.

We also sketched the phenomenology of the Twin SUSY Higgses.

In particular we showed that LHC searches for a resonance going into di-bosons can probe a large portion of the parameter space of Twin Higgs models, independently on how the mirror symmetry is broken. This is a consequence of the requirement of perturbativity on the $\mathrm{SU}(4)$-invariant quartic coupling which gives an upper bound on the mass of the twin Higgs at fixed SU(4)-breaking VEV $f$, and it is a distinctive feature of perturbative UV completions of the Twin Higgs mechanism.

It would be interesting to further characterize the signatures associated to the invisible decays of the twin Higgs. These can potentially distinguish it from a generic singlet that mixes with the SM Higgs. Indeed, the invisible branching ratio of the twin Higgs is likely to be enhanced when extra light SUSY states are present in the mirror sector making it discoverable at the LHC in final states with large missing transverse energy (MET).

Interesting signatures can also arise from MSSM-like Higgses which become light at large $f$. Twin SUSY models then provide an additional motivation to extend the search program for extra MSSM-like Higgses in the small $\tan \beta$ regime both at the LHC and at future colliders $[43,58-60]$. It would be interesting to investigate in detail alternative final states to probe CP-odd and CP-even MSSM-like scalars, such as the ones suggested in refs. $[43,59]$ including for instance $t \bar{t} A / H$ production. The possibility of having extra invisible decay channels into the mirror sector can also give rise to final states with MET, enriching further the landscape of accessible topologies. 
The phenomenology of the extended Higgs sector is just the tip of an iceberg of possible phenomenological questions one can ask in the context of Twin SUSY. By specifying the detailed structure of both the SUSY and the mirror spectrum one can explore further the phenomenological implications of Twin SUSY. For instance it would be interesting to investigate allowed cosmological scenarios as it has been done for the original Twin Higgs proposal [32-37], identifying possible dark matter candidates (both symmetric and asymmetric), viable mechanisms for baryogenesis etc. Of course extra LHC signatures other than the ones associated to extra Higgses can arise once the full spectrum is specified (see for example refs. [27-30]) and might require interesting new detecting techniques [61, 62]. All these instances are likely to have an impact on the fine tuning of Twin SUSY models establishing a unique interplay between cosmology, collider searches and naturalness which is a distinctive features of $\mathrm{NN}$ scenarios.

\section{Acknowledgments}

We are grateful to Nathaniel Craig, Mark Goodsell, Matthew McCullough, Mikolaj Misiak, Filippo Sala, Pietro Slavich and Riccardo Torre for useful discussions and Lorenzo Ubaldi for comments on the manuscript. The work of A.K. was performed in part at the Aspen Center for Physics, which is supported by National Science Foundation grant PHY1066293. The work of D.R. and R.Z. was mostly performed at the Laboratoire de Physique of Hautes Energies (LPTHE) in Paris. D.R. R.Z. and A.M. also acknowledge the hospitality of the Galileo Galilei Institute for Theoretical Physics. A.M. is supported by the Strategic Research Program High Energy Physics and the Research Council of the Vrije Universiteit Brussel. A.M. is also supported in part by the Belgian Federal Science Policy Office through the Interuniversity Attraction Pole P7/37. The work of SP is supported by the National Science Centre, Poland, under research grants DEC-2014/15/B/ST2/02157, DEC-2012/04/A/ST2/00099 and DEC-2015/18/M/ST2/00054.

\section{A Renormalization Group Equations}

In this appendix we provide the one-loop RGEs for the Twin SUSY models with a) soft $\mathbb{Z}_{2}$-breaking, b) hard $\mathbb{Z}_{2}$-breaking and c) hard $\mathbb{Z}_{2}$-breaking with bi-doublets. We neglect A-terms, singlet B-terms, electro-weakinos, sbottom and slepton masses, and take into account three families both in the visible and the dark sector (relevant for gauge coupling running and hypercharge D-terms).

\section{A.1 Soft $\mathbb{Z}_{2}$-breaking model}

Superpotential and soft terms. The superpotential (in the UV) is defined as

$$
\left.W\right|_{\mathrm{UV}}=\left(\mu+\lambda_{S} S\right)\left(h_{u}^{A} h_{d}^{A}+h_{u}^{B} h_{d}^{B}\right)+y_{t}\left(Q_{3}^{A} U_{3}^{A} h_{u}^{A}+Q_{3}^{B} U_{3}^{B} h_{u}^{B}\right)+\frac{1}{2} M_{S} S^{2},
$$


and soft breaking masses (in the UV) are given by

$$
\begin{aligned}
\left.V_{\mathrm{soft}}\right|_{\mathrm{UV}}= & m_{H_{u}}^{2}\left(\left|h_{u}^{A}\right|^{2}+\left|h_{u}^{B}\right|^{2}\right)+m_{H_{d}}^{2}\left(\left|h_{d}^{A}\right|^{2}+\left|h_{d}^{B}\right|^{2}\right)-b\left(h_{u}^{A} h_{d}^{A}+h_{u}^{B} h_{d}^{B}+\text { h.c. }\right) \\
& +m_{Q}^{2}\left(\left|Q_{3}^{A}\right|^{2}+\left|Q_{3}^{B}\right|^{2}\right)+m_{U}^{2}\left(\left|U_{3}^{A}\right|^{2}+\left|U_{3}^{B}\right|^{2}\right)+m_{S}^{2}|S|^{2} \\
& +\Delta m_{H_{u}}^{2}\left(\left|h_{u}^{A}\right|^{2}-\left|h_{u}^{B}\right|^{2}\right)+\Delta m_{H_{d}}^{2}\left(\left|h_{d}^{A}\right|^{2}-\left|h_{d}^{B}\right|^{2}\right) .
\end{aligned}
$$

Note that $\mathbb{Z}_{2}$ is broken in the UV Lagrangian only by soft Higgs masses, which implies that $\mathbb{Z}_{2}$ is conserved by the RG flow except for sfermion (stop) masses. Therefore we have to add $\mathbb{Z}_{2}$-odd stop masses in the IR-potential for consistency (but they are negligible since the $\mathbb{Z}_{2}$-breaking Higgs masses in the UV are taken to be small in the first place)

$$
\left.\delta V_{\text {soft }}\right|_{\mathrm{IR}}=\Delta m_{Q}^{2}\left(\left|Q_{3}^{A}\right|^{2}-\left|Q_{3}^{B}\right|^{2}\right)+\Delta m_{U}^{2}\left(\left|U_{3}^{A}\right|^{2}-\left|U_{3}^{B}\right|^{2}\right) .
$$

Beta functions. The beta function coefficients for the gauge couplings $\tilde{g}_{Y}=g_{Y}, \tilde{g}_{2}=$ $g_{2}, \tilde{g}_{3}=g_{3}$ are:

$$
b_{i}=(11,1,-3) \text {. }
$$

\section{Yukawa couplings}

$$
\begin{aligned}
16 \pi^{2} \frac{d}{d t} \lambda_{S} & =\lambda_{S}\left(6 \lambda_{S}^{2}+3 y_{t}^{2}-g_{Y}^{2}-3 g_{2}^{2}\right) \\
16 \pi^{2} \frac{d}{d t} y_{t} & =y_{t}\left(6 y_{t}^{2}+\lambda_{S}^{2}-\frac{13}{9} g_{Y}^{2}-3 g_{2}^{2}-\frac{16}{3} g_{3}^{2}\right) .
\end{aligned}
$$

\section{SUSY masses}

$$
\begin{aligned}
16 \pi^{2} \frac{d}{d t} \mu & =\mu\left(2 \lambda_{S}^{2}+3 y_{t}^{2}-g_{Y}^{2}-3 g_{2}^{2}\right), \\
16 \pi^{2} \frac{d}{d t} M_{S} & =8 M_{S} \lambda_{S}^{2} .
\end{aligned}
$$

Soft masses. Neglecting the contributions of $\mathbb{Z}_{2}$-breaking stop masses to the RGEs, one has

$$
\begin{aligned}
16 \pi^{2} \frac{d}{d t} m_{H_{u}}^{2} & =g_{Y}^{2} \xi+2 \lambda_{S}^{2} X_{S}+6 y_{t}^{2} X_{t}, \\
16 \pi^{2} \frac{d}{d t} \Delta m_{H_{u}}^{2} & =g_{Y}^{2} \Delta \xi+2 \lambda_{S}^{2} \Delta X_{S}+6 y_{t}^{2} \Delta X_{t}, \\
16 \pi^{2} \frac{d}{d t} m_{H_{d}}^{2} & =-g_{Y}^{2} \xi+2 \lambda_{S}^{2} X_{S}, \\
16 \pi^{2} \frac{d}{d t} \Delta m_{H_{d}}^{2} & =-g_{Y}^{2} \Delta \xi+2 \lambda_{S}^{2} \Delta X_{S}, \\
16 \pi^{2} \frac{d}{d t} m_{Q}^{2} & =\frac{1}{3} g_{Y}^{2} \xi-\frac{32}{3} g_{3}^{2} M_{3}^{2}+2 y_{t}^{2} X_{t}, \\
16 \pi^{2} \frac{d}{d t} m_{U}^{2} & =-\frac{4}{3} g_{Y}^{2} \xi-\frac{32}{3} g_{3}^{2} M_{3}^{2}+4 y_{t}^{2} X_{t}, \\
16 \pi^{2} \frac{d}{d t} m_{S}^{2} & =8 \lambda_{S}^{2} X_{S},
\end{aligned}
$$


with the auxiliary functions

$$
\begin{aligned}
X_{S} & \equiv m_{S}^{2}+m_{H_{u}}^{2}+m_{H_{d}}^{2}, & \Delta X_{S} & \equiv \Delta m_{H_{u}}^{2}+\Delta m_{H_{d}}^{2}, \\
X_{t} & \equiv m_{Q}^{2}+m_{U}^{2}+m_{H_{u}}^{2}, & \Delta X_{t} & \approx \Delta m_{H_{u}}^{2}, \\
\xi & \equiv 3 m_{Q}^{2}-6 m_{U}^{2}+m_{H_{u}}^{2}-m_{H_{d}}^{2}, & \Delta \xi & \approx \Delta m_{H_{u}}^{2}-\Delta m_{H_{d}}^{2} .
\end{aligned}
$$

The RGEs for $\mathbb{Z}_{2}$-breaking stop masses are given by

$$
\begin{aligned}
16 \pi^{2} \frac{d}{d t} \Delta m_{Q}^{2} & =\frac{1}{3} g_{Y}^{2} \Delta \xi+2 y_{t}^{2} \Delta X_{t}, \\
16 \pi^{2} \frac{d}{d t} \Delta m_{U}^{2} & =-\frac{4}{3} g_{Y}^{2} \Delta \xi+4 y_{t}^{2} \Delta X_{t} .
\end{aligned}
$$

Moreover one has

$$
\begin{aligned}
16 \pi^{2} \frac{d}{d t} b & =b\left(10 \lambda_{S}^{2}+3 y_{t}^{2}-3 g_{2}^{2}-g_{Y}^{2}\right), \\
16 \pi^{2} \frac{d}{d t} M_{3} & =-6 g_{3}^{2} M_{3} .
\end{aligned}
$$

\section{A.2 Hard $\mathbb{Z}_{2}$-breaking + bi-doublets}

For the sake of brevity we give the RGEs only for the general model with bi-doublets. The RGEs of the hard breaking model are obtained by setting to zero the bi-doublet parameters $\lambda_{B D}, M_{\Phi}, m_{B}^{2}, m_{\bar{B}}^{2}$.

Superpotential and soft terms. The superpotential (in the UV) is defined as

$$
\begin{aligned}
\left.W\right|_{\mathrm{UV}}= & \left(\mu+\lambda_{S} S\right)\left(h_{u}^{A} h_{d}^{A}+h_{u}^{B} h_{d}^{B}\right)+\lambda_{A} S_{A} h_{u}^{A} h_{d}^{A} \\
& +y_{t}\left(Q_{3}^{A} U_{3}^{A} h_{u}^{A}+Q_{3}^{B} U_{3}^{B} h_{u}^{B}\right)+\frac{1}{2} M_{S} S^{2}+\frac{1}{2} M_{S_{A}} S_{A}^{2} \\
& +\lambda_{B D} B h_{u}^{A} h_{u}^{B}+M_{B} \bar{B} B
\end{aligned}
$$

where $B$ is a bi-doublet under $\mathrm{SU}(2)_{A} \times \mathrm{SU}(2)_{B}$ and is charged under $\mathrm{U}(1)_{A} \times \mathrm{U}(1)_{B}$ as $(-1 / 2,-1 / 2)$, while $\bar{B}$ has conjugate quantum numbers. Here we have set to zero the mixed $S$ - $S_{A}$ mass term, a $\mathbb{Z}_{2}$-breaking $\mu$-term and $\mathbb{Z}_{2}$-breaking top Yukawa and $S$ couplings, as well as the $S_{A}$ couplings to the $B$-sector . All these operators are generated in the IR as a result of the $\mathbb{Z}_{2}$-breaking couplings $\lambda_{A}$, but remain small as far as $\lambda_{A}$ is not too large:

$$
\begin{aligned}
\left.\delta W\right|_{\mathrm{IR}}= & M_{S S_{A}} S S_{A}+\lambda_{B} S_{A} h_{u}^{B} h_{d}^{B} \\
& +\left(\Delta \mu+\Delta \lambda_{S} S\right)\left(h_{u}^{A} h_{d}^{A}-h_{u}^{B} h_{d}^{B}\right)+\Delta y_{t}\left(Q_{3}^{A} U_{3}^{A} h_{u}^{A}-Q_{3}^{B} U_{3}^{B} h_{u}^{B}\right) .
\end{aligned}
$$

Soft breaking masses (in the UV) are defined as

$$
\begin{aligned}
\left.V_{\mathrm{soft}}\right|_{\mathrm{UV}}= & m_{H_{u}}^{2}\left(\left|h_{u}^{A}\right|^{2}+\left|h_{u}^{B}\right|^{2}\right)+m_{H_{d}}^{2}\left(\left|h_{d}^{A}\right|^{2}+\left|h_{d}^{B}\right|^{2}\right) \\
& +m_{Q}^{2}\left(\left|Q_{3}^{A}\right|^{2}+\left|Q_{3}^{B}\right|^{2}\right)+m_{U}^{2}\left(\left|U_{3}^{A}\right|^{2}+\left|U_{3}^{B}\right|^{2}\right) \\
& -b\left(h_{u}^{A} h_{d}^{A}+h_{u}^{B} h_{d}^{B}+\text { h.c. }\right)+m_{S}^{2}|S|^{2}+m_{S_{A}}^{2}\left|S_{A}\right|^{2} \\
& +m_{B}^{2}|B|^{2}+m_{\bar{B}}^{2}|\bar{B}|^{2}
\end{aligned}
$$


where we have set to zero the mixed $S$ - $S_{A}$ soft mass, the $\mathbb{Z}_{2}$-breaking stop masses and soft Higgs masses and the $b$-term. All these operators are generated in the IR as a result of the $\mathbb{Z}_{2}$-breaking couplings $\lambda_{A}$, but remain small as long as $\lambda_{A}$ is not too large:

$$
\begin{aligned}
\left.\delta V_{\text {soft }}\right|_{\mathrm{IR}}= & m_{S S_{A}}^{2}\left(S S_{A}^{*}+\text { h.c. }\right)-\Delta b\left(h_{u}^{A} h_{d}^{A}-h_{u}^{B} h_{d}^{B}+\text { h.c. }\right) \\
& +\Delta m_{H_{u}}^{2}\left(\left|h_{u}^{A}\right|^{2}-\left|h_{u}^{B}\right|^{2}\right)+\Delta m_{H_{d}}^{2}\left(\left|h_{d}^{A}\right|^{2}-\left|h_{d}^{B}\right|^{2}\right) \\
& +\Delta m_{Q}^{2}\left(\left|Q_{3}^{A}\right|^{2}-\left|Q_{3}^{B}\right|^{2}\right)+\Delta m_{U}^{2}\left(\left|U_{3}^{A}\right|^{2}-\left|U_{3}^{B}\right|^{2}\right) .
\end{aligned}
$$

In the RGEs we can then neglect the contributions of $\mathbb{Z}_{2}$-breaking couplings except $\lambda_{A}$ (that is the only $\mathbb{Z}_{2}$-breaking coupling already present in the UV), as well as the contributions from (SUSY and soft) mixed singlet masses.

Beta functions. The beta function coefficients for the gauge couplings $\tilde{g}_{Y}=g_{Y}, \tilde{g}_{2}=$ $g_{2}, \tilde{g}_{3}=g_{3}$ are:

$$
b_{i}^{\text {hard }}=(11,1,-3), \quad b_{i}^{\text {hard }+ \text { bidoublets }}=(13,3,-3) .
$$

\section{Yukawa couplings}

$$
\begin{aligned}
16 \pi^{2} \frac{d}{d t} \lambda_{S} & \approx \lambda_{S}\left[6 \lambda_{S}^{2}+3 y_{t}^{2}+2 \lambda_{A}^{2}-g_{Y}^{2}-3 g_{2}^{2}+2 \lambda_{B D}^{2}\right], \\
16 \pi^{2} \frac{d}{d t} \Delta \lambda_{S} & \approx 2 \lambda_{S} \lambda_{A}^{2} \\
16 \pi^{2} \frac{d}{d t} \lambda_{A} & \approx \lambda_{A}\left[4 \lambda_{A}^{2}+4 \lambda_{S}^{2}+3 y_{t}^{2}-g_{Y}^{2}-3 g_{2}^{2}+2 \lambda_{B D}^{2}\right], \\
16 \pi^{2} \frac{d}{d t} \lambda_{B} & \approx 2 \lambda_{A} \lambda_{S}^{2} \\
16 \pi^{2} \frac{d}{d t} y_{t} & \approx y_{t}\left[6 y_{t}^{2}+\lambda_{S}^{2}+\frac{1}{2} \lambda_{A}^{2}-\frac{13}{9} g_{Y}^{2}-3 g_{2}^{2}-\frac{16}{3} g_{3}^{2}+2 \lambda_{B D}^{2}\right], \\
16 \pi^{2} \frac{d}{d t} \Delta y_{t} & \approx \frac{1}{2} y_{t} \lambda_{A}^{2} \\
16 \pi^{2} \frac{d}{d t} \lambda_{B D} & =\lambda_{B D}\left[5 \lambda_{B D}^{2}+2 \lambda_{S}^{2}+\lambda_{A}^{2}-2 g_{Y}^{2}-6 g_{2}^{2}+6 y_{t}^{2}\right] .
\end{aligned}
$$

\section{SUSY masses}

$$
\begin{aligned}
16 \pi^{2} \frac{d}{d t} \mu & \approx \mu\left[3 y_{t}^{2}+\lambda_{A}^{2}+2 \lambda_{S}^{2}-g_{Y}^{2}-3 g_{2}^{2}+2 \lambda_{B D}^{2}\right] \\
16 \pi^{2} \frac{d}{d t} \Delta \mu & \approx \lambda_{A}^{2} \mu \\
16 \pi^{2} \frac{d}{d t} M_{S} & \approx 8 \lambda_{S}^{2} M_{S} \\
16 \pi^{2} \frac{d}{d t} M_{S_{A}} & \approx 4 \lambda_{A}^{2} M_{S_{A}} \\
16 \pi^{2} \frac{d}{d t} M_{S S_{A}} & \approx 2 \lambda_{A} \lambda_{S}\left(M_{S}+M_{S_{A}}\right) \\
16 \pi^{2} \frac{d}{d t} M_{B} & =M_{B}\left[\left(\lambda_{B D}\right)^{2}-6 g_{2}^{2}-2 g_{Y}^{2}\right] .
\end{aligned}
$$




\section{Soft Masses}

$$
\begin{aligned}
16 \pi^{2} \frac{d}{d t} m_{H_{u}}^{2} & \approx g_{Y}^{2} \xi+2 \lambda_{S}^{2} X_{S}+\lambda_{A}^{2} X_{S_{A}}+6 y_{t}^{2} X_{t}+4 \lambda_{B D}^{2}\left[m_{B}^{2}+2 m_{H_{u}}^{2}\right] \\
16 \pi^{2} \frac{d}{d t} \Delta m_{H_{u}}^{2} & \approx \lambda_{A}^{2} X_{S_{A}}, \\
16 \pi^{2} \frac{d}{d t} m_{H_{d}}^{2} & \approx-g_{Y}^{2} \xi+2 \lambda_{S}^{2} X_{S}+\lambda_{A}^{2} X_{S_{A}} \\
16 \pi^{2} \frac{d}{d t} \Delta m_{H_{d}}^{2} & \approx \lambda_{A}^{2} X_{S_{A}} \\
16 \pi^{2} \frac{d}{d t} m_{Q_{3}}^{2} & =\frac{1}{3} g_{Y}^{2} \xi-\frac{32}{3} g_{3}^{2} M_{3}^{2}+2 y_{t}^{2} X_{t} \\
16 \pi^{2} \frac{d}{d t} \Delta m_{Q_{3}}^{2} & \approx 0 \\
16 \pi^{2} \frac{d}{d t} m_{U_{3}}^{2} & =-\frac{4}{3} g_{Y}^{2} \xi-\frac{32}{3} g_{3}^{2} M_{3}^{2}+4 y_{t}^{2} X_{t} \\
16 \pi^{2} \frac{d}{d t} \Delta m_{U_{3}}^{2} & \approx 0 \\
16 \pi^{2} \frac{d}{d t} m_{S}^{2} & \approx 8 \lambda_{S}^{2} X_{S}, \\
16 \pi^{2} \frac{d}{d t} m_{S_{A}}^{2} & \approx 4 \lambda_{A}^{2} X_{S_{A}}, \\
16 \pi^{2} \frac{d}{d t} m_{S S_{A}}^{2} & \approx 2 \lambda_{S} \lambda_{A}\left(X_{S}+X_{S_{A}}\right) \\
16 \pi^{2} \frac{d}{d t} m_{B}^{2} & =-2 g_{Y}^{2} \xi+2 \lambda_{B D}^{2}\left[m_{B}^{2}+2 m_{H_{u}}^{2}\right] \\
16 \pi^{2} \frac{d}{d t} m_{\bar{B}}^{2} & =2 g_{Y}^{2} \xi
\end{aligned}
$$

with the auxiliary functions

$$
\begin{aligned}
X_{S} & \equiv m_{S}^{2}+m_{H_{u}}^{2}+m_{H_{d}}^{2}, \\
X_{S_{A}} & \equiv m_{S_{A}}^{2}+m_{H_{u}}^{2}+m_{H_{d}}^{2}, \\
X_{t} & \equiv m_{Q}^{2}+m_{U}^{2}+m_{H_{u}}^{2}, \\
\xi & \equiv 3 m_{Q}^{2}-6 m_{U}^{2}+m_{H_{u}}^{2}-m_{H_{d}}^{2}+2 m_{\bar{B}}^{2}-2 m_{B}^{2} .
\end{aligned}
$$

Moreover one has

$$
\begin{aligned}
16 \pi^{2} \frac{d}{d t} b & \approx b\left[10 \lambda_{S}^{2}+3 \lambda_{A}^{2}+3 y_{t}^{2}-g_{Y}^{2}-3 g_{2}^{2}+2 \lambda_{B D}^{2}\right], \\
16 \pi^{2} \frac{d}{d t} \Delta b & =3 \lambda_{A}^{2} b \\
16 \pi^{2} \frac{d}{d t} M_{3} & =-6 g_{3}^{2} M_{3} .
\end{aligned}
$$

\section{B Higgs sector spectrum}

In this appendix we give further details about the (SUSY) Twin Higgs model. First we fully solve the Twin Higgs model as a linear sigma model deriving exact formulas for the VEVs, 
the masses and the mixing in order to study the validity regime of the PGB approximation. Then we provide the analytical formulae describing the phenomenology of the Twin SUSY Higgs sector when some hierarchy is present between the twin Higgs and the SUSY Higgses (i.e. for $\lambda_{S} f \ll m_{A}$ ): mass eigenvalues, eigenvectors and decay widths.

\section{B.1 The Twin Higgs as a linear sigma model}

In section 2 we have explored the parameter space of the Twin Higgs in complete generality, integrating out the radial mode and obtaining a non-linear sigma model description of the SM Higgs as a PGB of the spontaneously broken SU(4)-symmetry. Here we repeat the same exercise working directly at the level of the linear sigma model with both the radial mode/twin Higgs and the SM-like Higgs in the spectrum. We obtain fully general formulas for the mass of the twin Higgs and the SM-like Higgs mass and for their mixing. We show how the PGB formulas are obtained expanding these expressions at leading order in $k, \sigma \ll \lambda$.

The Twin potential can be defined in terms of five parameters $\left\{\lambda, \rho, \kappa, m^{2}, \tilde{\mu}^{2}\right\}$

$$
\begin{aligned}
V_{T}= & \lambda\left(\left|H_{A}\right|^{2}+\left|H_{B}\right|^{2}\right)^{2}+m^{2}\left(\left|H_{A}\right|^{2}+\left|H_{B}\right|^{2}\right) \\
& +\kappa\left(\left|H_{A}\right|^{4}+\left|H_{B}\right|^{4}\right)+\tilde{\mu}^{2}\left|H_{A}\right|^{2}+\rho\left|H_{A}\right|^{4} .
\end{aligned}
$$

The minimization conditions set the VEVs of $H_{A}$ and $H_{B}\left(v_{A}\right.$ and $\left.v_{B}\right)$ as a function of these parameters. Defining $v_{A}^{2}=v^{2}$ and $v_{B}^{2}=f^{2}-v^{2}$ in analogy with the PGB approximation, one obtains with the shorthand notation $\sigma \equiv-2 \lambda \tilde{\mu}^{2} / m^{2}$

$$
v^{2}=-\frac{m^{2}}{4} \frac{-\sigma+\kappa(2-\sigma / \lambda)}{\lambda \rho+\kappa(2 \lambda+\rho+\kappa)}, \quad f^{2}=-\frac{m^{2}}{4} \frac{2 \rho+\kappa(4-\sigma / \lambda)}{\lambda \rho+\kappa(2 \lambda+\rho+\kappa)} .
$$

Note that in the limit $\kappa \ll \lambda$ one has $m^{2}=-2 \lambda f^{2}$, thus recovering the definition of $\sigma$ in section 2. Moreover, in the limit $\kappa, \sigma \ll \lambda$ eq. (B.2) reproduces the PGB formula in eq. (2.3). We can use the above equations to trade the two VEVs $v$ and $f$ for two Lagrangian parameters, for instance the two scales $m^{2}$ and $\tilde{\mu}^{2}$, or equivalently $m^{2}$ and $\sigma$.

We are now interested in the mass spectrum, in particular the mass of the lightest (PGB) Higgs $h$. The full analytic expression for the masses of the two physical Higgs bosons is given by

$$
m_{h, H}^{2}=2\left[\rho v^{2}+f^{2}(\lambda+\kappa)(1 \mp \sqrt{1-\mathcal{S}})\right]
$$

with

$$
\mathcal{S}=2 \frac{v^{2}}{f^{2}} \frac{\lambda \rho+\kappa(4 \lambda+\rho+2 \kappa)}{(\lambda+\kappa)^{2}}-\frac{v^{4}}{f^{4}} \frac{4 \lambda \rho+\rho^{2}+\kappa(8 \lambda+4 \rho+4 \kappa)}{(\lambda+\kappa)^{2}} .
$$

Expanding in $v \ll f$ and $\kappa \ll \lambda$ the square root in eq. (B.3) gives

$$
\sqrt{1-\mathcal{S}} \approx 1-\frac{\rho+\kappa(4-\rho / \lambda)}{\lambda} \frac{v^{2}}{f^{2}}+\frac{2 \rho}{\lambda} \frac{v^{4}}{f^{4}}
$$


Another useful approximation is to expand at leading order in $\kappa / \lambda$ and $\rho / \lambda$, but keep all orders in $v / f$. In this way we get for the Higgs mass

$$
m_{h}^{2} \approx 4 v^{2}\left(1-\frac{v^{2}}{f^{2}}\right)\left[(2 \kappa+\rho)-\frac{\left(f^{2} \kappa-v^{2}(2 \kappa+\rho)\right)^{2}}{\lambda f^{4}}\right],
$$

which shows that the PGB expression in eq. (2.4) tends to overestimate the mass of the SM Higgs. In the same approximation the mass of radial mode is

$$
m_{H}^{2} \approx 4 f^{2} \lambda\left[1+\frac{f^{4} \kappa-2 f^{2} \kappa v^{2}+v^{4}(2 \kappa+\rho)}{\lambda f^{4}}\right] .
$$

Defining the mixing angle $\theta$ by $\left(c_{\theta} \equiv \cos \theta, s_{\theta} \equiv \sin \theta\right)$

$$
h=c_{\theta} h_{A}-s_{\theta} h_{B}, \quad H=s_{\theta} h_{A}+c_{\theta} h_{B},
$$

we find in the same approximation

$$
s_{\theta}^{2} \approx \frac{v^{2}}{f^{2}}\left[1-2\left(1-\frac{v^{2}}{f^{2}}\right) \frac{\kappa f^{2}-v^{2}(2 \kappa+\rho)}{\lambda f^{2}}\right] .
$$

\section{B.2 The SUSY Twin Higgs}

The SUSY Twin Higgs is a double copy of the MSSM. The scalar potential can be schematically divided into three parts, depending if they break $\mathrm{U}(4)$ and/or the $\mathbb{Z}_{2}$ symmetry

$$
\begin{aligned}
V= & V_{\mathrm{U}(4)}+V_{\Psi(4), Z_{2}}+V_{\Psi(4), Z_{2}} \\
V_{\mathrm{U}(4)}= & \lambda_{S}^{2}\left|h_{u}^{A} h_{d}^{A}+h_{u}^{B} h_{d}^{B}\right|^{2}+m_{u}^{2}\left(\left|h_{u}^{A}\right|^{2}+\left|h_{u}^{B}\right|^{2}\right)+m_{d}^{2}\left(\left|h_{d}^{A}\right|^{2}+\left|h_{d}^{B}\right|^{2}\right) \\
V_{\Psi(4), Z_{2}}= & \frac{g_{\mathrm{ew}}^{2}}{8}\left[\left(\left|h_{d}^{A}\right|^{2}-\left|h_{u}^{A}\right|^{2}\right)^{2}+\left(\left|h_{d}^{B}\right|^{2}-\left|h_{u}^{B}\right|^{2}\right)^{2}\right]-b\left(h_{u}^{A} h_{d}^{A}+h_{u}^{B} h_{d}^{B}+\text { h.c. }\right) \\
& +\delta \lambda_{u}\left(\left|h_{u}^{A}\right|^{4}+\left|h_{u}^{B}\right|^{4}\right)+\lambda_{B D}^{2}\left|h_{u}^{A}\right|^{2}\left|h_{u}^{B}\right|^{2} \\
V_{\Psi(4), Z_{2}}= & \lambda_{A}^{2}\left|h_{u}^{A} h_{d}^{A}\right|^{2}+\Delta m_{u}^{2}\left|h_{u}^{A}\right|^{2}+\Delta m_{d}^{2}\left|h_{d}^{A}\right|^{2}+\delta \rho_{u}\left|h_{u}^{A}\right|^{4}
\end{aligned}
$$

where we have defined $g_{\text {ew }}^{2}=g^{2}+g^{\prime 2}$. Apart from the soft $\mathbb{Z}_{2}$-breaking terms $\Delta m_{u, d}^{2}$, hard breaking terms $\lambda_{A}^{2}$ and bi-doublet terms $\lambda_{B D}^{2}$, we have included the terms $\delta \lambda_{u}$ and $\delta \rho_{u}$ that are generated at one-loop from the stop/top sector, with

$$
\delta \lambda_{u} \approx \frac{3 m_{t}^{4}}{16 \pi^{2} s_{\beta}^{4} v^{4}} \log \frac{M_{S}^{2}}{m_{t_{B}}^{2}}, \quad \delta \rho_{u} \approx \frac{3 m_{t}^{4}}{16 \pi^{2} s_{\beta}^{4} v^{4}} \log \frac{f^{2}}{v^{2}} .
$$

We then minimize the potential and trade the parameters $\Delta m_{u}^{2}, \Delta m_{d}^{2}, m_{u}^{2}, m_{d}^{2}$ for $v^{2}, f^{2}$ and $t_{\beta} \equiv \tan \beta_{A} \approx \tan \beta_{B}$. We do not take into account corrections proportional to $\delta \tan \beta=\tan \beta_{A}-\tan \beta_{B}$, which typically lead only to small corrections. We can then compute the mass eigenvalues in the $\mathrm{CP}$-even sector, in the CP-odd sector and for the charged Higgses. These latter results are particularly simple, since the CP-odd and charged Higgs mass matrices are $2 \times 2$ matrices that can be diagonalized exactly. 
CP-odd Higgs sector. The CP-odd Higgs eigenvalues and eigenvectors are

$$
\begin{aligned}
m_{A_{T}}^{2} & =\frac{2 b}{s_{2 \beta}}, \\
m_{A}^{2} & =m_{A_{T}}^{2}-\lambda_{S}^{2} f^{2}, \\
A_{T} & =\frac{v}{f} c_{\beta} a_{u}^{A}+\frac{v}{f} s_{\beta} a_{d}^{A}+c_{\beta} \sqrt{1-\frac{v^{2}}{f^{2}}} a_{u}^{B}+s_{\beta} \sqrt{1-\frac{v^{2}}{f^{2}}} a_{d}^{B}, \\
A & =-c_{\beta} \sqrt{1-\frac{v^{2}}{f^{2}}} a_{u}^{A}-s_{\beta} \sqrt{1-\frac{v^{2}}{f^{2}}} a_{d}^{A}+\frac{v}{f} c_{\beta} a_{u}^{B}+\frac{v}{f} s_{\beta} a_{d}^{B},
\end{aligned}
$$

where we defined $A_{T}$ as the CP-odd state that is mainly dark for $v^{2} / f^{2} \ll 1$.

Charged Higgs sector. The charged Higgs eigenvalues and eigenvectors are given by

$$
\begin{aligned}
m_{H_{T}^{ \pm}}^{2} & =m_{A}^{2}, \\
m_{H^{ \pm}}^{2} & =m_{A}^{2}-\lambda_{A}^{2} v^{2}, \\
H_{T}^{-} & =c_{\beta}\left(h_{u}^{+B}\right)^{*}+s_{\beta} h_{d}^{-B}, \\
H^{-} & =c_{\beta}\left(h_{u}^{+A}\right)^{*}+s_{\beta} h_{d}^{-A} .
\end{aligned}
$$

Note that gauge invariance forbids mixing between the visible and dark charged Higgs.

CP-even Higgs sector. The CP-even sector mass matrix is a $4 \times 4$ matrix whose exact diagonalization expressions are not very illuminating. In the main text we compute the eigenvalues and eigenvectors numerically to perform the phenomenological study. In this appendix we provide analytic expressions in some simplifying limits.

As we already discussed in section 4, the SUSY Twin Higgs model possesses two different regimes of the Higgs mass parameters which give a different hierarchy in the mass spectrum. For $m_{A}^{2} \gg \lambda_{S}^{2} f^{2}$ the next to lightest state after the SM Higgs boson is the twin Higgs, which belongs to the dark sector and develops a VEV of order $f$. In the regime where $m_{A}^{2} \gtrsim \lambda_{S}^{2} f^{2}$ the lightest scalar above the SM-like higgs is instead the MSSM-like heavy scalar. In this limit the model resembles the MSSM with a CP-odd mass scale set by the combination $m_{A}^{2}=m_{A_{T}}^{2}-\lambda^{2} f^{2}$.

In order to obtain expressions for the masses that can describe the transition between these two different regimes, we consider the simplifying limit $v \rightarrow 0$ and keep only leading order terms in $g_{\mathrm{ew}}^{2}$ and $\delta \lambda_{u}$. In this approximation the mass eigenvalues are

$$
\begin{aligned}
m_{h}^{2} & =0 \\
m_{H}^{2} & =m_{A}^{2}=m_{A_{T}}^{2}-\lambda_{S}^{2} f^{2}, \\
m_{h_{T}}^{2} & =\frac{1}{4}\left(2 m_{A_{T}}^{2}+g_{\mathrm{ew}}^{2} f^{2}+8 \delta \lambda_{u} f^{2} s_{\beta}^{2}-2 m_{A_{T}}^{2} \mathcal{R}\right), \\
m_{H_{T}}^{2} & =\frac{1}{4}\left(2 m_{A_{T}}^{2}+g_{\mathrm{ew}}^{2} f^{2}+8 \delta \lambda_{u} f^{2} s_{\beta}^{2}+2 m_{A_{T}}^{2} \mathcal{R}\right), \\
\mathcal{R} & \equiv \sqrt{1-\frac{f^{2}}{m_{A_{T}}^{2}}\left(4 \lambda_{S}^{2} s_{2 \beta}^{2}+g_{\mathrm{ew}}^{2} c_{4 \beta}-8 \delta \lambda_{u} c_{2 \beta} s_{\beta}^{2}\right)+\frac{2 \lambda_{S}^{2} f^{4}}{m_{A_{T}}^{4}}\left(2 \lambda_{S}^{2}-g_{\mathrm{ew}}^{2}\right) s_{2 \beta}^{2}} .
\end{aligned}
$$


We can expand these expressions in the two different regimes. For $\lambda_{S}^{2} f^{2} \ll m_{A_{T}}^{2}$ we get

$$
\begin{aligned}
& m_{h_{T}}^{2}=f^{2}\left(\lambda_{S}^{2} s_{2 \beta}^{2}+\frac{1}{2} g_{\mathrm{ew}}^{2} c_{2 \beta}^{2}+4 \delta \lambda_{u} s_{\beta}^{4}\right) \\
& m_{H_{T}}^{2}=m_{A_{T}}^{2}-f^{2} s_{2 \beta}^{2}\left(\lambda_{S}^{2}-\frac{1}{2} g_{\mathrm{ew}}^{2}-\delta \lambda_{u}\right) .
\end{aligned}
$$

Here the twin Higgs is lighter than the MSSM-like state. On the other hand, for small $m_{A_{T}}^{2}$ but still with $m_{A_{T}}^{2} \gtrsim \lambda_{S}^{2} f^{2}$ we get

$$
\begin{aligned}
& m_{h_{T}}^{2}=\frac{1}{2} f^{2} g_{\mathrm{ew}}^{2}+s_{2 \beta}^{2}\left(m_{A_{T}}^{2}-\lambda_{S}^{2} f^{2}\right)+4 \delta \lambda_{u} f^{2} s_{\beta}^{4}, \\
& m_{H_{T}}^{2}=\lambda_{S}^{2} f^{2}+f^{2}\left[\lambda_{S}^{2} c_{2 \beta}^{2}\left(1-\frac{\lambda_{S}^{2} f^{2}}{m_{A_{T}}^{2}}\right)+\delta \lambda_{u} s_{2 \beta}^{2}\right] .
\end{aligned}
$$

Contrary to the previous regime, here the Twin Higgs is always heavier than the MSSM-like Higgs, and their mass splitting is set by

$$
m_{h_{T}}^{2}-m_{H}^{2}=f^{2}\left(\frac{1}{2} g_{\mathrm{ew}}^{2}-\lambda_{S}^{2} c_{2 \beta}^{2}\left(1-\frac{\lambda_{S}^{2} f^{2}}{m_{A_{T}}^{2}}\right)+4 \delta \lambda_{u} s_{\beta}^{4}\right) .
$$

To make the transition manifest, we plot in figure 14 the eigenvalues as a function of $f / v$ fixing the other parameters. ${ }^{17}$ The transition between the two regimes happens approximately at $m_{A}^{2} \approx \lambda_{S}^{2} f^{2}$. For $\lambda_{S}^{2} f^{2}$ smaller than this critical value the lightest eigenstate is the twin Higgs, while for larger $\lambda_{S}^{2} f^{2}$ it is MSSM-like. For completeness we compare the analytic expressions with the numerical results where $v$ is turned on. On the same plot in figure 14 we show in dashed the numerical eigenvalues as a function of $f / v$ on the same benchmark, showing that $v \neq 0$ only results in a slight separation of the eigenvalues in the transition regime.

In order to better understand the phenomenology in section 4 we plot in figure 15 the mixing angles of the two mass eigenstates $\left\{H, h_{T}\right\}$ with respect to the gauge eigenbasis $\left\{h_{u}^{A}, h_{d}^{A}, h_{u}^{B}, h_{d}^{B}\right\}$. In the left panel of figure 15 we focus on the $B$-components into the dark sector and denote with solid blue/red lines the $u / d$-components the next-to-lightest eigenvalues. The dashed lines refer instead to the third lightest eigenvalue with the same color coding (blue for $u$ - and red for $d$-components). In the right panel of figure 15 we plot with the same color coding the $A$-components into the visible sector (blue for $u$ - and red for $d$ - components).

From these plots it is clear that the role of the eigenvalues is inverted in the transition region around $\lambda_{S} f \approx m_{A}$. For $\lambda_{S} f \lesssim m_{A}$ the next-to-lightest eigenstate is the twin-Higgs, while for $\lambda_{S} f \gtrsim m_{A}$ it is MSSM-like. In the transition region with $\lambda_{S} f \approx m_{A}$ all states are sizeably mixed one each other.

The visible sector content of the extra Higgs states determines their production cross section at the LHC and their visible branching ratio.

\footnotetext{
${ }^{17}$ As explained in the text, $v$ is set to zero for this plot so the $\mathrm{x}$-axis should be understood as $f$ in units of $174 \mathrm{GeV}$.
} 


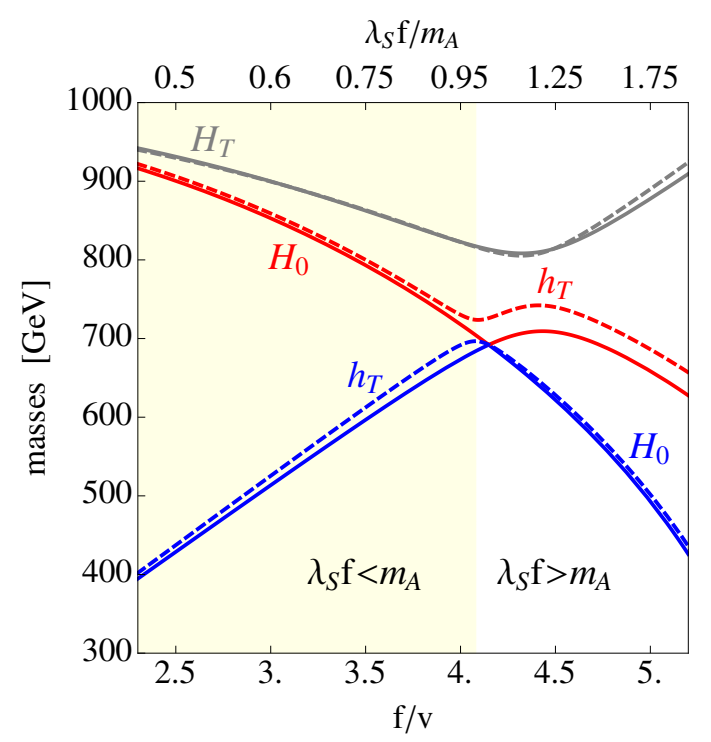

Figure 14. Left: mass eigenvalues as a function of $f / v$. We fix $\tan \beta=1.2, m_{A_{T}}=1 \mathrm{TeV}$ and $\lambda_{S}=1$. The solid lines are obtained using the analytic expressions in eq. (B.14) in the $v \rightarrow 0$ limit. The dashed lines are obtained numerically including $v / f$ corrections. The light yellow/white area correspond to the region where $\lambda_{S} f \lessgtr m_{A}$ as indicated in the plot.
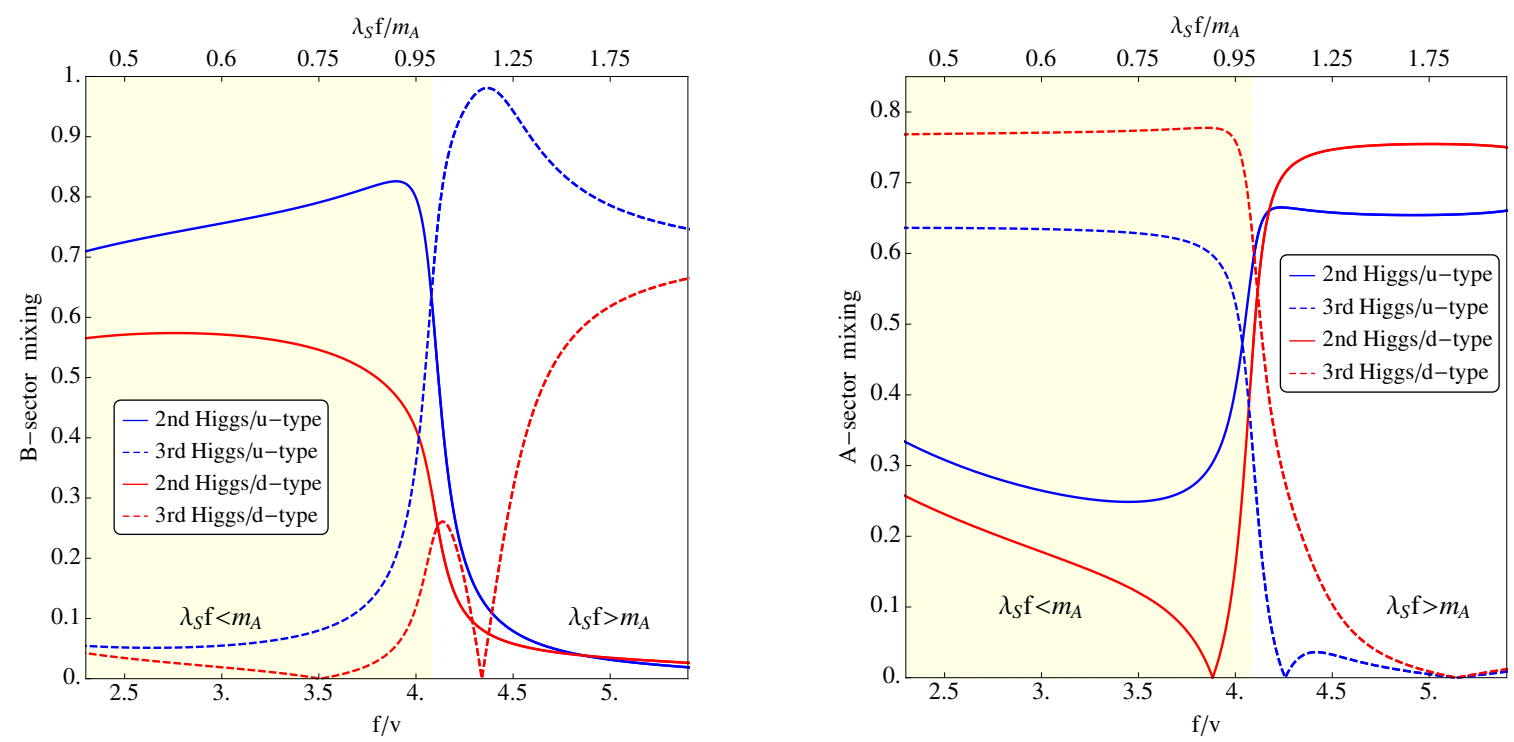

Figure 15. Mixing as a function of $f / v$. We fix $\tan \beta=1.2, m_{A_{T}}=1 \mathrm{TeV}, \lambda_{S}=1$. The light yellow/white area correspond to the region where $\lambda_{S} f \lessgtr m_{A}$ as indicated in the plot. Left: $B$ sector components of the next to lightest (thick) and next to next to lightest (dashed) CP-even Higgs. The blue is the $h_{u}^{B}$ component while the red is the $h_{d}^{B}$ one. Right: $A$-sector components of the next to lightest (thick) and next to next to lightest (dashed) eigenstate. The blue is the $h_{u}^{A}$ component while the red is the $h_{d}^{A}$ one. 
For small $\lambda_{S} f \lesssim m_{A}$ the twin Higgs is the next-to-lightest Higgs and it gets sizeable couplings to the visible sector through its component along $h_{u}^{A}$. As a consequence it can be produced at the LHC in gluon fusion and it gives rise to interesting signals in di-boson channels. In the transition region, when $\lambda_{S} f \approx m_{A}$, there is an enhancement of the $h_{u}^{A}$ components as it can be seen from the solid blue line in the right panel of figure 15 . We then expect a slight improvement in the sensitivity on the twin Higgs searches at LHC into di-bosons. For $\lambda_{S} f \gtrsim m_{A}$ the next-to-lightest state becomes MSSM-like and the sensitivity of the di-boson searches drops since MSSM-like have very small branching ratio into gauge bosons and at the same time the twin Higgs has a small mixing angle with the visible sector as it can be seen from the dashed lines in the right panel figure 15. This argument explains the horn-like shape of the di-boson exclusion regions in figure 12.

\section{B.3 Approximate expressions for eigenvalues and eigenvectors}

We have seen that in the regime $\lambda_{S}^{2} f^{2} \ll m_{A}^{2}$ the collider phenomenology of the model is characterised by a light twin Higgs with interesting signatures. In this regime we can expand the expressions for masses and mixings for large $m_{A}$ (or equivalently large $m_{A_{T}}$ ) and obtain approximate analytical results. From the scalar potential (B.10) we get for the spectrum, keeping only the leading order terms in $g_{\mathrm{ew}}^{2}, \lambda_{A}^{2}, \lambda_{B D}^{2}, \delta \lambda_{u}, \delta \rho_{u}$ and $f^{2} / m_{A_{T}}^{2}$ :

$$
\begin{aligned}
m_{h}^{2} & =v^{2}\left(1-\frac{v^{2}}{f^{2}}\right)\left(g_{\mathrm{ew}}^{2} c_{2 \beta}^{2}+\lambda_{A}^{2} s_{2 \beta}^{2}-4 \lambda_{B D}^{2} s_{\beta}^{4}+8 \delta \lambda_{u} s_{\beta}^{4}+4 \delta \rho_{u} s_{\beta}^{4}\right) \\
m_{h_{T}}^{2} & =\lambda_{S}^{2} f^{2} s_{2 \beta}^{2}+\frac{f^{2}}{2}\left(g_{\mathrm{ew}}^{2} c_{2 \beta}^{2}+8 \delta \lambda_{u} s_{\beta}^{4}\right)+4 \lambda_{B D}^{2} v^{2} s_{\beta}^{4}+\frac{v^{4}}{f^{2}}\left(\lambda_{A}^{2} s_{2 \beta}^{2}+4 \delta \rho_{u} s_{\beta}^{4}\right) \\
m_{H_{T}}^{2} & =m_{A_{T}}^{2}-f^{2} s_{2 \beta}^{2}\left(\lambda_{S}^{2}-\frac{1}{2} g_{\mathrm{ew}}^{2}-\delta \lambda_{u}\right)+\lambda_{B D}^{2} v^{2} s_{2 \beta}^{2}-\frac{v^{4}}{f^{2}} s_{2 \beta}^{2}\left(\lambda_{A}^{2}-\delta \rho_{u}\right) \\
m_{H}^{2} & =m_{A_{T}}^{2}-\lambda_{S}^{2} f^{2}+v^{2} s_{2 \beta}^{2}\left(g_{\mathrm{ew}}^{2}-\lambda_{A}^{2}-\lambda_{B D}^{2}+2 \delta \lambda_{u}+\delta \rho_{u}\right)
\end{aligned}
$$

Similarly we can calculate the mixing angles in the same approximation. Defining the rotation matrix $V$ as

$$
\left(\begin{array}{c}
h \\
h_{T} \\
H_{T} \\
H
\end{array}\right)=V\left(\begin{array}{c}
h_{u}^{A} \\
h_{d}^{A} \\
h_{u}^{B} \\
h_{d}^{B}
\end{array}\right)
$$

the entries are, keeping only the leading order terms in $g_{\mathrm{ew}}^{2}, \lambda_{A}^{2}, \lambda_{B D}^{2}, \delta \lambda_{u}, \delta \rho_{u}$ and $f^{2} / m_{A_{T}}^{2}$ :

$$
\begin{aligned}
h= & {\left[s_{\beta}\left(1-\frac{v^{2}}{2 f^{2}}+\frac{\lambda_{1}^{2} v^{2}}{2 \lambda_{S}^{2} f^{2} t_{2 \beta}^{2}}\right)\right] h_{u}^{A}+\left[c_{\beta}\left(1-\frac{v^{2}}{2 f^{2}}+\frac{\lambda_{1}^{2} v^{2}}{2 \lambda_{S}^{2} f^{2} t_{2 \beta}^{2}}\right)\right] h_{d}^{A} } \\
& +\left[-\frac{v}{f} s_{\beta}\left(1-\frac{\lambda_{1}^{2}}{2 \lambda_{S}^{2} t_{2 \beta}^{2}}\right)\right] h_{u}^{B}+\left[-\frac{v}{f} c_{\beta}\left(1-\frac{\lambda_{1}^{2}}{2 \lambda_{S}^{2} t_{2 \beta}^{2}}\right)\right] h_{d}^{B},
\end{aligned}
$$




$$
\begin{aligned}
h_{T}= & {\left[\frac{v}{f} s_{\beta}\left(1-\frac{\lambda_{1}^{2}}{2 \lambda_{S}^{2} t_{2 \beta}^{2}}\right)\right] h_{u}^{A}+\left[\frac{v}{f} c_{\beta}\left(1-\frac{\lambda_{1}^{2}}{2 \lambda_{S}^{2} t_{2 \beta}^{2}}\right)\right] h_{d}^{A} } \\
& +\left[s_{\beta}\left(1-\frac{v^{2}}{2 f^{2}}+\frac{\lambda_{1}^{2} v^{2}}{2 \lambda_{S}^{2} f^{2} t_{2 \beta}^{2}}\right)\right] h_{u}^{B}+\left[c_{\beta}\left(1-\frac{v^{2}}{2 f^{2}}+\frac{\lambda_{1}^{2} v^{2}}{2 \lambda_{S}^{2} f^{2} t_{2 \beta}^{2}}\right)\right] h_{d}^{B}, \\
H_{T}= & {\left[\frac{v}{f} c_{\beta}\left(1-\frac{\lambda_{2}^{2} t_{2 \beta}^{2}}{2 \lambda_{S}^{2}}\right)\right] h_{u}^{A}+\left[-\frac{v}{f} s_{\beta}\left(1-\frac{\lambda_{2}^{2} t_{2 \beta}^{2}}{2 \lambda_{S}^{2}}\right)\right] h_{d}^{A} } \\
& +\left[c_{\beta}\left(1-\frac{v^{2}}{2 f^{2}}+\frac{\lambda_{2}^{2} v^{2} t_{2 \beta}^{2}}{2 \lambda_{S}^{2} f^{2}}\right)\right] h_{u}^{B}+\left[-s_{\beta}\left(1-\frac{v^{2}}{2 f^{2}}+\frac{\lambda_{2}^{2} v^{2} t_{2 \beta}^{2}}{2 \lambda_{S}^{2} f^{2}}\right)\right] h_{d}^{B}, \\
H= & {\left[c_{\beta}\left(1-\frac{v^{2}}{2 f^{2}}+\frac{\lambda_{2}^{2} v^{2} t_{2 \beta}^{2}}{2 \lambda_{S}^{2} f^{2}}\right)\right] h_{u}^{A}+\left[-s_{\beta}\left(1-\frac{v^{2}}{2 f^{2}}+\frac{\lambda_{2}^{2} v^{2} t_{2 \beta}^{2}}{2 \lambda_{S}^{2} f^{2}}\right)\right] h_{d}^{A} } \\
& +\left[-\frac{v}{f} c_{\beta}\left(1-\frac{\lambda_{2}^{2} t_{2 \beta}^{2}}{2 \lambda_{S}^{2}}\right)\right] h_{u}^{B}+\left[\frac{v}{f} s_{\beta}\left(1-\frac{\lambda_{2}^{2} t_{2 \beta}^{2}}{2 \lambda_{S}^{2}}\right)\right] h_{d}^{B},
\end{aligned}
$$

with the shorthand notation

$$
\begin{aligned}
& \lambda_{1}^{2}=g_{\mathrm{ew}}^{2}-2 \lambda_{A}^{2} \frac{v^{2}}{f^{2}} t_{2 \beta}^{2}-t_{\beta}^{2} t_{2 \beta}^{2}\left(\lambda_{B D}^{2}-2 \delta \lambda_{u}+2 \delta \rho_{u} \frac{v^{2}}{f^{2}}\right), \\
& \lambda_{2}^{2}=g_{\mathrm{ew}}^{2}-\lambda_{B D}^{2}+2 \delta \lambda_{u}+2 \lambda_{A}^{2} \frac{v^{2}}{f^{2}}-2 \delta \rho_{u} \frac{v^{2}}{f^{2}} .
\end{aligned}
$$

\section{B.4 Decay rates}

In the phenomenological study in the main text we make use of several branching ratio for the Higgs sector. Here we report the most relevant formulas used in the analysis.

Decays into fermions. The relevant Lagrangian is given by

$$
\mathcal{L}=\frac{m_{t}}{\sqrt{2} v s_{\beta}}\left(\bar{t}_{A} t_{A} V_{i 1}+\bar{t}_{B} t_{B} V_{i 3}\right) H_{i}+\frac{m_{b}}{\sqrt{2} v c_{\beta}}\left(\bar{b}_{A} b_{A} V_{i 2}+\bar{b}_{B} b_{B} V_{i 4}\right) H_{i},
$$

with Higgs mass eigenstates $H_{i}=h, h_{T}, H_{T}, H$ and the rotation matrix $V$ has been given in the previous section. If kinematically allowed, the decay rates into tops, dark tops, bottoms and dark bottoms are given by:

$$
\begin{aligned}
\Gamma\left(H_{i} \rightarrow t_{A} t_{A}\right) & =\frac{3 m_{t}^{2} M_{H_{i}}}{16 \pi v^{2} s_{\beta}^{2}}\left|V_{i 1}\right|^{2}\left(1-4 \frac{m_{t}^{2}}{M_{H_{i}}^{2}}\right)^{3 / 2}, \\
\Gamma\left(H_{i} \rightarrow t_{B} t_{B}\right) & =\frac{3 m_{t}^{2} M_{H_{i}}}{16 \pi v^{2} s_{\beta}^{2}}\left|V_{i 3}\right|^{2}\left(1-4 \frac{m_{t_{B}}^{2}}{M_{H_{i}}^{2}}\right)^{3 / 2}, \\
\Gamma\left(H_{i} \rightarrow b_{A} b_{A}\right) & =\frac{3 m_{b}^{2} M_{H_{i}}}{16 \pi v^{2} c_{\beta}^{2}}\left|V_{i 2}\right|^{2}\left(1-4 \frac{m_{b}^{2}}{M_{H_{i}}^{2}}\right)^{3 / 2}, \\
\Gamma\left(H_{i} \rightarrow b_{B} b_{B}\right) & =\frac{3 m_{b}^{2} M_{H_{i}}}{16 \pi v^{2} c_{\beta}^{2}}\left|V_{i 4}\right|^{2}\left(1-4 \frac{m_{b_{B}}^{2}}{M_{H_{i}}^{2}}\right)^{3 / 2}
\end{aligned}
$$


Decays into vector bosons. The relevant Lagrangian is given by

$$
\begin{aligned}
\mathcal{L}= & H_{i}\left(\frac{g}{2 c_{W}} M_{Z} Z^{\mu} Z_{\mu}+g M_{W} W^{+\mu} W_{\mu}^{-}\right)\left(s_{\beta} V_{i 1}+c_{\beta} V_{i 2}\right) \\
& +H_{i}\left(\frac{g}{2 c_{W}} M_{Z_{B}} Z_{B}^{\mu} Z_{B \mu}+g M_{W_{B}} W_{B}^{+\mu} W_{B \mu^{-}}\right)\left(s_{\beta} V_{i 3}+c_{\beta} V_{i 4}\right) .
\end{aligned}
$$

If kinematically allowed, the decay rates into visible and dark gauge bosons are given by

$$
\begin{gathered}
\Gamma\left(H_{i} \rightarrow Z Z\right)=\frac{g^{2} M_{H_{i}}^{3}}{128 \pi c_{W}^{2} M_{Z}^{2}}\left|s_{\beta} V_{i 1}+c_{\beta} V_{i 2}\right|^{2}\left(1-4 \frac{M_{Z}^{2}}{M_{H_{i}}^{2}}\right)^{1 / 2}\left(1-4 \frac{M_{Z}^{2}}{M_{H_{i}}^{2}}+12 \frac{M_{Z}^{4}}{M_{H_{i}}^{4}}\right), \\
\Gamma\left(H_{i} \rightarrow W W\right)=\frac{g^{2} M_{H_{i}}^{3}}{64 \pi M_{W}^{2}}\left|s_{\beta} V_{i 1}+c_{\beta} V_{i 2}\right|^{2}\left(1-4 \frac{M_{W}^{2}}{M_{H_{i}}^{2}}\right)^{1 / 2}\left(1-4 \frac{M_{W}^{2}}{M_{H_{i}}^{2}}+12 \frac{M_{W}^{4}}{M_{H_{i}}^{4}}\right), \\
\Gamma\left(H_{i} \rightarrow Z_{B} Z_{B}\right)=\frac{g^{2} M_{H_{i}}^{3}}{128 \pi c_{W}^{2} M_{Z_{B}}^{2}}\left|s_{\beta} V_{i 3}+c_{\beta} V_{i 4}\right|^{2}\left(1-4 \frac{M_{Z_{B}}^{2}}{M_{H_{i}}^{2}}\right)^{1 / 2}\left(1-4 \frac{M_{Z_{B}}^{2}}{M_{H_{i}}^{2}}+12 \frac{M_{Z_{B}}^{4}}{M_{H_{i}}^{4}}\right), \\
\Gamma\left(H_{i} \rightarrow W_{B} W_{B}\right)=\frac{g^{2} M_{H_{i}}^{3}}{64 \pi M_{W_{B}}^{2}}\left|s_{\beta} V_{i 3}+c_{\beta} V_{i 4}\right|^{2}\left(1-4 \frac{M_{W_{B}}^{2}}{M_{H_{i}}^{2}}\right)^{1 / 2}\left(1-4 \frac{M_{W_{B}}^{2}}{M_{H_{i}}^{2}}+12 \frac{M_{W_{B}}^{4}}{M_{H_{i}}^{4}}\right),
\end{gathered}
$$

Decays into Higgs bosons. Restricting to the U(4)-preserving quartic coupling, the relevant Lagrangian is given by

$$
\begin{aligned}
\mathcal{L} \equiv & \frac{\lambda_{S}^{2}}{\sqrt{2}}\left[v s_{\beta} h_{u}^{A} h_{d}^{A} h_{d}^{A}+v c_{\beta} h_{d}^{A} h_{u}^{A} h_{u}^{A}+\sqrt{f^{2}-v^{2}} s_{\beta} h_{u}^{B} h_{d}^{B} h_{d}^{B}+\sqrt{f^{2}-v^{2}} c_{\beta} h_{d}^{B} h_{u}^{B} h_{u}^{B}\right] \\
& +\frac{\lambda_{S}^{2}}{\sqrt{2}}\left[v s_{\beta} h_{d}^{A} h_{u}^{B} h_{d}^{B}+v c_{\beta} h_{u}^{A} h_{u}^{B} h_{d}^{B}+\sqrt{f^{2}-v^{2}} s_{\beta} h_{u}^{A} h_{d}^{A} h_{d}^{B}+\sqrt{f^{2}-v^{2}} c_{\beta} h_{u}^{A} h_{d}^{A} h_{u}^{B}\right] \\
\equiv & \frac{\lambda_{S}^{2}}{\sqrt{2}} A_{i j k} H_{i} H_{j} H_{k},
\end{aligned}
$$

with

$$
\begin{aligned}
A_{i j k}= & v s_{\beta} V_{i 1} V_{j 2} V_{k 2}+v c_{\beta} V_{k 2} V_{i 1} V_{j 1}+\sqrt{f^{2}-v^{2}} s_{\beta} V_{i 3} V_{j 4} V_{k 4}+\sqrt{f^{2}-v^{2}} c_{\beta} V_{i 4} V_{j 3} V_{k 3} \\
& +v s_{\beta} V_{i 2} V_{j 3} V_{k 4}+v c_{\beta} V_{i 1} V_{j 3} V_{k 4}+\sqrt{f^{2}-v^{2}} s_{\beta} V_{i 1} V_{j 2} V_{k 4}+\sqrt{f^{2}-v^{2}} c_{\beta} V_{i 1} V_{j 2} V_{k 3} .
\end{aligned}
$$

To calculate a given coupling all permutations have to be summed over, e.g. the Lagrangian coupling $\mathcal{L}=A_{h_{T} h h} h_{T} h h$ is given by $A_{h_{T} h h}=\frac{\lambda_{S}^{2}}{\sqrt{2}}\left(A_{122}+A_{212}+A_{221}\right)$. From the general expression

$$
\mathcal{L}=A_{h} H h h+A_{h_{1} h_{2}} H h_{1} h_{2},
$$

the decay rates of the scalar boson eigenstate $H$ can then be obtained as

$$
\begin{aligned}
\Gamma(H \rightarrow h h) & =\frac{A_{H h h}^{2}}{8 \pi M_{H}}\left(1-4 \frac{m_{h}^{2}}{M_{H}^{2}}\right)^{1 / 2}, \\
\Gamma\left(H \rightarrow h_{1} h_{2}\right) & =\frac{A_{H h_{1} h_{2}}^{2}}{16 \pi M_{H}}\left(1-2 \frac{m_{h_{1}}^{2}+m_{h_{2}}^{2}}{M_{H}^{2}}+\frac{\left(m_{h_{1}}^{2}-m_{h_{2}}^{2}\right)^{2}}{M_{H}^{4}}\right)^{1 / 2} .
\end{aligned}
$$


Open Access. This article is distributed under the terms of the Creative Commons Attribution License (CC-BY 4.0), which permits any use, distribution and reproduction in any medium, provided the original author(s) and source are credited.

\section{References}

[1] Z. Chacko, H.-S. Goh and R. Harnik, The Twin Higgs: Natural electroweak breaking from mirror symmetry, Phys. Rev. Lett. 96 (2006) 231802 [hep-ph/0506256] [INSPIRE].

[2] G. Burdman, Z. Chacko, H.-S. Goh and R. Harnik, Folded supersymmetry and the LEP paradox, JHEP 02 (2007) 009 [hep-ph/0609152] [INSPIRE].

[3] P. Batra and Z. Chacko, A Composite Twin Higgs Model, Phys. Rev. D 79 (2009) 095012 [arXiv:0811.0394] [INSPIRE].

[4] M. Geller and O. Telem, Holographic Twin Higgs Model, Phys. Rev. Lett. 114 (2015) 191801 [arXiv: 1411.2974] [INSPIRE].

[5] R. Barbieri, D. Greco, R. Rattazzi and A. Wulzer, The Composite Twin Higgs scenario, JHEP 08 (2015) 161 [arXiv: 1501.07803] [INSPIRE].

[6] M. Low, A. Tesi and L.-T. Wang, Twin Higgs mechanism and a composite Higgs boson, Phys. Rev. D 91 (2015) 095012 [arXiv:1501.07890] [InSPIRE].

[7] N. Craig, S. Knapen and P. Longhi, The Orbifold Higgs, JHEP 03 (2015) 106 [arXiv: 1411.7393] [INSPIRE].

[8] N. Craig, S. Knapen and P. Longhi, Neutral Naturalness from Orbifold Higgs Models, Phys. Rev. Lett. 114 (2015) 061803 [arXiv:1410.6808] [INSPIRE].

[9] N. Craig, S. Knapen, P. Longhi and M. Strassler, The Vector-like Twin Higgs, JHEP 07 (2016) 002 [arXiv: 1601.07181] [InSPIRE].

[10] R. Rattazzi, Naturalness and Neutral Naturalness, https://indico.cern.ch/event/559310/ contributions/2257059/attachments/1330732/1999754/rattazzi-oxford.pdf.

[11] A. Birkedal, Z. Chacko and M.K. Gaillard, Little supersymmetry and the supersymmetric little hierarchy problem, JHEP 10 (2004) 036 [hep-ph/0404197] [INSPIRE].

[12] P.H. Chankowski, A. Falkowski, S. Pokorski and J. Wagner, Electroweak symmetry breaking in supersymmetric models with heavy scalar superpartners, Phys. Lett. B 598 (2004) 252 [hep-ph/0407242] [INSPIRE].

[13] Z. Berezhiani, P.H. Chankowski, A. Falkowski and S. Pokorski, Double protection of the Higgs potential in a supersymmetric little Higgs model, Phys. Rev. Lett. 96 (2006) 031801 [hep-ph/0509311] [INSPIRE].

[14] T.S. Roy and M. Schmaltz, Naturally heavy superpartners and a little Higgs, JHEP 01 (2006) 149 [hep-ph/0509357] [INSPIRE].

[15] C. Csáki, G. Marandella, Y. Shirman and A. Strumia, The Super-little Higgs, Phys. Rev. D 73 (2006) 035006 [hep-ph/0510294] [INSPIRE].

[16] B. Bellazzini, S. Pokorski, V.S. Rychkov and A. Varagnolo, Higgs doublet as a Goldstone boson in perturbative extensions of the Standard Model, JHEP 11 (2008) 027 [arXiv:0805.2107] [INSPIRE]. 
[17] B. Bellazzini, C. Csáki, A. Delgado and A. Weiler, SUSY without the Little Hierarchy, Phys. Rev. D 79 (2009) 095003 [arXiv:0902.0015] [INSPIRE].

[18] A. Falkowski, S. Pokorski and M. Schmaltz, Twin SUSY, Phys. Rev. D 74 (2006) 035003 [hep-ph/0604066] [INSPIRE].

[19] S. Chang, L.J. Hall and N. Weiner, A supersymmetric twin Higgs, Phys. Rev. D 75 (2007) 035009 [hep-ph/0604076] [INSPIRE].

[20] N. Craig and K. Howe, Doubling down on naturalness with a supersymmetric twin Higgs, JHEP 03 (2014) 140 [arXiv:1312.1341] [INSPIRE].

[21] R. Torre, Status of Composite Twin Higgs, http://indico.ictp.it/event/7627/session/76/contribution/472.

[22] H. Beauchesne, K. Earl and T. Grégoire, The spontaneous $\mathbb{Z}_{2}$ breaking Twin Higgs, JHEP 01 (2016) 130 [arXiv: 1510.06069] [INSPIRE].

[23] R. Harnik, K. Howe and J. Kearney, Tadpole-Induced Electroweak Symmetry Breaking and PNGB Higgs Models, arXiv:1603.03772 [INSPIRE].

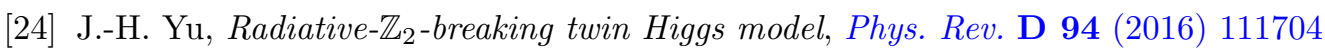
[arXiv: 1608.01314] [INSPIRE].

[25] J.-H. Yu, A tale of twin Higgs: natural twin two Higgs doublet models, JHEP 12 (2016) 143 [arXiv: 1608.05713] [INSPIRE].

[26] Z. Chacko, Y. Nomura, M. Papucci and G. Perez, Natural little hierarchy from a partially goldstone twin Higgs, JHEP 01 (2006) 126 [hep-ph/0510273] [INSPIRE].

[27] N. Craig, A. Katz, M. Strassler and R. Sundrum, Naturalness in the Dark at the LHC, JHEP 07 (2015) 105 [arXiv: 1501.05310] [INSPIRE].

[28] G. Burdman, Z. Chacko, R. Harnik, L. de Lima and C.B. Verhaaren, Colorless Top Partners, a $125 \mathrm{GeV}$ Higgs and the Limits on Naturalness, Phys. Rev. D 91 (2015) 055007 [arXiv:1411.3310] [INSPIRE].

[29] Z. Chacko, D. Curtin and C.B. Verhaaren, A Quirky Probe of Neutral Naturalness, Phys. Rev. D 94 (2016) 011504 [arXiv: 1512.05782] [InSPIRE].

[30] D. Curtin and C.B. Verhaaren, Discovering Uncolored Naturalness in Exotic Higgs Decays, JHEP 12 (2015) 072 [arXiv: 1506.06141] [INSPIRE].

[31] H.-C. Cheng, S. Jung, E. Salvioni and Y. Tsai, Exotic Quarks in Twin Higgs Models, JHEP 03 (2016) 074 [arXiv:1512.02647] [INSPIRE].

[32] N. Craig and A. Katz, The Fraternal WIMP Miracle, JCAP 10 (2015) 054 [arXiv: 1505.07113] [INSPIRE].

[33] I. García García, R. Lasenby and J. March-Russell, Twin Higgs Asymmetric Dark Matter, Phys. Rev. Lett. 115 (2015) 121801 [arXiv:1505.07410] [InSPIRE].

[34] I. García García, R. Lasenby and J. March-Russell, Twin Higgs WIMP Dark Matter, Phys. Rev. D 92 (2015) 055034 [arXiv: 1505. 07109] [INSPIRE].

[35] M. Farina, Asymmetric Twin Dark Matter, JCAP 11 (2015) 017 [arXiv:1506.03520] [INSPIRE].

[36] M. Farina, A. Monteux and C.S. Shin, Twin mechanism for baryon and dark matter asymmetries, Phys. Rev. D 94 (2016) 035017 [arXiv:1604.08211] [INSPIRE]. 
[37] R. Barbieri, L.J. Hall and K. Harigaya, Minimal Mirror Twin Higgs, JHEP 11 (2016) 172 [arXiv: 1609.05589] [INSPIRE].

[38] D. Buttazzo, F. Sala and A. Tesi, Singlet-like Higgs bosons at present and future colliders, JHEP 11 (2015) 158 [arXiv:1505.05488] [INSPIRE].

[39] D. Bertolini and M. McCullough, The Social Higgs, JHEP 12 (2012) 118 [arXiv:1207.4209] [INSPIRE].

[40] T. Robens and T. Stefaniak, Status of the Higgs Singlet Extension of the Standard Model after LHC Run 1, Eur. Phys. J. C 75 (2015) 104 [arXiv:1501.02234] [InSPIRE].

[41] A. Falkowski, C. Gross and O. Lebedev, A second Higgs from the Higgs portal, JHEP 05 (2015) 057 [arXiv: 1502.01361] [INSPIRE].

[42] M. Gorbahn, J.M. No and V. Sanz, Benchmarks for Higgs Effective Theory: Extended Higgs Sectors, JHEP 10 (2015) 036 [arXiv: 1502.07352] [InSPIRE].

[43] N. Craig, F. D'Eramo, P. Draper, S. Thomas and H. Zhang, The Hunt for the Rest of the Higgs Bosons, JHEP 06 (2015) 137 [arXiv: 1504.04630] [INSPIRE].

[44] A. Katz, M. Reece and A. Sajjad, Naturalness, $b \rightarrow s \gamma$ and SUSY heavy Higgses, JHEP 10 (2014) 102 [arXiv:1406.1172] [INSPIRE].

[45] R. Barbieri and G.F. Giudice, Upper Bounds on Supersymmetric Particle Masses, Nucl. Phys. B 306 (1988) 63 [INSPIRE].

[46] P. Batra, A. Delgado, D.E. Kaplan and T.M.P. Tait, The Higgs mass bound in gauge extensions of the minimal supersymmetric standard model, JHEP 02 (2004) 043 [hep-ph/0309149] [INSPIRE].

[47] S. Davidson, S. Hannestad and G. Raffelt, Updated bounds on millicharged particles, JHEP 05 (2000) 003 [hep-ph/0001179] [INSPIRE].

[48] A. Hook, E. Izaguirre and J.G. Wacker, Model Independent Bounds on Kinetic Mixing, Adv. High Energy Phys. 2011 (2011) 859762 [arXiv:1006.0973] [INSPIRE].

[49] CMS collaboration, Search for a Higgs boson in the mass range from 145 to $1000 \mathrm{GeV}$ decaying to a pair of $W$ or $Z$ bosons, JHEP 10 (2015) 144 [arXiv:1504.00936] [INSPIRE].

[50] CMS collaboration, Search for a heavy charged Higgs boson in proton-proton collisions at $\sqrt{s}=8 \mathrm{TeV}$ with the CMS detector, CMS-PAS-HIG-13-026.

[51] F. Gianotti et al., Physics potential and experimental challenges of the LHC luminosity upgrade, Eur. Phys. J. C 39 (2005) 293 [hep-ph/0204087] [INSPIRE].

[52] LHC Higgs Cross section Working Group collaboration, Handbook of LHC Higgs Cross sections: 3. Higgs Properties, arXiv:1307.1347 [INSPIRE].

[53] S. Dawson et al., Working Group Report: Higgs Boson, in proceedings of the Community Summer Study 2013: Snowmass on the Mississippi (CSS2013), Minneapolis, MN, U.S.A., July 29 - August 6, 2013, [arXiv:1310.8361] [INSPIRE].

[54] M. Misiak et al., Updated NNLO QCD predictions for the weak radiative B-meson decays, Phys. Rev. Lett. 114 (2015) 221801 [arXiv:1503.01789] [INSPIRE].

[55] K. Trabelsi, Rare decays and exotic states in quark flavour physics, in plenary talk at the EPS 2015 Conference, Vienna, Austria, July 22-29, 2015. 
[56] F. Staub, SARAH 4: A tool for (not only SUSY) model builders, Comput. Phys. Commun. 185 (2014) 1773 [arXiv: 1309.7223] [INSPIRE].

[57] D. Greco and K. Mimouni, The RG-improved Twin Higgs effective potential at NNLL, JHEP 11 (2016) 108 [arXiv:1609.05922] [INSPIRE].

[58] J. Hajer, Y.-Y. Li, T. Liu and J.F.H. Shiu, Heavy Higgs Bosons at $14 \mathrm{TeV}$ and $100 \mathrm{TeV}$, JHEP 11 (2015) 124 [arXiv: 1504.07617] [INSPIRE].

[59] S. Gori, I.-W. Kim, N.R. Shah and K.M. Zurek, Closing the Wedge: Search Strategies for Extended Higgs Sectors with Heavy Flavor Final States, Phys. Rev. D 93 (2016) 075038 [arXiv: 1602.02782] [INSPIRE].

[60] N. Craig, J. Hajer, Y.-Y. Li, T. Liu and H. Zhang, Heavy Higgs bosons at low $\tan \beta$ : from the LHC to $100 \mathrm{TeV}$, JHEP 01 (2017) 018 [arXiv:1605.08744] [INSPIRE].

[61] A. Coccaro, D. Curtin, H.J. Lubatti, H. Russell and J. Shelton, Data-driven Model-independent Searches for Long-lived Particles at the LHC, Phys. Rev. D 94 (2016) 113003 [arXiv: 1605.02742] [INSPIRE].

[62] J.P. Chou, D. Curtin and H.J. Lubatti, New Detectors to Explore the Lifetime Frontier, arXiv: 1606.06298 [INSPIRE]. 